\title{
DISTINGUISHING REMITTED BIPOLAR DISORDER FROM REMITTED \\ UNIPOLAR DEPRESSION IN PRE-ADOLESCENT CHILDREN: A NEURAL REWARD PROCESSING PERSPECTIVE
}

\author{
A Dissertation \\ Submitted to \\ The Temple University Graduate Board \\ In Partial Fulfillment \\ of the Requirements for the Degree \\ DOCTOR OF PHILOSOPHY \\ by \\ Ho-Yee $\mathrm{Ng}$ \\ August 2020 \\ Examining Committee Members: \\ Lauren B. Alloy, Ph.D., Advisory Chair, Department of Psychology \\ David V. Smith, Ph.D., Department of Psychology \\ Thomas M. Olino, Ph.D., Department of Psychology \\ Tania Giovannetti, Ph.D., Department of Psychology \\ Johanna Jarcho, Ph.D., Department of Psychology \\ Vishnu Murty, Ph.D., Department of Psychology
}


ABSTRACT

Bipolar disorder (BD) and unipolar depression (UD) are two severe mood disorders, with BD often misdiagnosed as UD. Given their severity and high rates of misdiagnosis, it is of paramount importance to understand the psychological and neurobiological mechanisms underlying these disorders to enhance our ability to diagnose, treat, and prevent them effectively.

Many neuroimaging studies have shown that mood disorders are associated with abnormal reward-related responses, particularly in the ventral striatum (VS). Yet, the link between mood disorders and reward-related responses in other regions remains inconclusive, thus limiting our understanding of the pathophysiology of mood disorders. To provide insights into the neurobiological underpinnings of reward processing dysfunction in mood disorders, two studies were conducted.

Study 1 (Chapter 2) is a coordinate-based meta-analysis of 41 whole-brain neuroimaging studies encompassing reward-related responses from a total of 794 patients with major depressive disorder (MDD), and 803 healthy controls (HC). It aims to address inconsistencies in the literature by synthesizing the literature quantitatively. The findings of Study 1 indicate that MDD is associated with opposing abnormalities in the reward circuit: hypo-responses in the VS and hyper-responses in the orbitofrontal cortex (OFC). These findings provide a foundation for Study 2 (Chapter 3) and help to reconceptualize our understanding of reward processing abnormalities in UD by suggesting a role for dysregulated corticostriatal connectivity.

Study 2 is the first fMRI study to employ region-of-interest (VS and OFC), whole-brain, activation, connectivity, and network analyses to examine the similarities and differences in reward-related brain activation patterns between 46 children with remitted bipolar I disorder, 48 children with remitted MDD, and 46 HC. 
The results of Study 2 revealed differential connectivity in corticostriatal circuitry during reward processing among $\mathrm{BD}, \mathrm{UD}$, and $\mathrm{HC}$ in pre-adolescence. Specifically, BD exhibited increases in OFC-VS connectivity during anticipation of larger reward, whereas UD and $\mathrm{HC}$ showed no changes in OFC-VS connectivity across anticipation conditions ranging from large loss to large reward. Furthermore, BD and UD generally showed more abnormal whole-brain responses to reward anticipation in accordance with the valence of the stimuli than HC. These findings suggest that preadolescents with BD and UD exhibit reward processing dysfunction during reward anticipation relative to $\mathrm{HC}$ even outside of acute periods of illness.

Taken together, the dissertation provides novel insight into the nature of reward processing abnormalities in mood disorders in pre-adolescence. As early onset $\mathrm{BD}$ or UD often is associated with long treatment delays and a persistently pernicious illness course, this dissertation may aid efforts to ensure early accurate diagnosis, which may improve our ability to intervene with appropriate treatments and result in a more benign prognosis and course of illness over the lifespan.

Keywords: bipolar disorder, major depressive disorder, reward processing, functional magnetic resonance imaging, pre-adolescence 


\section{DEDICATION}

For my advisor, Dr. Lauren Alloy, for her support and mentorship over the past five years. I am incredibly privileged to be under her wing and benefit from her brilliant mind and caring heart. I can only hope to someday be an ounce of the mentor and scientist she is.

For Dr. David Smith, who has patiently taught me how to conduct neuroimaging analyses and inspired me to be a better scientist. The completion of my dissertation would not have been possible without his support and nurturing.

For Drs. Thomas Olino, Tania Giovannetti, Johanna Jarcho, and Vishnu Murty, for the time and energy they have devoted as members of my dissertation committee.

For the Mood and Cognition Lab and my cohort, whose warmth and friendship have made the U.S. feel like home. In particular, many thanks to Madison Titone for being the best partner in crime one can ever ask for.

For my family, for allowing me to pursue my dream, even though that meant that I would move 8000 miles away and only be able to see them once every one or two years. 


\section{ACKNOWLEDGEMENTS}

I am indebted to the generous support I have received throughout my graduate school career: Sir Edward Youde Memorial Fellowship for Oversea Studies from the Sir Edward Youde Memorial Fund, Presidential Fellowship from Temple University, Elizabeth Munsterberg Koppitz Child Psychology Graduate Fellowship from the American Psychological Foundation (APF), Harry and Miriam Levinson Scholarship from the APF and the Council of Graduate Departments of Psychology (COGDOP), Psychological Science Research Grant from the American Psychological Association of Graduate Students (APAGS), and Computational Credits from the National Institute of Mental Health Data Archive (NDA). Their support has deepened my determination to make it my life's work to conduct meaningful research to improve the lives of individuals with or at risk for mental illnesses.

I would also like to express my deepest gratitude to the investigators and participants of the Adolescent Brain Cognitive Development (ABCD) Study (https://abcdstudy.org), whose data, held in the NDA, were used in this dissertation. This is a multisite, longitudinal study designed to recruit more than 10,000 children age 9-10 and follow them over 10 years into early adulthood. The ABCD Study is supported by the National Institutes of Health and additional federal partners under award numbers U01DA041022, U01DA041028, U01DA041048, U01DA041089, U01DA041106, U01DA041117, U01DA041120, U01DA041134, U01DA041148, U01DA041156, U01DA041174, U24DA041123, and U24DA041147. A full list of supporters is available at https://abcdstudy.org/nih-collaborators. A listing of participating sites and a complete listing of the study investigators can be found at https://abcdstudy.org/principal-investigators.html. ABCD consortium investigators designed and implemented the study and/or provided data but did not necessarily 
participate in analysis or writing of this report. This manuscript reflects the views of the authors and may not reflect the opinions or views of the NIH or ABCD consortium investigators. The $\mathrm{ABCD}$ data repository grows and changes over time. The ABCD data used in this report came from doi:10.15154/1412097. DOIs can be found at http://dx.doi.org/10.15154/1412097. 
TABLE OF CONTENTS

ABSTRACT FEDICATION Page

\section{CHAPTER}

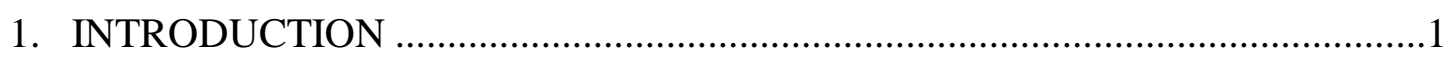

2. STUDY 1: A COORDINATE-BASED META-ANALYSIS OF NEUROIMAGING STUDIES ON REWARD PROCESSING DYSFUNCTION

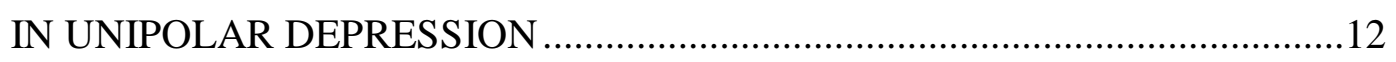

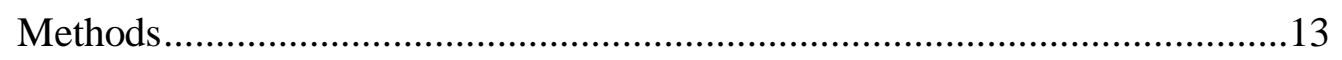

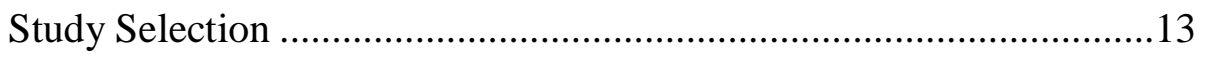

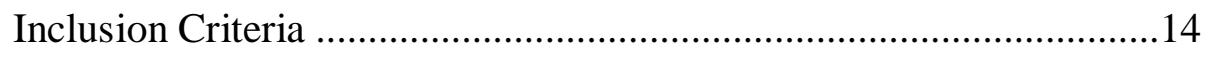

Coordinate-based Meta-analysis .......................................................15

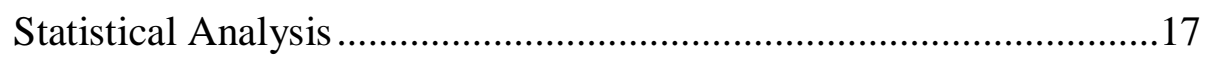

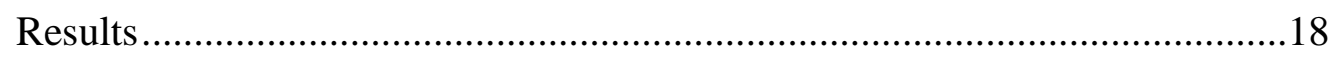

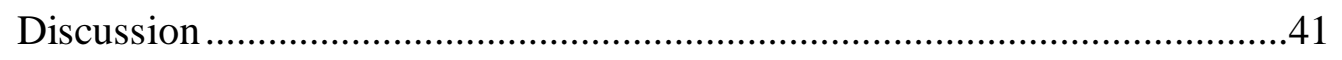

3. STUDY 2: AN FMRI STUDY OF REWARD PROCESSING DYSFUNCTION IN PREADOLESCENT MOOD DISORDERS ……………………….............49

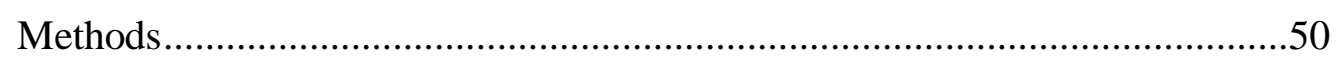

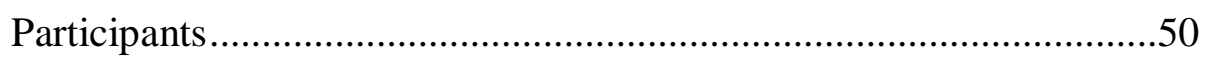

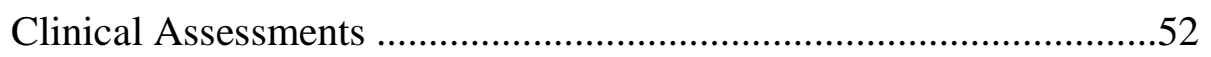




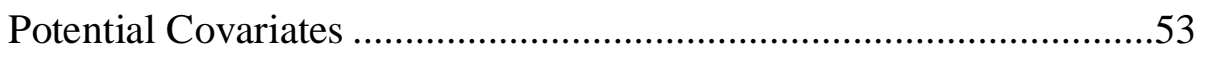

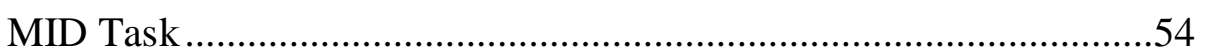

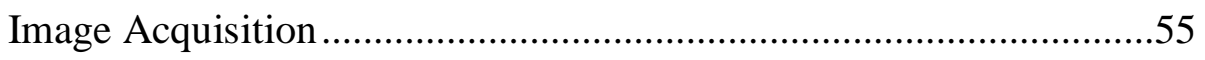

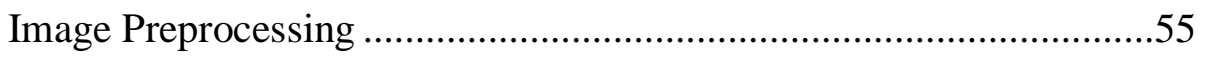

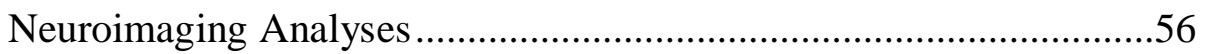

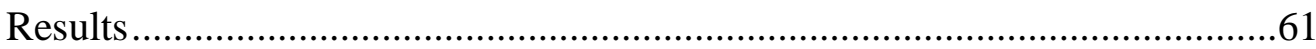

Participant Characteristics .........................................................61

Associations Among Study Variables ..............................................64

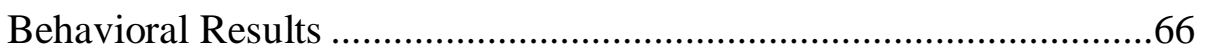

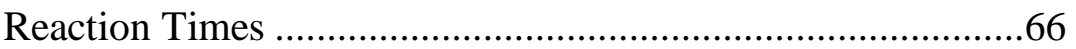

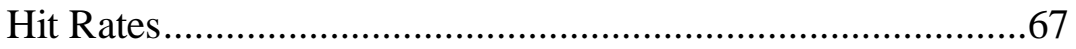

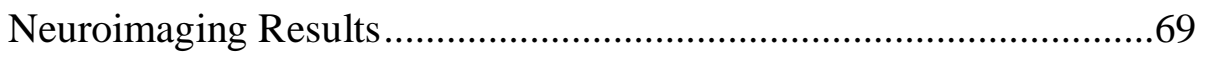

Activation Analyses .........................................................69

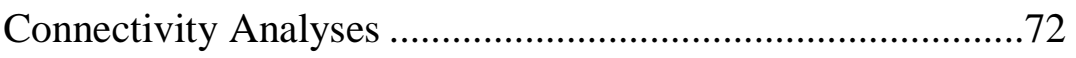

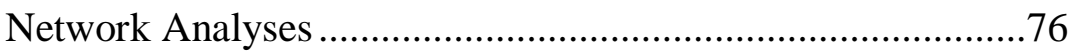

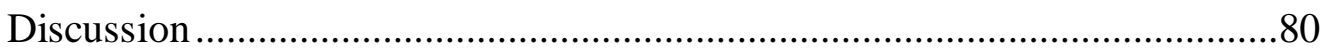

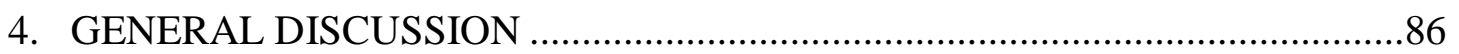

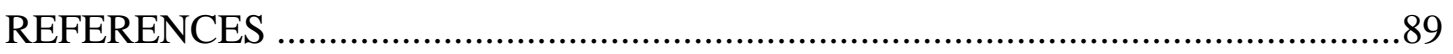




\section{LIST OF TABLES}

Table

1. Comparison of findings on reward responses (i.e., reward $>$ punishment/neutral)

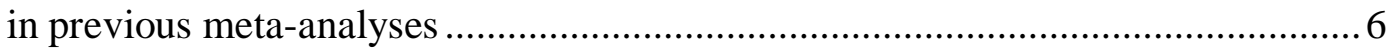

2. Characteristics of the study samples included in the meta-analysis .....................19

3. Characteristics of the studies included in the meta-analysis ...............................25

4. Peak coordinates of group differences in neural responses to reward ...................37

5. Peak coordinates of group differences in neural responses to reward (excluding

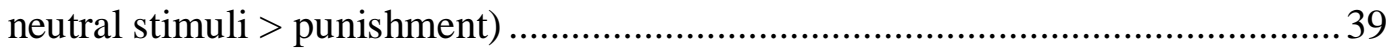

6. Peak coordinates of group differences in neural responses to punishment ..........40

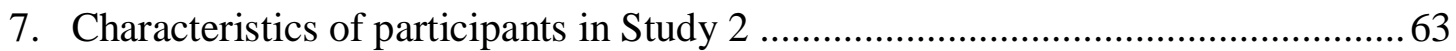

8. Pearson correlations between regions of interest and participant characteristics ..66 


\section{LIST OF FIGURES}

Figure

Page

1. Illustration of findings of previous meta-analyses on reward processing in unipolar depression 8

2. Flowchart of study selection 18

3. Opposing abnormalities in the reward circuit in response to reward in unipolar depression .38

4. Hyper-responses to punishment in the sublenticular extended amygdala in unipolar depression

5. CSNmaps derived from tensorial independent component analysis .58

6. Illustration of valence and salience signals based on study hypotheses 61

7. Reaction time by group and condition during the MID task

8. Hit rate by group and condition during the MID task. .68

9. Group differences in OFC activation during anticipation .70

10. Group differences in VS activation during anticipation .71

11. Group differences in OFC and VS activation during feedback .72

12. Group differences in OFC-VS functional connectivity .73

13. Group differences in OFC-VS connectivity during anticipation .75

14. Group differences in OFC-VS connectivity during feedback 76

15. Group differences in spatial correlations between the CSNmaps and the wholebrain responses during anticipation (valence)

16. Group differences in spatial correlations between the CSNmaps and the wholebrain responses during anticipation (salience)

17. Group differences in spatial correlations between the CSNmaps and the wholebrain responses during feedback 


\section{CHAPTER 1}

\section{INTRODUCTION}

Bipolar disorder (BD) and unipolar depression (UD) are two of the most debilitating conditions in the world. According to the World Health Organization (2006, 2017), both disorders are ranked among the top ten leading causes of disability and ill health worldwide. Moreover, it is estimated that BD and UD together afflict approximately one-fifth of the world's population during their lifetime (Bromet et al., 2011; Leonpacher et al., 2015; Merikangas et al., 2011). Given their severity and prevalence, it is of paramount importance to understand the neurobiological mechanisms underlying these disorders to enhance our ability to diagnose, treat, and prevent them.

Over the last few decades, there has been a large effort to discern the similarities and differences between $\mathrm{BD}$ and $\mathrm{UD}$, presumably because $\mathrm{BD}$ is often misdiagnosed as UD, especially on initial presentation (Angst et al., 2011; Ghaemi, Boiman, \& Goodwin, 2000; Ghaemi, Sachs, Chiou, Pandurangi, \& Goodwin, 1999; Leonpacher et al., 2015). The latency from onset of BD to receipt of an accurate diagnosis of BD can range from five to 15 years (Ghaemi et al., 1999; Hirschfeld, Lewis, \& Vornik, 2003; Pendergast et al., 2014). Prior research has shown that $69 \%$ of people with BD have been misdiagnosed in their lifetime (Lish, Dime-Meenan, Whybrow, Price, \& Hirschfeld, 1994), and that $60 \%$ of people with BD are initially diagnosed as having UD (Goodwin \& Jamison, 2007; Hirschfeld et al., 2003). Because some treatments for UD are contraindicated for $\mathrm{BD}$, misdiagnosis of $\mathrm{BD}$ as UD may lead to inappropriate treatment, resulting in poorer prognosis and illness course, such as increased risks of conversion to BD, rapid cycling, mood instability, and treatment resistance (Altshuler et al., 1995; Bowden, 2005; Leonpacher et al., 
2015; Perlis et al., 2010; Wehr \& Goodwin, 1987). Consequently, there is an urgent need to identify objective markers of BD and UD that might help differentiate the two debilitating conditions (Woo, Chang, Lindquist, \& Wager, 2017).

Growing interest in identifying objective markers of BD and UD has prompted many researchers to examine the ways in which reward processing goes awry in BD and UD, relative to healthy controls (HC). Evidence drawn from a variety of methods and measures suggests that both BD and UD exhibit reward processing abnormalities, relative to $\mathrm{HC}$, although their abnormalities appear to be on the opposite ends of the reward responsivity spectrum (Alloy, Nusslock, \& Boland, 2015; Alloy, Olino, Freed, \& Nusslock, 2016; Heshmati \& Russo, 2015; Nusslock \& Alloy, 2017; Russo \& Nestler, 2013; Whitton, Treadway, \& Pizzagalli, 2015). On the one hand, BD, especially during (hypo)mania, is generally characterized by reward hypersensitivity, as illustrated by neuroimaging research showing increased activity in the striatum during reward processing in BD, relative to HC (Alloy et al., 2015, 2016; Chase et al., 2013; Linke et al., 2012; Nusslock et al., 2012; Phillips \& Swartz, 2014; Singh et al., 2014; Whitton et al., 2015). On the other hand, UD is generally characterized by reward hyposensitivity, as demonstrated by neuroimaging research showing reduced activity in response to rewards in the striatum in UD, relative to $\mathrm{HC}$ (Bress, Foti, Kotov, Klein, \& Hajcak, 2013; Gotlib et al., 2010; Hall, Milne, \& MacQueen, 2014; Morgan, Olino, McMakin, Ryan, \& Forbes, 2013; Ng, Alloy, \& Smith, 2018; Olino et al., 2014; Pizzagalli et al., 2009; Robbins, 2016; Whitton et al., 2015).

The striatum, which can be divided into dorsal and ventral sections, is the primary input zone for basal ganglia (Haber, 2016; Haber \& Knutson, 2010). It receives afferent projections from the midbrain, amygdala, and prefrontal cortex (PFC), such as the orbitofrontal cortex (OFC), dorsolateral prefrontal cortex (dlPFC), 
ventromedial prefrontal cortex (vmPFC), and anterior cingulate cortex (ACC; Haber, 2016; Haber \& Knutson, 2010). It also projects to such regions as the ventral pallidum, ventral tegmental area, and substantia nigra (Haber \& Knutson, 2010). Many of the regions linked to the striatum, particularly prefrontal regions, have been associated with the computation and representation of reward value (Berridge $\&$ Kringelbach, 2015; Der-Avakian \& Markou, 2012; Kringelbach, 2005; Levy \& Glimcher, 2012; Padoa-Schioppa, 2011; Padoa-Schioppa \& Conen, 2017; Rangel, Camerer, \& Montague, 2008; Saez, Saez, Paton, Lau, \& Salzman, 2017; Stalnaker, Cooch, \& Schoenbaum, 2015; K. S. Wang, Smith, \& Delgado, 2016), as well as the regulation of affect and reward-related behavior in animals and healthy individuals (Delgado et al., 2016; Ferenczi et al., 2016; Peters \& Büchel, 2010; Phelps, Lempert, \& Sokol-Hessner, 2014; Voorn, Vanderschuren, Groenewegen, Robbins, \& Pennartz, 2004). The striatum also has been proposed to play an important role in the onset and course of mood psychopathology, with longitudinal studies demonstrating that blunted activation in the ventral striatum (VS) during reward anticipation predicts the emergence of depressive symptoms and disorder (Morgan et al., 2013; Stringaris et al., 2015) and deep-brain stimulation studies using it as a treatment target for treatment-resistant depression (Naesström, Blomstedt, \& Bodlund, 2016).

Although abnormal striatal response to reward in mood disorders is a relatively consistent finding in the literature (Alloy et al., 2016; Groenewold, Opmeer, de Jonge, Aleman, \& Costafreda, 2013; Hanson, Hariri, \& Williamson, 2015; Heshmati \& Russo, 2015; Nusslock \& Alloy, 2017; Nusslock et al., 2012; Whitton et al., 2015; W. N. Zhang, Chang, Guo, Zhang, \& Wang, 2013), it is less clear how other regions, particularly the $\mathrm{PFC}$, may also contribute to reward processing deficits in mood disorders. For instance, some studies have found that relative to HC, individuals 
with BD or UD exhibited greater activation in the OFC (Forbes et al., 2006; Nusslock et al., 2012; Smoski et al., 2009), dlPFC (Demenescu et al., 2011; Forbes et al., 2009; Pizzagalli et al., 2009), vmPFC (Keedwell, Andrew, Williams, Brammer, \& Phillips, 2005; Rizvi et al., 2013), ACC (Dichter, Kozink, McClernon, \& Smoski, 2012; Mitterschiffthaler et al., 2003), middle frontal gyrus (Dichter et al., 2012; Keedwell et al., 2005), inferior frontal gyrus (Kumari et al., 2003; Mitterschiffthaler et al., 2003), subgenual cingulate (Kumari et al., 2003; Rizvi et al., 2013), and dorsomedial prefrontal cortex (Keedwell et al., 2005) during the processing of rewarding stimuli. In contrast, other studies have reported less activity in BD or UD in response to reward in the OFC (Dichter et al., 2012; Forbes et al., 2006; Redlich et al., 2015), ACC (Forbes et al., 2006; Kumari et al., 2003; Pizzagalli et al., 2009; Smoski et al., 2009), middle frontal gyrus (Kumari et al., 2003; Mitterschiffthaler et al., 2003; Smoski et al., 2009), and frontal pole (Dichter et al., 2012). The inconsistencies may be due to a number of factors, such as limited statistical power (Button et al., 2013; Jia et al., 2018; Poldrack et al., 2017) and susceptibility artifacts in the ventral portions of the PFC (Andersson, Hutton, Ashburner, Turner, \& Friston, 2001; Chase, Kumar, Eickhoff, \& Dombrovski, 2015; Delgado et al., 2016; Ojemann et al., 1997). Therefore, the association between prefrontal regions and mood disorders remains equivocal, both in terms of the direction (i.e., hyper- or hypo-responses) and the location (e.g., OFC, dlPFC, vmPFC and/or ACC) of the effect.

Inconsistencies in the literature have prompted researchers to conduct coordinate-based meta-analyses to identify common activation patterns implicated in UD during reward processing. Although prior meta-analytic efforts (Groenewold et al., 2013; Keren et al., 2018; W. N. Zhang et al., 2013) have shown some overlapping findings in the striatum in UD, there is a striking degree of anatomical disagreement 
across these efforts, with non-overlapping findings all throughout the brain (see Table 1 and Figure 1 for a complete comparison of findings across meta-analyses). The lack of agreement across meta-analyses can be due to methodological issues, such as lenient thresholding, overlapping samples, software issues (Eickhoff, Laird, Fox, Lancaster, \& Fox, 2017), and inclusion of region-of-interest (ROI) coordinates, as detailed in a previous review (Müller, Cieslik, Serbanescu, et al., 2017). For example, two previous meta-analyses (Groenewold et al., 2013; W. N. Zhang et al., 2013) corrected for multiple comparisons using the false discovery rate (FDR) approach, which has been shown to be inadequate in controlling the false positives among clusters in neuroimaging meta-analyses (Chumbley \& Friston, 2009; Eickhoff, Bzdok, Laird, Kurth, \& Fox, 2012) and might have contributed to the lack of agreement across studies. Last by not least, it is important to note that whereas a few coordinatebased meta-analyses of neuroimaging studies on reward processing dysfunction in UD have been conducted, none have been published in BD. 
Table 1

Comparison of Findings on Reward Responses (i.e., Reward > Punishment/Neutral)

in Previous Meta-analyses

\begin{tabular}{|c|c|c|c|}
\hline \multirow[t]{2}{*}{ Brain Region } & \multicolumn{3}{|c|}{ MNI Coordinates } \\
\hline & $\mathrm{x}$ & $\mathrm{y}$ & $\mathrm{z}$ \\
\hline \multicolumn{4}{|l|}{ Groenewold et al. (2013) } \\
\hline \multicolumn{4}{|l|}{$U D>H C$} \\
\hline Lingual Gyrus & 26 & -92 & -14 \\
\hline Olfactorius Cortex & 4 & 22 & -14 \\
\hline Middle Orbitofrontal & 2 & 26 & -14 \\
\hline Rectus & 2 & 30 & -24 \\
\hline Middle Orbitofrontal & 0 & 26 & -12 \\
\hline Rectus & 0 & 24 & -24 \\
\hline \multicolumn{4}{|l|}{$H C>U D$} \\
\hline Cerebellum & -16 & -74 & -28 \\
\hline Lingual Gyrus & -18 & -62 & -6 \\
\hline Fusiform Gyrus & -22 & -74 & -14 \\
\hline Inferior Occipital Gyrus & -30 & -80 & -12 \\
\hline Rolandic Operculum & -40 & -24 & 20 \\
\hline Insula & -36 & -24 & 22 \\
\hline Superior Temporal Gyrus & -40 & -36 & 12 \\
\hline Heschl Gyrus & -46 & -16 & 12 \\
\hline Postcentral Gyrus & -50 & -18 & 18 \\
\hline Supramarginal Gyrus & -50 & -22 & 18 \\
\hline Anterior Cingulate Cortex & -2 & 28 & 16 \\
\hline Anterior Cingulate Cortex & 4 & 32 & 14 \\
\hline Lingual Gyrus & -18 & -62 & -6 \\
\hline Cerebellum & -6 & -58 & -4 \\
\hline Calcarine Sulcus & -20 & -54 & 4 \\
\hline Fusiform Gyrus & -26 & -58 & -12 \\
\hline Precuneus & -20 & -52 & 2 \\
\hline Pallidum & 18 & 0 & -4 \\
\hline Putamen & 28 & -4 & 8 \\
\hline Thalamus & 14 & -8 & 0 \\
\hline Insula & 38 & 10 & -12 \\
\hline Amygdala & 30 & -2 & -12 \\
\hline Caudate & 16 & 26 & 6 \\
\hline Fusiform & 44 & -62 & -20 \\
\hline Crus Cerebellum & 44 & -64 & -20 \\
\hline \multirow[t]{2}{*}{ Brain Region } & \multicolumn{3}{|c|}{ TAL Coordinates } \\
\hline & $\mathrm{x}$ & $\mathrm{y}$ & $\mathrm{Z}$ \\
\hline \multicolumn{4}{|l|}{ Zhang et al. (2013) } \\
\hline \multicolumn{4}{|l|}{$U D>H C$} \\
\hline Cuneus & 4 & -86 & 18 \\
\hline Cuneus & -6 & -86 & 22 \\
\hline
\end{tabular}


Table 1, continued

$\begin{array}{llll}\text { Frontal Lobe } & 20 & 30 & -6 \\ \text { Middle Frontal Gyrus } & 40 & 28 & 38 \\ \text { Superior Frontal Gyrus } & -4 & 48 & 32 \\ \text { Fusiform Gyrus } & -48 & -74 & -12 \\ \text { Middle Frontal Gyrus } & -48 & 14 & 30 \\ \text { Lingual Gyrus } & 12 & -52 & 4 \\ \text { Lingual Gyrus } & 14 & -54 & 0\end{array}$

$H C>U D$

Caudate

Caudate

Thalamus

$\begin{array}{lll}-6 & 18 & 4\end{array}$

$\begin{array}{lll}-8 & -8 & 10\end{array}$

Thalamus

$-10$

$-12 \quad 8$

Caudate

$-14$

$-14 \quad 16$

Cerebellum

$-12$

$-4 \quad 20$

Cerebellum

$4-36$

$-4$

Putamen

$-4 \quad-42$

$14 \quad 8$

Caudate

$14 \quad 14$

4

82

Anterior Cingulate

$-8 \quad 30$

10

Insula

34

Cerebellum

$-6$

$-4$

10

16

Brain Region

$-60$

$-20$

Coordinates

$\mathrm{x}$

Keren et al. (2018)

$H C>U D$

Caudate Body

Caudate Head

12

14

14

Caudate Body

6

2

$-2$

Note. MNI, Montreal Neurological Institute space; UD, unipolar depression; HC,

healthy controls; TAL, Talairach space. 


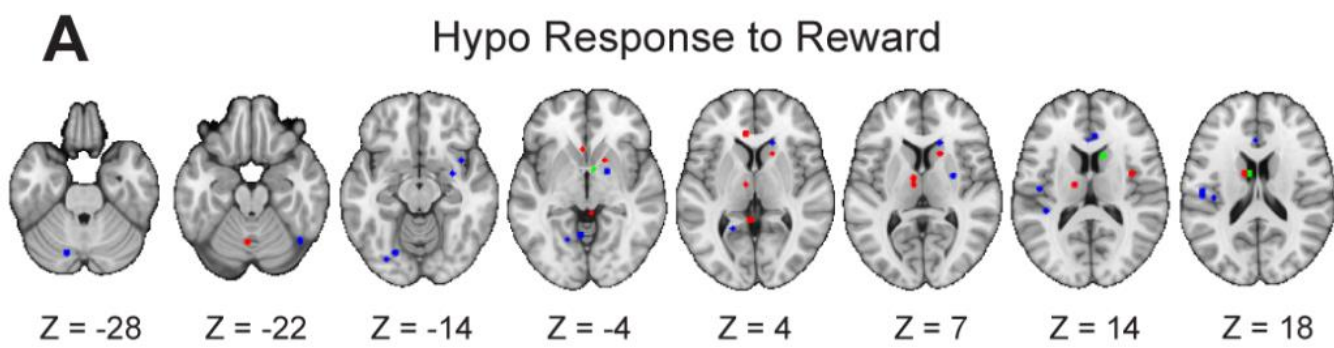

B

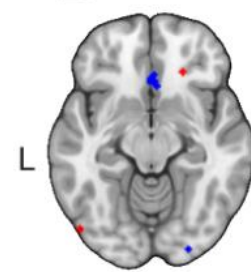

$\mathrm{Z}=-12$

Hyper Response to Reward

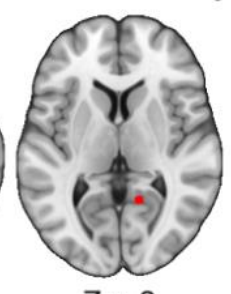

$\mathrm{Z}=6$

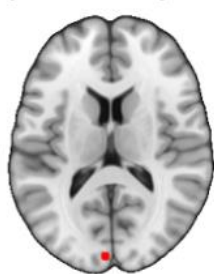

$Z=14$

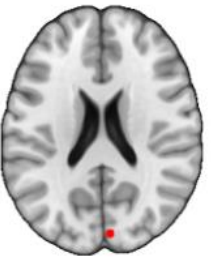

$Z=21$

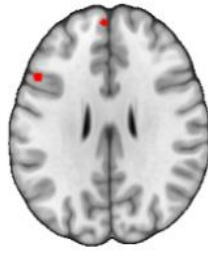

$Z=28$

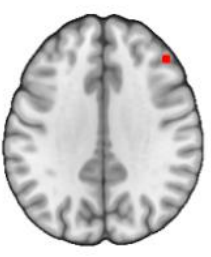

$Z=34$

Figure 1. Illustration of findings of previous meta-analyses on reward processing in unipolar depression. There is a striking degree of anatomical disagreement across these meta-analyses, with non-overlapping findings all throughout the brain. Blue represents Groenewold et al. (2013). Green represents Keren et al. (2018). Red represents Zhang et al. (2013). (A) Previous meta-analyses examining convergence among studies reporting hypo-responses to reward include Groenewold et al. (2013), Keren et al. (2018), and Zhang et al. (2013). (B) Previous meta-analyses examining convergence among studies reporting hyper-responses to reward include Groenewold et al. (2013) and Zhang et al. (2013).

Although a host of studies have observed reward processing abnormalities in BD or UD separately, only four studies have adopted functional magnetic resonance imaging (fMRI) to directly compare individuals with BD and UD during the performance of a reward task. Whereas one study demonstrated that adults with bipolar I depression had greater activity in the vlPFC during anticipation of monetary reward than adults with UD (Chase et al., 2013), another study found that adults with bipolar I depression had less activity in the striatum, thalamus, insula, and PFC than 
adults with UD during receipt of monetary reward (Redlich et al., 2015). Additionally, two other studies did not find any significant differences in brain activity associated with receipt of monetary or social reward between adults with bipolar I or II depression and adults with UD (Satterthwaite et al., 2015; Sharma et al., 2016). All four studies used a card-guessing paradigm (Delgado, Nystrom, Fissell, Noll, \& Fiez, 2000; Forbes et al., 2009; Holm et al., 2009; Nusslock et al., 2012; Wolf et al., 2014). Taken together, the literature comparing BD and UD on reward processing is scarce and mixed, with evidence indicating that adults with BD exhibit increased, decreased, or the same activation patterns during reward processing, relative to their counterparts with UD.

Another area of research that deserves more attention is reward functioning in pre-adolescent children with mood disorders. Examination of reward functioning in pre-adolescents with mood disorders is of particular interest, for at least three reasons. First, accumulating evidence suggests that reward sensitivity changes across the lifespan, increasing from pre-adolescence to late adolescence and decreasing thereafter (Casey, Jones, \& Hare, 2008; Chein, Albert, O’Brien, Uckert, \& Steinberg, 2011; Eppinger, Nystrom, \& Cohen, 2012; Green, Fry, \& Myerson, 1994; Somerville, Jones, \& Casey, 2010; Steinberg, 2007, 2008, 2010; Steinberg et al., 2008, 2009, 2018; Urošević, Collins, Muetzel, Lim, \& Luciana, 2012). These developmental changes appear to be coupled with neurobiological and structural alterations in the reward circuitry (Luciana \& Collins, 2012; Urošević et al., 2012). In view of the changes in reward functioning from pre-adolescence to adulthood, findings established in the literature on reward processing dysfunction in adults or adolescents with mood disorders may not be generalizable to pre-adolescents with mood disorders. Second, early onset BD or UD is often associated with remarkably long 
treatment delays and a persistently pernicious course of illness, underscoring the significance of studying mood disorders during this developmental period (Post et al., 2010; Zisook et al., 2007). Third, owing to the paucity of research on pre-adolescent mood disorders, diagnosing mood disorders in this population accurately is challenging (Beljan et al., 2006; Bhargava Raman et al., 2007; Carlson, 2000; Chang, 2009; Chilakamarri, Filkowski, \& Ghaemi, 2011; Geller \& Luby, 1997; Kessler, Avenevoli, \& Ries Merikangas, 2001; Luby, 2013; Rutter, 2011; Weller, Weller, \& Fristad, 1995; Youngstrom, Birmaher, \& Findling, 2008). Identifying objective markers of pre-adolescent BD and UD has the potential to enhance our ability to diagnose pediatric mood disorders accurately. Besides, early accurate diagnosis may improve our ability to intervene with appropriate treatments that may lead to a more benign course of mood disorder in adolescence and adulthood.

Taken together, further research is needed to better understand the neurobiological mechanisms underlying BD and UD in different stages of the lifespan to enhance our ability to diagnose, treat, and prevent them. To address this need, I conducted two studies. Study 1 (Chapter 2) is a coordinate-based meta-analysis of 41 whole-brain neuroimaging studies encompassing reward-related responses from a total of 794 patients with UD and 803 HC. It aims to summarize the current literature quantitively and provide a theoretical foundation for Study 2 (Chapter 3). Study 2 is an fMRI study that employs ROI, whole-brain, connectivity, and network analyses to examine the similarities and differences in reward-related brain activation patterns between 46 children with remitted BD, 48 children with remitted UD, and $46 \mathrm{HC}$. It provides the first examination of reward processing dysfunction in pre-adolescents with BD or UD using fMRI. In theoretical terms, Study 2 aims to fill in crucial knowledge gaps about the reward sensitivity theories of mood disorders by helping to 
clarify whether such dysfunction begins as early as pre-adolescence and whether reward processing abnormalities are stable characteristics of BD or UD, occurring even in the remitted state. In applied terms, the study aims to facilitate current endeavors to identify objective markers of BD and UD by informing understanding of neural circuitry of reward processing implicated in BD and UD. 


\section{CHAPTER 2}

\section{STUDY 1: A COORDINATE-BASED META-ANALYSIS OF NEUROIMAGING STUDIES ON REWARD PROCESSING DYSFUNCTION IN UNIPOLAR} DEPRESSION

Given the inconsistencies in the findings of published coordinate-based metaanalyses of neuroimaging studies on reward processing dysfunction in UD and the lack of such work in BD, the initial goal of Study 1 was to provide a methodologically rigorous synthesis of the literature on reward processing dysfunction in both BD and UD. Nevertheless, the current meta-analysis only focused on UD because of an inadequate number of studies on $\mathrm{BD}$, as the systematic literature search yielded fewer than $17 \mathrm{BD}$ experiments for each targeted meta-analysis and 17 experiments is the minimum number needed to minimize bias and restrict excessive contribution of any particular studies to cluster-level thresholding in coordinate-based meta-analysis (Eickhoff et al., 2016).

The current meta-analysis followed procedures recommended by new guidelines (Müller, Cieslik, Laird, et al., 2017; Müller, Cieslik, Serbanescu, et al., 2017). As a result, the current work differed from previous meta-analyses on reward processing in UD in various aspects, such as only including whole-brain studies to avoid localization bias; only including studies that used an active control condition to isolate reward-related processes; only including independent samples to avoid double counting the same participants; using more stringent thresholding criteria; having the most up-to-date literature search; and only conducting a meta-analysis when there were at least 17 eligible experiments.

The primary hypothesis of Study 1 was that the literature would consistently show that compared with HC, individuals with UD would exhibit blunted activation 
of the striatum and abnormal activation of the prefrontal regions (e.g., the OFC) during the processing of rewarding stimuli. I also explored whether there were consistent neural responses to punishing stimuli in UD relative to $\mathrm{HC}$. To examine these questions, I conducted four separate coordinate-based meta-analyses testing spatial convergence of neuroimaging findings for the following four contrasts: 1) positive valence (reward > punishment/neutral stimuli or neutral stimuli > punishment) for UD > HC; 2) negative valence (punishment > reward/neutral stimuli or neutral stimuli > reward) for UD > HC; 3) positive valence for $\mathrm{HC}>\mathrm{UD}$; and 4) negative valence for HC > UD. Neutral stimuli were considered rewarding relative to punishing stimuli but punishing relative to rewarding stimuli, in view of the idea that brain activation during reward processing reflects the valence of a stimulus and follows a linear pattern (Bartra, McGuire, \& Kable, 2013; Cooper \& Knutson, 2008). The comprehensive and rigorous nature of the current meta-analysis allowed for the investigation of whether a quantitative synthesis of neuroimaging studies on reward-related processing in UD would unveil common activation patterns that may be difficult to discern by individual studies due to inconsistent findings. I aimed to address two main questions. First, which brain regions show consistent hyporesponses to reward-relevant stimuli in UD relative to HC? Second, which brain regions show consistent hyper-responses to reward-relevant stimuli in UD relative to $\mathrm{HC}$ ?

\section{Methods}

\section{Study Selection}

The current coordinate-based meta-analysis primarily followed the guidelines for meta-analyses, whenever applicable (Moher, Liberati, Tetzlaff, Altman, \& PRISMA Group, 2009; Müller, Cieslik, Laird, et al., 2017). I conducted a systematic 
literature search to identify neuroimaging studies on reward processing abnormalities in mood disorders. Potentially eligible studies published between 1/1/1997 and 8/7/2018 were identified by searching the MEDLINE, EMBASE, PsycINFO, PsycARTICLES, Scopus, and Web of Science using the grouped terms (fMRI* or PET*) AND (depress* OR bipolar* OR mania* OR manic* OR hypomania* OR hypomanic*) AND (reward* OR effort* OR decision* OR reinforce* OR habit* OR discounting* OR "prediction error” OR “delayed gratification” OR “approach motivation" OR "positive valence systems"). To enhance search sensitivity, the reference lists of the retrieved articles and review papers were further checked to identify potentially relevant articles.

\section{Inclusion Criteria}

I included studies that (a) used a reward and/or punishment task, (b) reported comparisons between people with UD and HC, (c) used standardized diagnostic criteria (e.g., DSM-IV, DSM-IV-TR, ICD-10) to determine psychiatric diagnoses, (d) used fMRI or PET in conjunction with parametric analysis or subtraction methodology contrasting an experimental condition and an active control condition (e.g., a punishment condition, a lower-intensity reward condition, or a neutral condition) to isolate reward-related processes and identify foci of task-related neural changes, (e) reported significant results of whole-brain group analyses without small volume corrections (SVC), as non-whole-brain coordinates [e.g., ROI-based coordinates] and analyses involving SVC have been argued to bias coordinate-based meta-analyses (Eickhoff et al., 2016; Müller, Cieslik, Laird, et al., 2017), (f) reported coordinates in a standard stereotactic space [Talairach or Montreal Neurological Institute (MNI) space], and (g) used independent samples. Authors were contacted when information were unavailable in the published reports. 
The study with the largest sample size was included if there was sample overlap between studies. Reward tasks were operationalized as involving presentation of a rewarding stimulus (e.g., winning money, favorite music, positive faces), whereas punishment tasks were operationalized as involving presentation of a punishing stimulus (e.g., losing money, negative faces). The stimuli used in the included studies of the meta-analysis reflect a reward-punishment or positive-negative continuum. For example, positive faces are considered as rewards based on previous research showing that positive faces activate the reward circuitry, that they are discounted as a function of time, that they are tradable for other rewards (e.g., money), that they reinforce work, and that people are willing to work to view positive faces and exert more effort for more positive faces (Hayden, Parikh, Deaner, \& Platt, 2007; Tsukiura \& Cabeza, 2008).

\section{Coordinate-Based Meta-Analysis}

Coordinate-based meta-analyses were performed using GingerALE 2.3.6 (http:// brainmap.org), which employs the activation likelihood estimation (ALE) method (Eickhoff et al., 2012; Turkeltaub et al., 2012). The ALE method aims to identify regions showing spatial convergence between experiments and tests against the null hypothesis that the foci of experiments are uniformly and randomly distributed across the brain (Eickhoff et al., 2012). It treats foci from individual experiments as centers for 3D Gaussian probability distributions representing spatial uncertainty. The width of these distributions was determined based on betweensubject and between-template variability (Eickhoff et al., 2009). The ALE algorithm weighs the between-subject variability by the number of participants for each study, based on the idea that experiments of larger sample sizes are more likely to reliably report true activation effects. Therefore, experiments with larger sample sizes are 
modeled by smaller Gaussian distributions, resulting in a stronger influence on ALE scores, which indicate the probability that at least one true peak activation lies in the voxel across the population of all possible studies (Eickhoff et al., 2009). As a result, studies with larger sample sizes would be weighed more heavily relative to studies with smaller sample sizes.

The ALE method is implemented in the following steps. First, for each included study, a map of the activation likelihood is computed. Second, the maps are aggregated to compute the ALE score for each voxel. Finally, a permutation test is employed to identify voxels in which the ALE statistic is larger than expected by chance (Eickhoff et al., 2012, 2009; Turkeltaub et al., 2012). The ALE method takes into account heterogeneity in spatial uncertainty across studies (Eickhoff et al., 2012, 2009; Turkeltaub et al., 2012) and differences in number of peak coordinates reported per cluster (Turkeltaub et al., 2012). This approach allows random-effects estimates of ALE, increasing generalizability of the results (Eickhoff et al., 2009).

Coordinate-based meta-analyses represent a departure from traditional metaanalyses (Müller, Cieslik, Laird, et al., 2017). Specifically, whereas traditional metaanalyses aim to calculate pooled effect sizes to determine the direction and magnitude of an effect based on a body of literature, coordinate-based meta-analyses evaluate whether the location of an effect is consistent within a body of literature. In other words, coordinate-based meta-analyses are blind to effect size magnitude, but direction is tied to the analysis (Müller, Cieslik, Laird, et al., 2017). Although the coordinate-based meta-analytic approach is limited to studies that report suprathreshold coordinates for a given contrast, this approach will continue to be the most common method for conducting valid meta-analyses for neuroimaging studies until it becomes standard practice to release unthresholded statistical maps. 


\section{Statistical Analysis}

All analyses were performed in Montreal Neurological Institute (MNI) space. Coordinates reported in Talairach space were converted to MNI using the "icbm2tal" transformation (Lancaster et al., 2007). I assessed statistical significance and corrected for multiple comparisons using the permutation-based approach $(\mathrm{N}=1000)$ recommended by the developers of GingerALE (Eickhoff et al., 2017, 2016). This approach utilized a cluster-forming threshold of $p<0.001$ (uncorrected) and maintained a cluster-level family-wise error rate of 5\% (Eickhoff et al., 2016). Additionally, I took steps to minimize within-group effects on the meta-analyses (Turkeltaub et al., 2012). If a study reported more than one contrast (often referred to as an "experiment" in coordinate-based meta-analyses), the contrasts examining similar processes were pooled together to avoid double counting the same participants in a meta-analysis. For example, when a study reported between-group effects in response to $\$ 1.50$ and $\$ 5$ rewards relative to neutral or loss conditions, the coordinates derived from the two contrasts were coded as a single reward experiment.

To capture anatomical variation between individual human brains (Mazziotta, Toga, Evans, Fox, \& Lancaster, 1995), I show probabilistic anatomical labels for the locations of the maximum ALE values using the Harvard-Oxford cortical and subcortical atlases (Desikan et al., 2006). For transparency, all of the statistical maps (thresholded and unthresholded) derived from the meta-analyses are publicly available on NeuroVault (https://neurovault.org/collections/3884/). Readers are free to access these maps and define these regions using their own labels. Study materials are available on Open Science Framework at https://osf.io/sjb4d. 


\section{Results}

As shown in Figure 2, the systematic literature search identified a total of 41 neuroimaging studies that met the inclusion criteria, yielding 4 coordinate-based meta-analyses with at least 17 independent experiments. Tables 2 and 3 show the characteristics of the included studies and their samples.

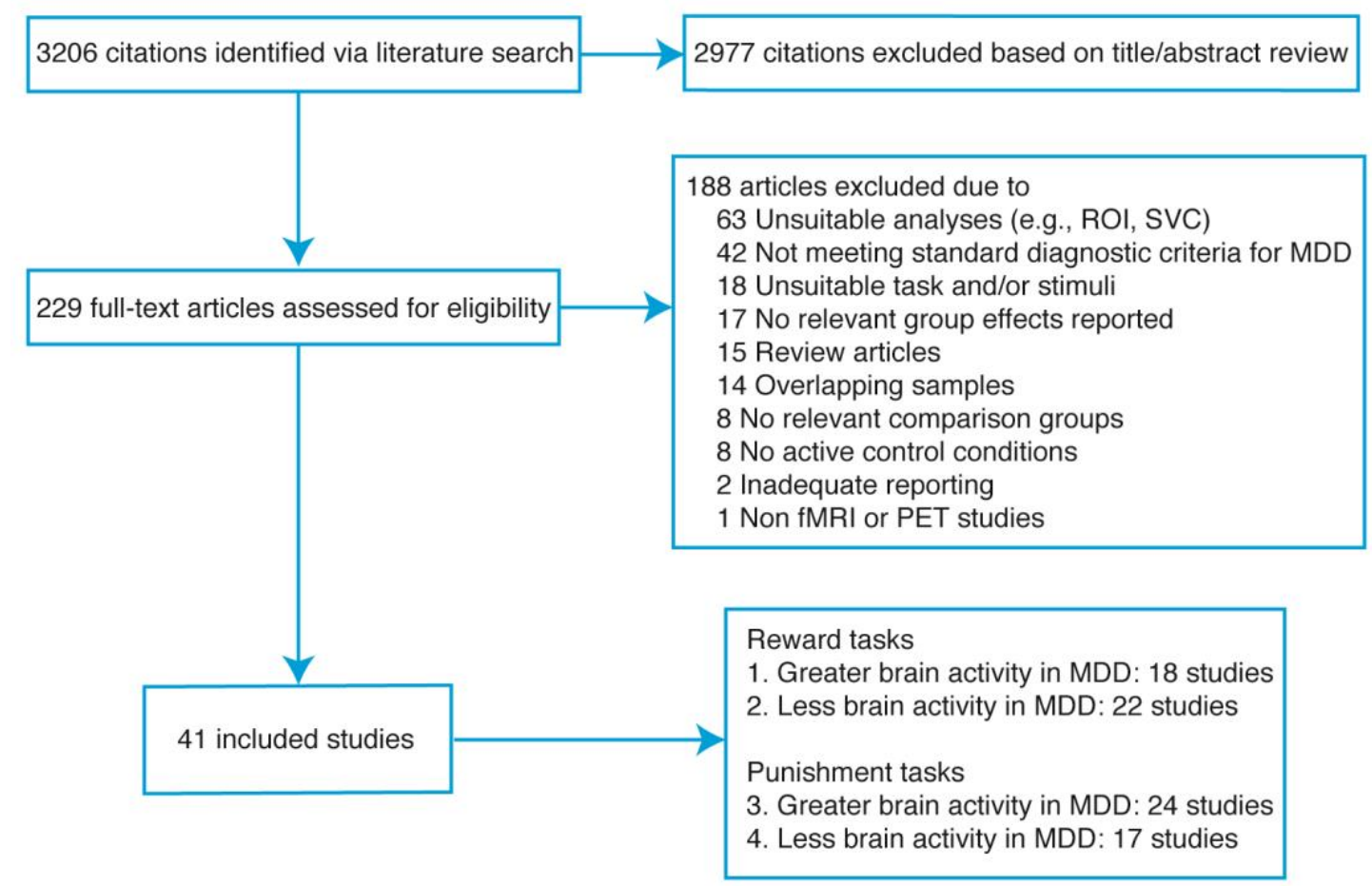

Figure 2. Flowchart of study selection. The systematic literature search identified a total of 41 neuroimaging studies that met the inclusion criteria, yielding 4 coordinatebased meta-analyses with at least 17 independent studies; ROI, region of interest; SVC, small volume correction; MDD, major depressive disorder. 
Table 2

Characteristics of the Study Samples Included in the Meta-analysis

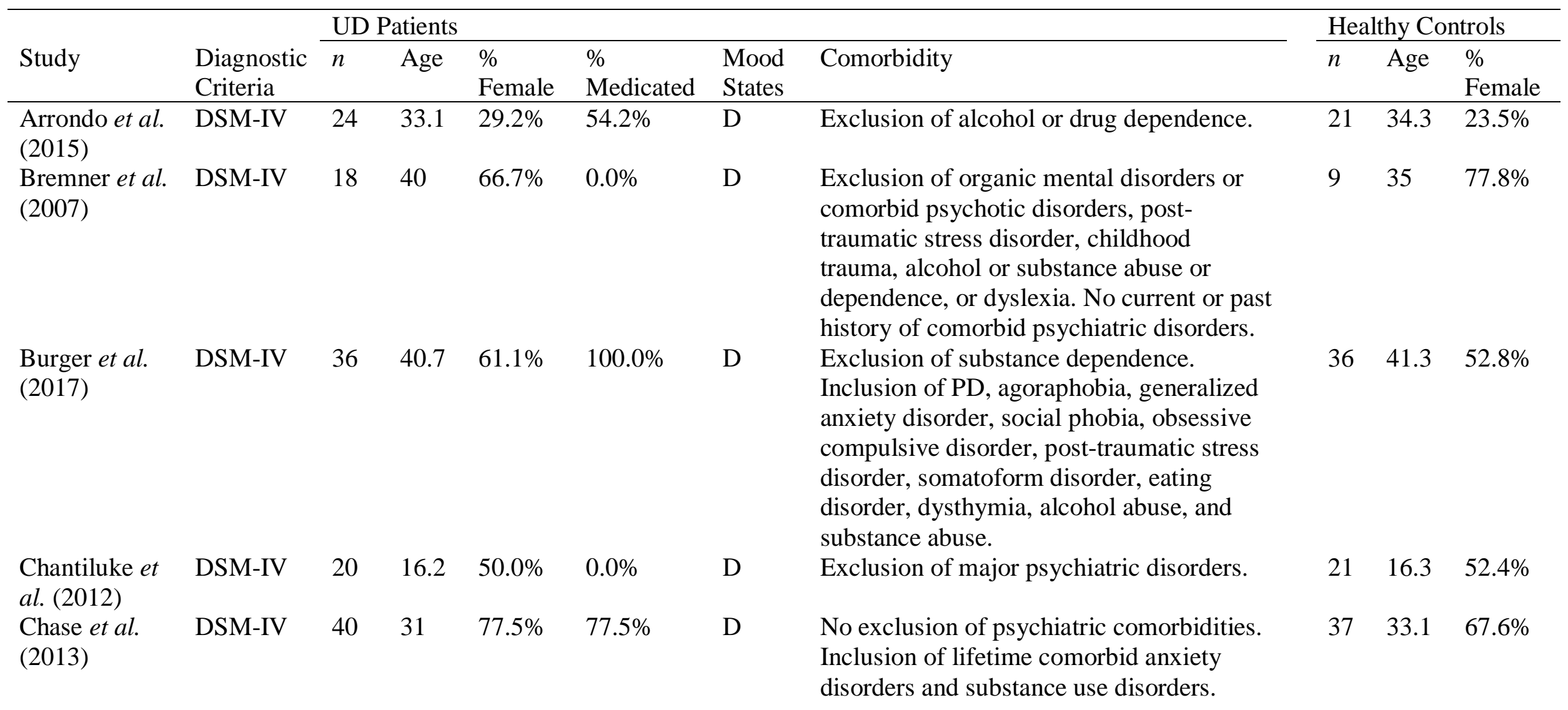


Table 2, continued

\begin{tabular}{|c|c|c|c|c|c|c|c|c|c|c|}
\hline $\begin{array}{l}\text { Demenescu et } \\
\text { al. }(2011)\end{array}$ & DSM-IV & 59 & 36.2 & $66.1 \%$ & $23.7 \%$ & $\mathrm{D}$ & $\begin{array}{l}\text { Exclusion of axis I disorders, such as } \\
\text { psychotic disorder or dementia, current } \\
\text { alcohol or substance abuse. }\end{array}$ & 56 & 39.8 & $60.7 \%$ \\
\hline $\begin{array}{l}\text { Dichter et al. } \\
\text { (2012) }\end{array}$ & DSM-IV & 19 & 23.6 & $78.9 \%$ & $0.0 \%$ & $\mathrm{R}$ & $\begin{array}{l}\text { Exclusion of current axis I } \\
\text { psychopathology. }\end{array}$ & 19 & 27.9 & $63.2 \%$ \\
\hline $\begin{array}{l}\text { Elliott et al. } \\
(2002)\end{array}$ & DSM-IV & 10 & 42.2 & $70.0 \%$ & $100.0 \%$ & $\mathrm{D}$ & $\begin{array}{l}\text { Exclusion of current comorbid anxiety } \\
\text { disorders, substance abuse or dependence, } \\
\text { bipolar disorder, or other psychiatric } \\
\text { diagnoses. Inclusion of past history of PD } \\
\text { and bulimia. }\end{array}$ & 11 & 37.6 & $72.7 \%$ \\
\hline $\begin{array}{l}\text { Engelmann et } \\
\text { al. (2017) }\end{array}$ & DSM-IV & 19 & 37.6 & $52.6 \%$ & $0.0 \%$ & $\mathrm{D}$ & $\begin{array}{l}\text { Exclusion of lifetime bipolar disorder, } \\
\text { psychotic disorder, obsessive-compulsive } \\
\text { disorder, tic disorder, eating disorder, } \\
\text { cognitive disorder, substance abuse or } \\
\text { dependence in the previous } 6 \text { months or } \\
\text { positive urine drug screen, or clinically } \\
\text { significant suicidal ideation. }\end{array}$ & 23 & 33.7 & $60.9 \%$ \\
\hline $\begin{array}{l}\text { Fournier et al. } \\
\text { (2013) }\end{array}$ & DSM-IV & 26 & 30.6 & $69.0 \%$ & $69.2 \%$ & $\mathrm{D}$ & $\begin{array}{l}\text { Exclusion of bipolar disorder, borderline } \\
\text { personality disorder, and alcohol/substance } \\
\text { use disorder within } 2 \text { months before the } \\
\text { scan. Inclusion of history of anxiety } \\
\text { disorder and substance abuse. }\end{array}$ & 28 & 32.6 & $57.0 \%$ \\
\hline $\begin{array}{l}\text { Fu et al. } \\
(2004) \text { and } \\
(2007)\end{array}$ & DSM-IV & 19 & 43.2 & $68.4 \%$ & $100.0 \%$ & $\mathrm{D}$ & $\begin{array}{l}\text { Exclusion of current axis I disorder and } \\
\text { history of substance abuse within } 2 \text { months } \\
\text { of study participation. }\end{array}$ & 19 & 42.8 & $57.9 \%$ \\
\hline
\end{tabular}


Table 2, continued

\begin{tabular}{|c|c|c|c|c|c|c|c|c|c|c|}
\hline $\begin{array}{l}\text { Fu et al. } \\
(2008)\end{array}$ & DSM-IV & 16 & 40 & $81.3 \%$ & $0.0 \%$ & $\mathrm{D}$ & $\begin{array}{l}\text { Exclusion of other axis I disorder, } \\
\text { including anxiety disorder or history of } \\
\text { substance within } 2 \text { months of study } \\
\text { participation. }\end{array}$ & 16 & 39.2 & $81.3 \%$ \\
\hline $\begin{array}{l}\text { Gotlib et al. } \\
\text { (2005) }\end{array}$ & DSM-IV & 18 & 35.2 & $72.2 \%$ & $50.0 \%$ & $\mathrm{D}$ & $\begin{array}{l}\text { Exclusion of psychotic ideation, social } \\
\text { phobia, PD, mania, or substance abuse in } \\
\text { the past } 6 \text { months or behavioral indications } \\
\text { of possible impaired mental status. }\end{array}$ & 18 & 30.8 & $72.2 \%$ \\
\hline $\begin{array}{l}\text { Gradin et al. } \\
\text { (2015) }\end{array}$ & DSM-IV & 25 & 25.5 & $68.0 \%$ & $0.0 \%$ & $\mathrm{D}$ & Unspecified & 25 & 25.4 & $68.0 \%$ \\
\hline $\begin{array}{l}\text { Hall et al. } \\
\text { (2014) }\end{array}$ & DSM-IV & 29 & 37.4 & $55.2 \%$ & $51.7 \%$ & $\mathrm{D}$ & $\begin{array}{l}\text { Exclusion of history of alcohol or } \\
\text { substance abuse. }\end{array}$ & 25 & 37.7 & $55.2 \%$ \\
\hline $\begin{array}{l}\text { Johnston et al. } \\
\text { (2015) }\end{array}$ & $\begin{array}{l}\text { DSM-IV/ } \\
\text { ICD-10 }\end{array}$ & 19 & 50.8 & $78.9 \%$ & $85.0 \%$ & $\mathrm{D}$ & $\begin{array}{l}\text { Exclusion of other primary psychiatric } \\
\text { disorder and substance misuse. }\end{array}$ & 21 & 46.1 & $71.4 \%$ \\
\hline $\begin{array}{l}\text { Keedwell et } \\
\text { al. (2005) }\end{array}$ & ICD-10 & 12 & 43 & $66.7 \%$ & $66.7 \%$ & $\mathrm{D}$ & Exclusion of other axis I disorder. & 12 & 36 & $66.7 \%$ \\
\hline $\begin{array}{l}\text { Knutson et al. } \\
\text { (2008) }\end{array}$ & $\begin{array}{l}\text { DSM-III- } \\
\text { R }\end{array}$ & 14 & 30.7 & $64.3 \%$ & $0.0 \%$ & $\mathrm{D}$ & Exclusion of other current axis I disorder. & 12 & 28.7 & $66.7 \%$ \\
\hline $\begin{array}{l}\text { Kumari et al. } \\
\text { (2003) }\end{array}$ & DSM-IV & 6 & 47 & $100.0 \%$ & $\begin{array}{l}\text { Unspecifie } \\
\mathrm{d}\end{array}$ & $\mathrm{D}$ & Unspecified & 6 & 44 & $100.0 \%$ \\
\hline $\begin{array}{l}\text { Laurent et al. } \\
\text { (2013) }\end{array}$ & DSM-IV & 11 & $\begin{array}{l}24.1 \\
\text { (who } \\
\text { le } \\
\text { sam } \\
\text { ple) }\end{array}$ & $100.0 \%$ & $23.1 \%$ & $\mathrm{D}$ & $\begin{array}{l}\text { No exclusion of psychiatric comorbidities. } \\
\text { Inclusion of past substance } \\
\text { abuse/dependence, anxiety disorders, and } \\
\text { eating disorder. }\end{array}$ & 11 & $\begin{array}{l}24.1 \\
\text { (who } \\
\text { le } \\
\text { sam } \\
\text { ple) }\end{array}$ & $100.0 \%$ \\
\hline Liu et al. & DSM-IV & 21 & 30.7 & $57.1 \%$ & $0.0 \%$ & $\mathrm{D}$ & Exclusion of axis I disorders (other than & 17 & 28.3 & $58.8 \%$ \\
\hline
\end{tabular}

(2017)

anxiety) and psychotic features and

lifetime substance abuse or dependence. 
Table 2, continued

\begin{tabular}{|c|c|c|c|c|c|c|c|c|c|c|}
\hline $\begin{array}{l}\text { Murrough et } \\
\text { al. (2015) }\end{array}$ & DSM-IV & 20 & 38.1 & $44.4 \%$ & $0.0 \%$ & $\mathrm{D}$ & $\begin{array}{l}\text { Exclusion of lifetime history of psychotic } \\
\text { illness or bipolar disorder and current } \\
\text { alcohol or substance abuse. }\end{array}$ & 20 & 35 & $45.0 \%$ \\
\hline $\begin{array}{l}\text { Pizzagalli et } \\
\text { al. (2009) }\end{array}$ & DSM-IV & 30 & 43.2 & $50.0 \%$ & $0.0 \%$ & $\mathrm{D}$ & $\begin{array}{l}\text { Exclusion of other axis I disorder except } \\
\text { for anxiety disorders. }\end{array}$ & 31 & 38.8 & $41.9 \%$ \\
\hline $\begin{array}{l}\text { Remijnse et } \\
\text { al. }(2009)\end{array}$ & DSM-IV & 20 & 35 & $40.0 \%$ & $0.0 \%$ & $\mathrm{D}$ & $\begin{array}{l}\text { Exclusion of current alcohol or substance } \\
\text { abuse at the time of study participation. } \\
\text { Inclusion of social anxiety disorder, } \\
\text { generalized anxiety disorder, PD without } \\
\text { agoraphobia, PD, and cannabis abuse in } \\
\text { early and sustained full remission. }\end{array}$ & 27 & 32 & $70.4 \%$ \\
\hline $\begin{array}{l}\text { Rizvi et al. } \\
\text { (2013) }\end{array}$ & DSM-IV & 21 & 38.9 & $66.7 \%$ & $0.0 \%$ & $\mathrm{D}$ & $\begin{array}{l}\text { Exclusion of other primary axis I disorder, } \\
\text { lifetime history of hypomania/mania, } \\
\text { psychosis, obsessive compulsive disorder, } \\
\text { or eating disorder, and substance abuse or } \\
\text { dependence (except nicotine or caffeine) } \\
\text { within the last } 3 \text { months. }\end{array}$ & 18 & 36.2 & $66.7 \%$ \\
\hline $\begin{array}{l}\text { Schiller et al. } \\
\text { (2013) }\end{array}$ & DSM-IV & 19 & 23.6 & $78.9 \%$ & $0.0 \%$ & $\mathrm{R}$ & $\begin{array}{l}\text { Exclusion of current axis I } \\
\text { psychopathology. }\end{array}$ & 19 & 27.9 & $63.2 \%$ \\
\hline $\begin{array}{l}\text { Segarra et al. } \\
(2016)\end{array}$ & DSM-IV & 24 & 33.1 & $29.2 \%$ & $54.0 \%$ & $\mathrm{D}$ & $\begin{array}{l}\text { Exclusion of dependence on alcohol or } \\
\text { recreational drugs. }\end{array}$ & 21 & 34.3 & $19.0 \%$ \\
\hline
\end{tabular}

(2016) 
Table 2, continued

\begin{tabular}{|c|c|c|c|c|c|}
\hline $\begin{array}{l}\text { Sharp et al. } \\
\text { (2014) }\end{array}$ & DSM-IV & 14 & 13.4 & $100.0 \%$ & $\begin{array}{l}\text { Unspecifie } \\
\text { d }\end{array}$ \\
\hline $\begin{array}{l}\text { Smoski et al. } \\
\text { (2011) }\end{array}$ & DSM-IV & 14 & 34.8 & $50.0 \%$ & $0.0 \%$ \\
\hline $\begin{array}{l}\text { Smoski et al. } \\
\text { (2009) }\end{array}$ & DSM-IV & 9 & 34.4 & $\begin{array}{l}\text { Unspec } \\
\text { ified }\end{array}$ & $44.4 \%$ \\
\hline $\begin{array}{l}\text { Surguladze et } \\
\text { al. (2010) }\end{array}$ & DSM-IV & 16 & 42.3 & $37.5 \%$ & $100.0 \%$ \\
\hline $\begin{array}{l}\text { Surguladze } e t \\
\text { al. (2005) }\end{array}$ & DSM-IV & 9 & 42.8 & $44.4 \%$ & $100.0 \%$ \\
\hline $\begin{array}{l}\text { Townsend et } \\
\text { al. (2010) }\end{array}$ & DSM-IV & 15 & 45.6 & $40.0 \%$ & $0.0 \%$ \\
\hline $\begin{array}{l}\text { Wagner } \text { et al. } \\
\text { (2015) }\end{array}$ & DSM-IV & 19 & 39.9 & $55.0 \%$ & $100.0 \%$ \\
\hline $\begin{array}{l}\text { Wang et al. } \\
\text { (2008) }\end{array}$ & DSM-IV & 12 & 69.1 & $58.3 \%$ & $91.7 \%$ \\
\hline $\begin{array}{l}\text { Young et al. } \\
\text { (2016) }\end{array}$ & $\begin{array}{l}\text { DSM-IV- } \\
\text { TR }\end{array}$ & 16 & 37.1 & $87.5 \%$ & $0.0 \%$ \\
\hline $\begin{array}{l}\text { Zhang et al. } \\
\text { (2017) }\end{array}$ & ICD-10 & 21 & 43.8 & $38.1 \%$ & $100.0 \%$ \\
\hline
\end{tabular}

Exclusion of current use of nicotine, illicit drugs, psychotic disorders, bipolar I

disorder, learning disabilities, and mental retardation.

Exclusion of current mood disorder, anxiety disorder, psychotic disorder,

substance abuse, or active suicidal ideation and history of psychosis or mania.

Inclusion of generalized anxiety disorder and binge eating disorder.

Exclusion of illicit substance abuse.

Exclusion of illicit substance abuse and other axis I disorders.

Exclusion of comorbid axis I disorder.

Exclusion of current comorbid axis I disorder and a history of manic episodes. Exclusion of another major psychiatric disorder and alcohol/drug abuse/dependence. Inclusion of generalized anxiety disorder.

Exclusion of serious suicidal ideation, psychosis, drug/alcohol abuse in the past year and dependence (except for nicotine) in their lifetime.

Exclusion of illicit substance use or substance use disorders.
$19 \quad 13.7 \quad 100.0 \%$

$\begin{array}{lll}15 & 30.8 \quad 60.0 \%\end{array}$

$13 \quad 26.2 \quad$ Unspec ified

$14 \quad 35.1 \quad 42.9 \%$

$9 \quad 39.7 \quad 44.4 \%$

$15 \quad 44.8 \quad 40.0 \%$

$20 \quad 34.1 \quad 60.0 \%$

$20 \quad 73.1 \quad 60.0 \%$

$\begin{array}{lll}16 & 37.8 \quad 87.5 \%\end{array}$

$25 \quad 39.3 \quad 36.0 \%$ 
Table 2, continued

Zhong et al.

DSM-IV

29

$20.5 \quad 55.2 \%$

$0.0 \%$

D

Exclusion of lifetime substance

(2011)

dependence and substance abuse in the last

6 months.

Note. UD, unipolar depression; D, depressed; R, remitted; PD, panic disorder. 
Table 3

Characteristics of the Studies Included in the Meta-analysis

\begin{tabular}{|c|c|c|c|c|c|c|c|}
\hline Study & $\begin{array}{l}\text { fMRI/ } \\
\text { PET }\end{array}$ & Design & Space & Paradigm & Correction & Stimuli & Contrast \\
\hline $\begin{array}{l}\text { Arrondo et al. } \\
\text { (2015) }\end{array}$ & fMRI & $\begin{array}{l}\text { Event- } \\
\text { related }\end{array}$ & MNI & $\begin{array}{l}\text { Modified monetary } \\
\text { incentive delay task }\end{array}$ & Uncorrected & Money & $\begin{array}{l}\text { HC > UD, Anticipation: } \\
\text { Reward > Non-Reward }\end{array}$ \\
\hline $\begin{array}{l}\text { Bremner et al. } \\
(2007)\end{array}$ & PET & Block & MNI & $\begin{array}{l}\text { Verbal declarative } \\
\text { memory tasks with } \\
\text { neutral paragraph } \\
\text { encoding compared to } \\
\text { a control condition and } \\
\text { sad word pair retrieval } \\
\text { compared to a control } \\
\text { condition. }\end{array}$ & $\begin{array}{l}\text { Uncorrected at } \mathrm{p} \\
<.005\end{array}$ & $\begin{array}{l}\text { Words and } \\
\text { paragraphs }\end{array}$ & $\begin{array}{l}\text { UD > HC, Outcome: Negative } \\
>\text { Neutral } \\
\text { HC > UD, Outcome: Negative } \\
>\text { Neutral }\end{array}$ \\
\hline $\begin{array}{l}\text { Burger et al. } \\
\text { (2017) }\end{array}$ & fMRI & $\begin{array}{l}\text { Event- } \\
\text { related }\end{array}$ & MNI & $\begin{array}{l}\text { Face matching } \\
\text { paradigm }\end{array}$ & $\begin{array}{l}\text { Corrected at } \mathrm{p}<.05 \\
\text { (TFCE) }\end{array}$ & Faces & $\begin{array}{l}\text { HC > UD, Outcome: Negative } \\
>\text { Neutral } \\
\text { HC > UD, Outcome: Positive } \\
>\text { Neutral }\end{array}$ \\
\hline $\begin{array}{l}\text { Chantiluke } \text { et al. } \\
\text { (2012) }\end{array}$ & fMRI & $\begin{array}{l}\text { Event- } \\
\text { related }\end{array}$ & TAL & $\begin{array}{l}\text { Reward continuous } \\
\text { performance task }\end{array}$ & $\begin{array}{l}\text { Uncorrected at } p \\
<.005\end{array}$ & Money & $\begin{array}{l}\text { UD > HC, Outcome: Reward > } \\
\text { Non-Reward } \\
\text { HC > UD, Outcome: Reward > } \\
\text { Non-Reward }\end{array}$ \\
\hline
\end{tabular}


Table 3, continued

Chase et al.

(2013)

fMRI

Event-
related
MNI Card guessing paradigm

Voxel-wise corrected at $\mathrm{p}<.05$ and cluster-wise corrected at $\mathrm{p}<.01$

Demenescu et al. fMRI (2011)

Eventrelated

MNI Viewing faces with angry, fearful, sad, happy, and neutral expressions and scrambled faces; rating gender or pressing buttons in conformity with the instruction presented on the

\section{screen}

Dichter et al.

fMRI

(2012)
Eventrelated

\section{MNI Modified monetary} incentive delay task
Cluster-wise corrected at $\mathrm{p}<.05$

Uncorrected at $\mathrm{p}$ $<.005, \mathrm{k} \geq 10$
Money

UD > HC, Anticipation: Reward > Non-Reward HC > UD, Anticipation: Reward > Non-Reward UD > HC, Anticipation: Reward Expectancy HC > UD, Anticipation:

Reward Expectancy

UD > HC, Outcome:

Prediction Error

UD > HC, Outcome: Positive

$>$ Scrambled Face

UD > HC, Anticipation:

Reward > Non-Reward

UD > HC, Outcome: Reward > Non-Reward

HC > UD, Outcome: Reward > Non-Reward 
Table 3, continued

Elliott et al.

(2002)

Engelmann et al. (2017)

Fournier et al.

(2013)

Fu et al. (2004) and (2007)

Fu et al. (2008)
fMRI

Eventrelated

fMRI Block

fMRI

fMRI

Event-

related

Block

Eventrelated
MNI Affective go/no go task

Uncorrected at $\mathrm{p}$

$<.001$

MNI Economic decision-

making task

MNI Labeling a color flash

superimposed upon

neutral faces that

gradually morphed

into angry, fearful, sad,

or happy faces

TAL Indicating the sex of

faces morphed to

represent low,

medium, and high

intensities of sadness

TAL Indicating the sex of

faces morphed to

represent low,

medium, and high

intensities of sadness
Cluster-wise Money

corrected at $\mathrm{p}<.05$

Uncorrected at $\mathrm{p} \quad$ Faces

$<.001, \mathrm{k}>20$

\section{Cluster-wise}

corrected at $\mathrm{p}<.005$

Faces

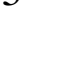

Unspecified Faces

Faces

UD > HC, Outcome: Negative $>$ Positive

HC > UD, Outcome: Positive

$>$ Negative

UD > HC, Outcome: Negative

$>$ Positive

UD > HC, Outcome: Negative

$>$ Neutral UD > HC, Outcome:

Positive $>$ Neutral

UD > HC, Outcome: Negative (low, medium, and high

intensity)

HC > UD, Outcome: Positive

(low, medium, and high

intensity)

UD > HC, Outcome: Negative

(low, medium, and high

intensity)

HC > UD, Outcome: Negative

(low, medium, and high intensity) 
Table 3, continued

Gotlib et al.

(2005)

fMRI Block

MN

Indicating the sex of
faces that were fearfu

Uncorrected at $\mathrm{p}$ angry, sad, happy,

neutral, or scrambled

$<.001, \mathrm{k}>5$

Faces
Gradin et al.
(2015)

fMRI

Event-
related

MNI Ultimatum game

Hall et al. (2014)

fMRI
TAL Contingency reversal
reward paradigm related

\section{Cluster-wise \\ corrected at $\mathrm{p}<.05$}

Voxel-wise
corrected at $\mathrm{p}<.05$
UD > HC, Outcome: Negative

$>$ Neutral

HC > UD, Outcome: Negative

$>$ Neutral

UD > HC, Outcome: Positive

$>$ Neutral

HC > UD, Outcome: Positive

$>$ Neutral

HC > UD, Outcome:

Increasing fairness (decreasing inequality)

UD > HC, Outcome:

Increasing inequality

(decreasing fairness)

HC > UD, Outcome:

Magnitude of Loss: Large Loss

$>$ Small Loss

HC > UD, Outcome:

Magnitude of Reward: Large

Reward > Small Reward

UD > HC, Outcome: Reward

Acquisition > Punishment

Reversal

HC > UD, Outcome: Reward

Acquisition $>$ Punishment

Reversal 
Table 3, continued Johnston et al. (2015)

fMRI

Eventrelated
MNI Modified Pessiglione
task

Keedwell et al.

fMRI

(2005)

Knutson et al.

(2008)
fMRI Event-

related
TAL Being exposed to happy, sad, or neutral autobiographical facial expressions

TAL Monetary incentive delay task memory prompts and
Cluster-wise

corrected at $\mathrm{p}<.01$

Cluster-wise

Voucher

UD > HC, Outcome: Loss >

Non-Loss

HC > UD, Outcome: Loss >

Non-Loss

UD > HC, Outcome: Reward >

Non-Reward

HC > UD, Outcome: Reward >

Non-Reward

corrected at $\mathrm{p}<.01$

Autobiogra UD > HC, Outcome: Negative

phical > Neutral

memory $\quad H C>$ UD, Outcome: Negative

and faces $>$ Neutral

UD > HC, Outcome: Positive

$>$ Neutral

HC > UD, Outcome: Positive

$>$ Neutral

UD > HC, Anticipation:

Uncorrected at $\mathrm{p} \quad$ Money

$<.05$

Reward > Non-Reward

HC > UD, Anticipation:

Reward $>$ Non-Reward

HC > UD, Outcome: Non-Loss

$>$ Loss

HC > UD, Outcome: Reward >

Non-Reward 
Table 3, continued

Kumari et al. (2003)

fMRI Block
TAL Viewing positive or negative pictures with a caption
Cluster-wise corrected at $\mathrm{p}<.005$

\section{Laurent et al. (2013)}

Liu et al. (2017)

fMRI

Eventrelated

MNI Seeing own infant vs. other infant distress faces

fMRI

Eventrelated

Murrough et al. (2015)

fMRI Event-
related

\section{$\mathrm{MN}$}

Instrumental probabilistic rewardand punishment-based associative learning task

\section{MNI Rating emotional} valence of happy, sad, or neutral faces
Cluster-wise

Pictures $\quad$ HC $>$ UD, Outcome: Negative and captions UD > HC, Outcome: Negative $>$ Neutral

HC > UD, Outcome: Positive

$>$ Neutral

UD > HC, Outcome: Positive

$>$ Neutral

HC > UD, Outcome: Positive

$>$ Negative

UD > HC, Outcome: Positive

$>$ Negative

HC > UD, Outcome: Very

negative $>$ Negative

\section{Cluster-wise \\ Money}

corrected at $\mathrm{p}<.05$

UD > HC, Outcome: Negative $>$ Neutral

UD > HC, Outcome:

Punishment Prediction Errors

\section{Cluster-wise \\ Faces}

HC > UD, Outcome: $100 \%$

Positive > Neutral 
Table 3, continued Pizzagalli et al. (2009)

fMRI

Eventrelated

MNI Monetary incentive delay task

Uncorrected at $\mathrm{p}$ $<.005$

Remijnse et al. (2009)

fMRI Eventrelated

MNI Reversal learning task

Uncorrected $\mathrm{p}$

$<.001$

Rizvi et al.

(2013)
fMR
Blocked MNI

\section{Cluster-wise}

$\begin{array}{ll}\text { Viewing IAPS pictures } & \text { Cluster-wise } \\ \text { that elicit positive, } & \text { corrected at } p<.05 \\ \text { negative or neutral } & \\ \text { affective states } & \end{array}$

Money

UD > HC, Anticipation: Loss

$>$ Non-Loss

HC > UD, Anticipation: Loss

$>$ Non-Loss

UD > HC, Anticipation:

Reward > Non-Reward

HC > UD, Anticipation:

Reward $>$ Non-Reward

UD > HC, Outcome: Loss >

Non-Loss

HC > UD, Outcome: Loss >

Non-Loss

UD > HC, Outcome: Reward > Non-Reward

HC > UD, Outcome: Reward >

Non-Reward

UD > HC, Outcome: Loss >

Baseline

HC > UD, Outcome: Loss >

Baseline

UD > HC, Outcome: Reward >

Baseline

Pictures UD > HC, Outcome: Positive

$>$ Neutral

UD > HC, Outcome: Negative

$>$ Neutral 
Table 3, continued Rosenblau et al. (2012)

fMRI

Event-
related

Scheuerecker $e t$ al. (2010)

Schiller et al. (2013)

Segarra et al. (2016)

Sharp et al. (2014)

fMRI Event-
MNI that elicit positive, negative or neutral and without cues indicating their emotional valence

\section{MNI Face matching} paradigm related

MNI Monetary incentive

delay task

Uncorrected at $\mathrm{p}$ $<.001$

Cluster-wise

corrected at $\mathrm{p}<.05$

Money

\section{Cluster-wise}

related machine game

\section{TAL Card guessing} paradigm

related corrected at $\mathrm{p}<.05$
Uncorrected at $\mathrm{p}$

$<.005$
UD > HC, Anticipation:

Negative $>$ Neutral

UD > HC, Outcome: Negative

$>$ Neutral

UD > HC, Outcome: Negative $>$ Neutral

HC > UD, Anticipation: Loss

$>$ Non-Loss

HC > UD, Outcome: Loss >

Non-Loss

$\mathrm{HC}>\mathrm{UD}$, Outcome:

Unexpected Reward > Full

Miss
HC > UD, Outcome: Reward > Non-Reward 
Table 3, continued

Smoski et al.

(2011)

fMRI

related

MNI

Modified monetary

incentive delay task

Cluster-wise

corrected

Money

Uncorrected at $\mathrm{p}$

$<.005, \mathrm{k} \geq 10$

Money

(2009)

Surguladze et al. fMRI Event(2010) related

MNI Wheel of fortune task related

\section{TA}
TAL Indicating the sex of Cluster-wise neutral faces and faces corrected at $\mathrm{p}<.001$ morphed to represent mild and high intensities of fear and disgust

UD > HC, Anticipation: Money $>$ Control

HC > UD, Anticipation:

Money $>$ Control

UD > HC, Outcome: Non-Win

$>$ Control

HC > UD, Outcome: Non-Win

$>$ Control

UD > HC, Outcome: Winning

$>$ Control

HC > UD, Outcome: Winning

$>$ Control

UD > HC, Selection: Money >

Control

HC > UD, Selection: Money >

Control

$\mathrm{HC}>\mathrm{UD}$, Anticipation:

Reward > Non-Reward

HC > UD, Outcome: Reward >

Non-Reward

$\mathrm{HC}>\mathrm{UD}$, Outcome:

Increasing intensities of happy

faces

UD > HC, Outcome:

Increasing intensities of sad faces 
Table 3, continued Surguladze et al. (2005)

fMRI Eventrelated

Townsend et al.

(2010)

Wagner et al.

(2015)

Wang et al. (2008)

Young et al.

(2016)

fMRI

fMRI

Event-

related

Event-

related

Zhang et al.

(2017)

fMRI Event-

\section{TAL Indicating the sex of}

\section{MNI Face matching}

\section{MNI Self-referential}

MNI Emotional oddball task

\section{TAL Autobiographical} neutral faces and faces morphed to represent mild and high intensities of sadness and happiness paradigm processing task

\section{Cluster-wise \\ corrected at $\mathrm{p}<.05$ \\ Cluster-wise}

corrected at $\mathrm{p}<.05$ memory task

\section{Cluster-wise \\ Faces \\ corrected at $p<.001$}

Uncorrected at $\mathrm{p}$

$<.001, \mathrm{k}=5$

Cluster-wise

corrected at $\mathrm{p}<.05$, $\mathrm{k}>30$

\section{Cluster-wise} corrected at $\mathrm{p}<.05$, positive, neutral, and negative pictures with $\mathrm{k}>157$
UD > HC Outcome:

Differential response to $100 \%$ disgust

HC > UD, Outcome:

Differential response to $50 \%$ fear

Faces $\quad$ HC $>$ UD, Outcome: Negative $>$ Neutral

Statements UD > HC, Outcome: Neutral > Negative

UD > HC, Outcome: Neutral > Positive

Pictures UD > HC, Outcome: Negative $>$ Neutral

Words and HC $>$ UD, Outcome: Very

autobiogra Positive $>$ Positive

phical HC > UD, Outcome: Very

memories Negative $>$ Negative

UD > HC, Outcome: Very

Negative $>$ Negative

Pictures $\quad$ UD $>$ HC, Outcome: Reward > Non-Reward 
Table 3, continued

Zhong et al.

(2011)

fMRI Block MNI $\begin{aligned} & \text { Face matching } \\ & \text { paradigm }\end{aligned} \quad \begin{aligned} & \text { Uncorrected at } \mathrm{p} \\ & <.005, \mathrm{k}=8\end{aligned} \quad$ Faces

UD > HC, Outcome: Negative $>$ Neutral

HC > UD, Outcome: Negative $>$ Neutral

Note. fMRI, functional magnetic resonance imaging; PET, positron emission tomography; MNI, Montreal Neurological Institute space; SVC, small volume correction; UD, unipolar depression; HC, healthy controls; TFCE, threshold-free cluster enhancement; TAL, Talairach space; VS, ventral striatum; dACC, dorsal anterior cingulate cortex; rACC, rostral anterior cingulate cortex; ACC, anterior cingulate cortex; mPFC, medial prefrontal cortex; mOFC, medial orbitofrontal cortex; IAPS, International Affective Picture System. 
In the present meta-analytic dataset, for the UD group, the mean number of participants was 19.9 , the mean age was 36.4 , the mean percentage of females was $60.9 \%$, and the mean percentage of medication usage was $36.6 \%$. For the HC group, the mean number of participants was 20.1 , the mean age was 34.9 , and the mean percentage of females was $60.3 \%$. Types of reward or punishment used by the included studies encompass money, points, or voucher $(41.5 \%$; 17/41); faces $(34.1 \%$; $14 / 41)$; pictures $(12.2 \% ; 5 / 41)$; words, statements, captions, or paragraphs $(12.2 \%$; 5/41); and autobiographical memory $(4.9 \% ; 2 / 41)$. Both reward and punishment contrasts were reported in $41.5 \%(17 / 41)$ of studies; $29.3 \%(12 / 41)$ of studies reported punishment contrasts only; and 26.8\% (11/41) of studies reported reward contrasts only.

I first synthesized results of 22 studies reporting less activity in response to reward in people with UD than $\mathrm{HC}$ (i.e. HC > UD for reward > punishment/neutral stimuli and/or neutral stimuli > punishment). As expected, results indicated that these studies reliably reported less activation in a single cluster extending bilaterally across the VS and including part of the subcallosal cortex in UD (Table 4; Figure 3A).

In addition to examining which regions consistently showed hypo-responses to reward, I also examined which, if any, brain regions showed consistent hyperresponses to rewarding stimuli. I aggregated results of 18 studies reporting greater activity in response to reward in people with UD than $\mathrm{HC}$ (i.e. UD > HC for reward > punishment/neutral stimuli and/or neutral stimuli > punishment). Importantly, results indicated that these studies reliably reported greater activation in the right OFC (in a region located in between the medial OFC and the lateral OFC) in UD (Table 4; Figure 3B). 
Table 4

Peak Coordinates of Group Differences in Neural Responses to Reward

\begin{tabular}{lclccc}
\hline Contrast & $\begin{array}{c}\text { Cluster Size } \\
\left(\mathrm{mm}^{3}\right)\end{array}$ & $\begin{array}{l}\text { Probabilistic Anatomical } \\
\text { Label }\end{array}$ & $\mathrm{x}$ & $\mathrm{y}$ & $\mathrm{z}$ \\
\hline $\mathrm{UD}>\mathrm{HC}$ & 912 & Frontal Orbital Cortex & 20 & 32 & -12 \\
& & $(26 \%)$, Frontal Pole (13\%) & & & \\
$\mathrm{HC}>\mathrm{UD}$ & \multirow{2}{*}{1768} & Subcallosal Cortex (14\%) & -2 & 8 & -4 \\
& & Caudate (32.1\%), & 8 & -2 \\
& & Accumbens (11.1\%) & & \\
\hline
\end{tabular}

Note. Coordinates are $\mathrm{x}, \mathrm{y}, \mathrm{z}$ values of the locations of the maximum activation

likelihood estimation (ALE) values in MNI space. Probabilistic labels reflect the probability that a coordinate belongs to a given region derived from the Harvard-

Oxford probabilistic atlas. For clarity, I only report labels whose likelihood exceeds 5\%. UD, unipolar depression; HC, healthy controls. 
A Hypo Response to Reward in the VS

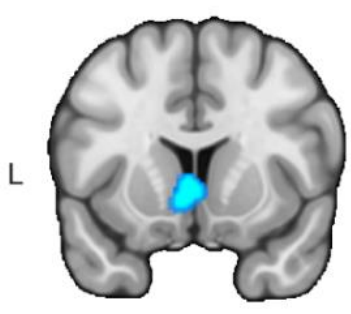

$Y=12$

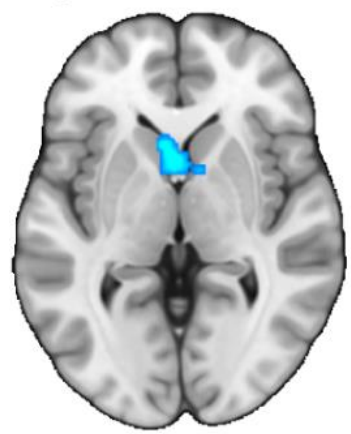

$Z=1$

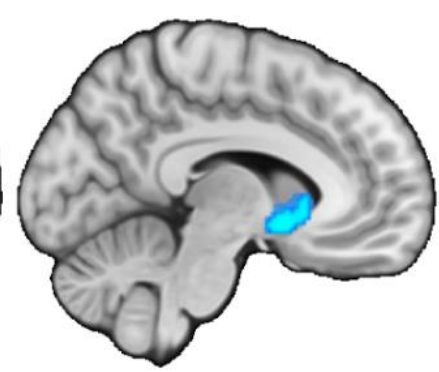

$X=-8$

B

Hyper Response to Reward in the OFC

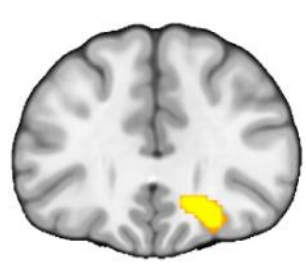

$Y=32$

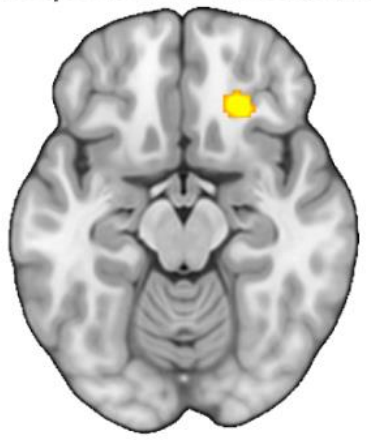

$Z=-15$

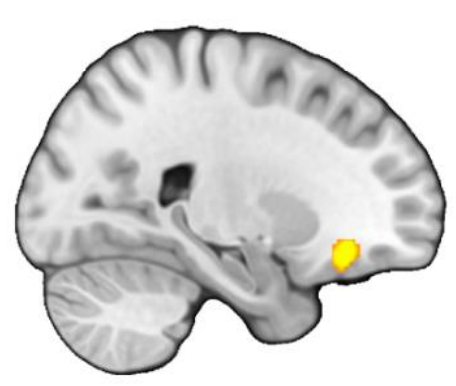

$x=23$

Figure 3. Opposing abnormalities in the reward circuit in response to reward in unipolar depression. (A) To examine regions that consistently showed blunted response to reward, 22 studies reporting less activity in response to reward in people with unipolar depression (UD) than healthy controls (HC) were synthesized. Results indicated that these studies reliably report less activation in the ventral striatum (VS) in UD. (B) To identify regions that consistently showed hyper-responses to reward, 18 studies reporting greater activity in response to reward in people with UD than HC were meta-analyzed. Results indicated that these studies reliably report greater activation in the right orbitofrontal cortex (OFC) in UD. 
I conducted sensitivity analyses to examine whether excluding studies that used neutral stimuli > punishment as a reward contrast would affect the main results related to reward responses in UD. After excluding the two experiments of neutral stimuli > punishment (Demenescu et al., 2011; Wagner et al., 2015), the results remained the same: I found significant convergence among experiments reporting blunted responses for reward in UD relative to $\mathrm{HC}$ in the VS, as well as significant convergence among experiments reporting elevated responses for reward in UD relative to $\mathrm{HC}$ in the $\mathrm{OFC}$ (Table 5).

Table 5

Peak Coordinates of Group Differences in Neural Responses to Reward (Excluding Neutral Stimuli > Punishment)

\begin{tabular}{lclccc}
\hline Contrast & $\begin{array}{c}\text { Cluster Size } \\
\left(\mathrm{mm}^{3}\right)\end{array}$ & $\begin{array}{l}\text { Probabilistic Anatomical } \\
\text { Label }\end{array}$ & $\mathrm{x}$ & $\mathrm{y}$ & $\mathrm{z}$ \\
\hline $\mathrm{UD}>\mathrm{HC}$ & 968 & Frontal Orbital Cortex & 20 & 32 & -12 \\
& & $(26 \%)$, Frontal Pole $(13 \%)$ & & & \\
$\mathrm{HC}>\mathrm{UD}$ & 1784 & Subcallosal Cortex (14\%) & -2 & 8 & -4 \\
& & Caudate (32.1\%), & 6 & -2 \\
& & Accumbens (11.1\%) & & \\
\hline
\end{tabular}

Note. Coordinates are $\mathrm{x}, \mathrm{y}, \mathrm{z}$ values of the locations of the maximum activation likelihood estimation (ALE) values in MNI space. Probabilistic labels reflect the probability that a coordinate belongs to a given region derived from the HarvardOxford probabilistic atlas. For clarity, I only report labels whose likelihood exceeds 5\%. UD, unipolar depression; HC, healthy controls.

I also conducted exploratory analyses to examine which brain regions consistently show aberrant responses to punishment in UD relative to HC. First, I meta-analyzed 24 studies reporting greater activity in response to punishment in people with UD than HC (i.e. UD > HC for punishment > reward/neutral stimuli 
and/or neutral stimuli > reward). The results indicated that these studies reliably reported greater activation in the left sublenticular extended amygdala in UD (Table 6; Figure 4). Second, I synthesized 17 studies reporting less activity in response to punishment in people with UD than HC (i.e. HC > UD for punishment > reward/neutral stimuli and/or neutral stimuli > reward). The results indicated that these studies did not report consistent activation patterns. Together, these results suggest that relative to HC, people with UD exhibited hyper-responses in the left sublenticular extended amygdala during processing of punishment-relevant stimuli.

Table 6

Peak Coordinates of Group Differences in Neural Responses to Punishment

\begin{tabular}{lclccc}
\hline Contrast & $\begin{array}{c}\text { Cluster Size } \\
\left(\mathrm{mm}^{3}\right)\end{array}$ & $\begin{array}{l}\text { Probabilistic Anatomical } \\
\text { Label }\end{array}$ & $\mathrm{x}$ & $\mathrm{y}$ & $\mathrm{z}$ \\
\hline UD $>$ HC & 1104 & Amygdala (85.4\%) & -26 & -8 & -14 \\
& & Amygdala (61.4\%) & -16 & -2 & -18 \\
\hline
\end{tabular}

Note. Coordinates are $\mathrm{x}, \mathrm{y}, \mathrm{z}$ values of the locations of the maximum activation

likelihood estimation (ALE) values in MNI space. Probabilistic labels reflect the probability that a coordinate belongs to a given region derived from the HarvardOxford probabilistic atlas. For clarity, I only report labels whose likelihood exceeds 5\%. UD, unipolar depression; HC, healthy controls. 


\section{Hyper Response to Punishment in the SLEA}

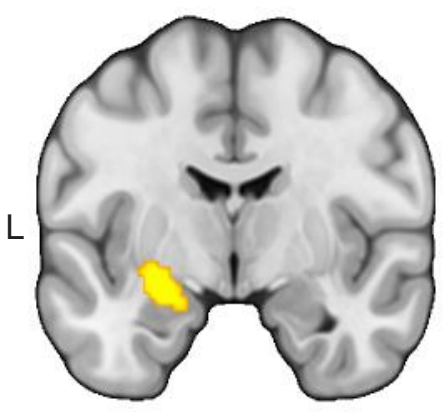

$Y=-6$

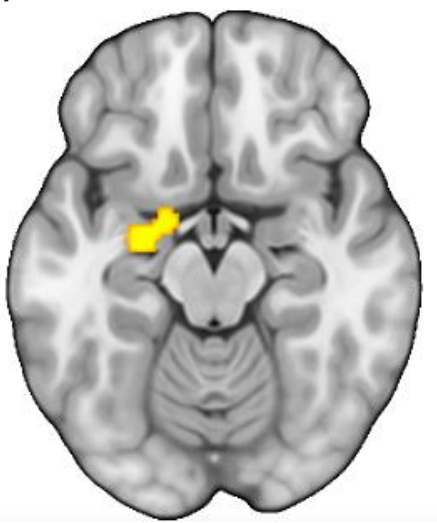

$Z=-16$

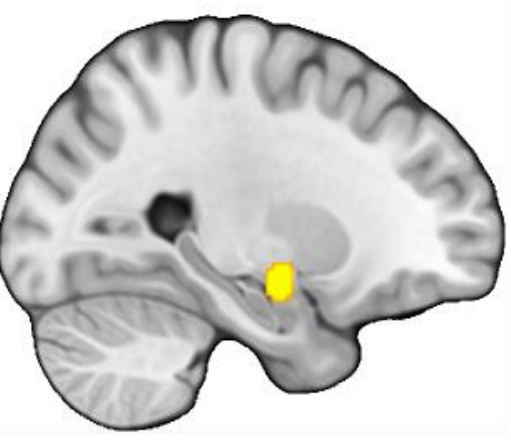

$X=-26$

Figure 4. Hyper-responses to punishment in the sublenticular extended amygdala in unipolar depression. To conduct exploratory analyses to examine which brain regions consistently show elevated response to punishment in unipolar depression (UD) relative to healthy controls (HC), I meta-analyzed 24 studies reporting greater activity in response to punishment in people with UD than HC. The results indicated that these studies reliably report greater activation in the left sublenticular extended amygdala (SLEA) in UD.

\section{Discussion}

A growing number of researchers have used neuroimaging methods to enhance our understanding of the underlying pathophysiology of mood disorders (Hamilton et al., 2012; C. H. Miller, Hamilton, Sacchet, \& Gotlib, 2015; Palmer, Crewther, Carey, \& START Project Team, 2014). Many of these studies have shown that patients with mood disorders exhibit abnormal responses in the VS, but more disparate patterns of responses in other brain areas. Therefore, it remains unclear what brain regions, other than the VS, are most consistently implicated in people with mood disorders, particularly during reward processing (see Table 1 and Figure 1). To address this issue, I aimed to perform a coordinate-based meta-analysis that synthesized studies on reward processing dysfunction in mood disorders. 
Nevertheless, as there was an insufficient number of studies focusing on BD, the current meta-analysis focused on 41 neuroimaging studies containing reward-related responses from a total of 794 patients with UD and $803 \mathrm{HC}$. The meta-analytic findings suggested that reward responses within the VS are consistently blunted in UD relative to $\mathrm{HC}$ across studies. In contrast, I found that reward responses within the OFC are consistently elevated in UD. Contrary to the common notion that UD is characterized by blunted responses to reward, these findings suggest that UD may be characterized by both hypo- and hyper-responses to reward at the neural level and highlight the need for a more fine-tuned understanding of the various components of reward processing in UD.

Although the current study's blunted striatal findings are consistent with previous meta-analytic work documenting reward processing abnormalities in UD (Groenewold et al., 2013; Keren et al., 2018; W. N. Zhang et al., 2013), I emphasize that the current work differs in two key ways. First, the present results implicate highly specific — yet distinct—abnormalities in the reward circuit, with hyporesponses to reward in the VS and hyper-responses to reward in the OFC. In sharp contrast, previous meta-analyses have generally reported distributed patterns of abnormalities, with little anatomical agreement across studies (see Table 1 and Figure 1). Second, to minimize bias, the current study employed more stringent analysis methods than prior studies in this area, following recommendations from new guidelines (Müller, Cieslik, Laird, et al., 2017; Müller, Cieslik, Serbanescu, et al., 2017). For example, instead of using the FDR approach, which has been shown to be inadequate in controlling the false positives among clusters in neuroimaging metaanalyses (Chumbley \& Friston, 2009; Eickhoff et al., 2012), I corrected for multiple comparisons using the permutation-based approach. I also excluded ROI- or SVC- 
based studies and only included whole-brain studies that used an active control condition and independent samples. In addition, I only conducted a meta-analysis when there were at least 17 eligible experiments to restrict excessive contribution of any particular studies to cluster-level thresholding (Eickhoff et al., 2016). I speculate that the enhanced rigor and methods of the current study contributed to its ability to identify highly circumscribed and distinct abnormalities in the reward circuit.

A prior meta-analysis using similarly rigorous methods revealed no significant convergence of findings among neuroimaging studies comparing UD and HC (Müller, Cieslik, Serbanescu, et al., 2017). Nevertheless, I note that the previous meta-analysis differed from the current meta-analysis in at least four key ways. First, whereas the previous meta-analysis focused on emotional or cognitive processing, the current meta-analysis focused solely on reward processing. Second, the previous metaanalysis excluded participants younger than 18 years old; in contrast, the current meta-analysis included participants of all ages, boosting its power and ability to generalize its findings to UD across ages. Third, the previous meta-analysis included studies up until October 2015, whereas the current meta-analysis included studies until August 2018. Finally, the previous meta-analysis excluded UD participants in remission, whereas the current meta-analysis included them, leading to the question of whether reward processing dysfunction is not simply a state, but a trait of UD. This issue needs to be addressed explicitly when there are enough studies available to compare current to remitted depression. Our ability to identify significant convergence highlights the significance of reward processing dysfunction in UD and might indicate the literature on reward processing in UD is more homogeneous than that on emotional or cognitive processing in UD. 
In my view, the most important current finding is that studies consistently report that people with UD exhibit hyper-responses to reward in an OFC region located between the medial OFC and the lateral OFC (Hare, O'Doherty, Camerer, Schultz, \& Rangel, 2008). Exposure to rewards (e.g., money and pleasant sights) evokes activity in the OFC, which has been associated with the computation of reward value (Berridge \& Kringelbach, 2015; Der-Avakian \& Markou, 2012; Kringelbach, 2005; Levy \& Glimcher, 2012; Padoa-Schioppa, 2011; Padoa-Schioppa \& Conen, 2017; Rangel et al., 2008; Saez et al., 2017; Stalnaker et al., 2015; K. S. Wang et al., 2016) as well as the representation of current stimulus-outcome associations (Pickens et al., 2003; Sharpe et al., 2019). For example, whereas the lateral OFC is responsive to stimuli of both positive and negative valence, the medial OFC is particularly responsive to cues of positive valence (Kringelbach \& Rolls, 2004; Lewis, Critchley, Rotshtein, \& Dolan, 2007; Nusslock \& Alloy, 2017; Schmidt et al., 2009). Therefore, given that UD is traditionally linked to blunted response to reward or reduced capacity to experience pleasure (Whitton et al., 2015), the finding of hyperactivity of the OFC in response to reward in UD may seem paradoxical. One interpretation would be that UD is at least partly characterized by hyper-responses to reward, which fits with a set of experimental studies reporting that individuals with severe UD found dextroamphetamine to be more rewarding than did controls (Naranjo, Tremblay, \& Busto, 2001; Tremblay, Naranjo, Cardenas, Herrmann, \& Busto, 2002; Tremblay et al., 2005).

Alternatively, OFC hyperactivity may reflect enhanced inhibitory control over subcortical regions underlying reward-related behavior, leading to anhedonia. Optogenetic and neuroimaging studies have revealed that hyperactivity in prefrontal regions (e.g., medial PFC, vmPFC) innervated by glutamatergic neurons may causally 
inhibit reward-related behavior via suppressing striatal responses to dopamine neurons in the midbrain (Ferenczi et al., 2016; Robbins, 2016) and increasing connectivity between the medial PFC, lateral OFC, and VS (Ferenczi et al., 2016; Robbins, 2016). These findings from animal studies suggest that prefrontal regions (e.g., medial PFC, vmPFC, and lateral OFC) might exert inhibitory control over subcortical regions underlying reward-relevant behavior, potentially explaining the central role of anhedonia in UD. Nevertheless, it is important to note that given the complexity of the OFC and the inability of the meta-analysis to unravel its varied functions, these interpretations remain speculative.

The extent to which corticostriatal connectivity during reward processing is disrupted in UD remains an open and important question (Admon \& Pizzagalli, 2015b; Drysdale et al., 2017; Kaiser, Andrews-Hanna, Wager, \& Pizzagalli, 2015). Previous meta-analyses indicate that at least some people with UD exhibit dysfunction in resting-state corticostriatal connectivity (Drysdale et al., 2017; Kaiser et al., 2015). The current meta-analytic results will provide a springboard for future studies that seek to develop a full picture of the pathophysiology of UD and understand the role of dysregulated corticostriatal connectivity in UD, particularly in the context of reward processing. These endeavors may require empirical assessments of connectivity within the reward circuit using psychophysiological interaction analysis (PPI; Friston et al., 1997; McLaren, Ries, Xu, \& Johnson, 2012; D. V. Smith, Gseir, Speer, \& Delgado, 2016) and dynamic causal modeling (Friston, Harrison, \& Penny, 2003). Such approaches have shown promise for revealing specific patterns of task-dependent corticostriatal interactions in samples containing healthy individuals (Chatham, Frank, \& Badre, 2014; D. V. Smith, Rigney, \& Delgado, 2016; Wimmer, Daw, \& Shohamy, 2012; Wimmer \& Shohamy, 2012), clinical populations (Admon 
\& Pizzagalli, 2015b, 2015a; K. D. Young et al., 2016), or a mix of both (Hanson, Knodt, Brigidi, \& Hariri, 2017). Nevertheless, a caveat of such approaches is that dysregulated corticostriatal connectivity may involve modulatory regions, such as the midbrain (Murty et al., 2014).

In addition to distinct abnormalities within the reward circuit, the current study also found that UD is associated with hyper-responses in the left sublenticular extended amygdala in response to punishment. The current finding fits with others in suggesting that amygdala hyperactivation is linked to the processing of affectively salient, especially punishing, stimuli in UD, and may underlie negativity bias in depression (Eshel \& Roiser, 2010; Roiser, Elliott, \& Sahakian, 2012). It is also in agreement with a meta-analysis indicating increased activation in the amygdala in response to negative stimuli in UD relative to HC (Hamilton et al., 2012) and a series of studies indicating that the amygdala may be a key brain region implicated in the pathophysiology of depression (Phillips, Drevets, Rauch, \& Lane, 2003; Price \& Drevets, 2010; Rive et al., 2013). Interestingly, longitudinal studies have reported that amygdala reactivity, potentially in combination with life stress, is prospectively associated with internalizing (e.g., depressive and anxiety) symptoms (Mattson, Hyde, Shaw, Forbes, \& Monk, 2016; Swartz, Knodt, Radtke, \& Hariri, 2015), highlighting the importance of amygdala reactivity in the course of depression.

Even though the current meta-analysis reveals circumscribed patterns of abnormal responses to reward and punishment, I note that the findings should be interpreted in the context of their limitations. First, heterogeneity across studies may have added noise to the current analyses and restricted its capacity for detecting true effects. Specifically, due to the limited number of studies, the current analyses collapsed across different reward processes (e.g., anticipation and outcome), reward 
modalities (e.g., monetary and social), and specific contrasts that would help isolate and differentiate neural responses to salience and valence (Cooper \& Knutson, 2008). The current analyses also collapsed across different mood states, psychotropic medication usage, ages, and comorbidities. In doing so, important differences in brain activation may be obscured and more specific questions related to brain activationparticularly questions related to neural representations of valence or salience - cannot be addressed in the current work. Future studies should examine how these factors may affect reward processing in UD. Nevertheless, I highlight that the convergence of findings despite the heterogeneity of the included studies is striking and suggests that the current findings may reflect central abnormalities of UD.

Second, some included studies had relatively small sample sizes and reported coordinates that were not corrected for multiple comparisons, which may lead to biased results (Button et al., 2013). Future work should follow the most updated guidelines for best practices in the field to avoid generating biased findings (Nichols et al., 2017). Third, most of the included studies only recruited adults with acute major depression. More studies on other ages (e.g., pre-adolescents, adolescents) and mood states (e.g., remission) are needed. Fourth, I note that the search criteria were designed to focus on studies on reward and might not have identified some studies on punishment. Therefore, the analyses and results in relation to punishment are exploratory in nature and should be interpreted with caution. Fifth, the ALE method, by nature, cannot incorporate null results (Müller, Cieslik, Laird, et al., 2017). As a result, the current findings could be confounded by publication bias. Sixth, coordinate-based meta-analyses are blind to effect sizes. Therefore, I cannot make any claims about the magnitude of the effects observed in the current study. Finally, some patients in the included studies were medicated. The normalizing effects of treatment 
could obscure differences between UD and HC, increasing the probability of type II errors (Dichter et al., 2009).

Notwithstanding these caveats, the current meta-analysis shows that UD is consistently associated with opposing abnormalities in the reward circuit in response to reward: hypo-response in the VS and hyper-response in the OFC. The current metaanalytic results therefore suggest that UD is associated with both hypo and hyper responses to reward. UD may stem, in part, from dysregulated connectivity between the VS and the OFC. I believe the current findings will help lay a foundation towards developing a more refined understanding and treatment of UD and its comorbid psychiatric disorders, particularly ones that involve abnormal reward processing (Diehl et al., 2018). For example, a more refined understanding of the abnormalities in the reward circuitry in UD may help distinguish it from other disorders exhibiting reward processing abnormalities, such as bipolar disorder, schizophrenia, and substance use disorder. Moreover, although reinforcement learning models, such as actor-critic models and prediction error models, have been utilized to understand the pathophysiology of several psychiatric disorders (e.g., schizophrenia and addiction), research on their application on UD has been scant (Gold et al., 2012; Maia \& Frank, 2011). Future studies may consider how such models may be used to understand the pathophysiology of UD. The current results help delineate specific abnormalities within the reward circuit and supply a foundation for refining connectivity-based and computational models of UD. Finally, given that previous treatment targets for deep brain stimulation for treatment-resistant depression have yielded mixed results (Naesström et al., 2016), the portion of OFC implicated by the current results could be a promising treatment target. 


\section{CHAPTER 3}

\section{STUDY 2: AN FMRI STUDY OF REWARD PROCESSING DYSFUNCTION IN PREADOLESCENT MOOD DISORDERS}

In a sample of pre-adolescent children aged nine or ten recruited from 21 sites well-distributed across the United States (Garavan et al., 2018), Study 2 addressed two main aims. The first was to test whether pre-adolescent children with remitted BD or UD display abnormal patterns of neural activation and connectivity in response to reward, relative to $\mathrm{HC}$ without personal and family history of Axis I disorders. To date, most neuroimaging studies on reward processing dysfunction in mood disorders have focused on the depressive phase of the disorders. These studies, however, cannot address the question of whether reward processing abnormalities are stable characteristics of BD or UD and not simply consequences of depressive mood states. Indeed, one of the criteria for considering a characteristic as an endophenotype is state-independence, with the characteristic manifesting even outside of acute periods of illness (Gottesman \& Gould, 2003; Lenzenweger, 2013; G. A. Miller \& Rockstroh, 2013). In other words, in order to decide whether reward processing dysfunction is an endophenotype of BD or UD, it is necessary to examine whether it manifests even during remission. Taking into account a large volume of laboratory research documenting that behavioral abnormalities in reward processing persist into the remitted phase of BD and UD (Alloy et al., 2016), and prior research in adults showing increased OFC activity in BD or UD, increased striatal activity in BD, and decreased striatal activity in UD during reward processing (Study 1; Nusslock et al., 2012; Whitton et al., 2015), I hypothesized that OFC activity would be elevated in both remitted BD and remitted UD, but striatal activity would be increased in remitted $\mathrm{BD}$ and decreased in remitted UD during reward processing in pre-adolescents, 
relative to $\mathrm{HC}$. Based on research on connectivity in adults with mood disorders (Almeida et al., 2009; Felger et al., 2016; C. B. Young et al., 2016), I predict increased connectivity between the $\mathrm{OFC}$ and the striatum in response to reward in remitted $\mathrm{UD}$ and remitted $\mathrm{BD}$, relative to $\mathrm{HC}$.

To investigate the differences in neural responding to reward between $\mathrm{BD}$ and UD so as to enhance diagnostic accuracy, the second aim of this study was to compare remitted BD and remitted UD directly with each other to evaluate whether they can be distinguished by neural activation and connectivity during reward processing. Consistent with the reward hypersensitivity model of BD and reward hyposensitivity model of UD (Alloy et al., 2016), I hypothesized that remitted BD would show greater activity in the striatum and the OFC than remitted UD during reward processing. In light of prior research (Almeida et al., 2009), I also predicted that remitted BD would exhibit increased OFC-VS connectivity during reward processing, relative to remitted UD.

\section{Methods}

\section{Participants}

Participants were drawn from the first public data release of the Adolescent Brain Cognitive Development (ABCD) Study (Volkow et al., 2017). The data were collected between September 1, 2016 and September 15, 2017. All participants were aged nine or ten. Study design and recruitment procedures are described in detail in various publications (Casey et al., 2018; Garavan et al., 2018) and on the study's website (https://ABCDStudy.org). Briefly, the ABCD Study primarily recruited from a probability sampling of elementary schools located in 21 study sites distributed across the four major regions of the United States (i.e. Northeast, Midwest, South, and West). The catchment areas of the 21 sites include more than $20 \%$ of the entire 
population of 9- and 10-year-olds in the nation. Moreover, the demographics (e.g., race/ethnicity, sex) of potential participants living in the catchment areas of the study sites closely approximate those of the United States at large (Garavan et al., 2018). The ABCD Study also employed a variety of supplementary recruitment procedures, such as mailing lists, referrals, affiliates, summer recruitment, and twin registry, to recruit children who otherwise would not be reached through the standard schoolbased recruitment approach (e.g., home-schooled children). Research procedures of each site were governed by local, state, and federal regulations, overseen by local and/or centralized human subjects institutional review boards, and monitored by the ABCD External Advisory Board and the Bioethics and Medical Oversight advisory panel (Bjork, Straub, Provost, \& Neale, 2017; Clark et al., 2017). All participants underwent informed consent or assent procedures (Uban et al., 2018).

Participants only were included in the current study if they met the following inclusion criteria: (a) the presence of a T1 scan; (b) the presence of two scans from two runs of the Monetary Incentive Delay (MID) task, a monetary reward task, respectively (Knutson, Westdorp, Kaiser, \& Hommer, 2000; Yau et al., 2012); (c) the MID scans passed the ABCD Study's quality assurance metrics, including mean motion (average framewise displacement), the number of seconds with framewise displacements less than 0.2, 0.3, or $0.4 \mathrm{~mm}$ (Power, Barnes, Snyder, Schlaggar, \& Petersen, 2012), temporal signal-to-noise ratio, and temporal standard deviation (Triantafyllou et al., 2005); and (d) the scans were obtained from Siemens Prisma scanners, due to technical problems associated with scans obtained from General Electric 750 and Philips scanners. In addition to meeting data quality assurance criteria, to be included in the BD and UD groups of the current study, participants had to meet DSM-5 diagnostic criteria for remitted bipolar I disorder or remitted UD, 
according to either child- or parent-report on the Kiddie Schedule for Affective Disorders and Schizophrenia for DSM-5 (KSADS-5). To be included in the HC group, participants had to have no current or past DSM-5 Axis I psychopathology, according to both child- and parent-report on the KSADS-5, as well as no first- and seconddegree blood relatives with a history of psychopathology, according to parent report on a version of the Family History Assessment Module Screener (FHAM-S) (Rice et al., 1995). A total of 324 participants (95 BD, $73 \mathrm{UD}$, and $156 \mathrm{HC}$ ) met the above inclusion criteria. Fifty participants were randomly selected from each group for further analysis. After excluding 10 participants whose data had technical errors (e.g., acquisition errors), the final sample in the current study consisted of 140 participants (46 BD, $48 \mathrm{UD}$, and $46 \mathrm{HC}$ ). Out of the 46 participants with BD, 21 were diagnosed by parent-report only and 25 were by child-report only. Out of the 48 participants with UD, 31 were diagnosed by parent-report only; 16 were by child-report only; and 1 was by both child- and parent-report.

\section{Clinical Assessments}

Clinical assessments adopted in the ABCD Study are described comprehensively in Barch et al. (2017). In short, the self-administered computerized version of the KSADS-5 was used in the ABCD Study to assess current and past DSM-5 Axis I disorders in the pre-adolescent participants. It has been shown to have good-to-excellent kappas and high concordance with the clinicianadministered version of the KSADS-5 (Barch et al., 2017). Parents completed almost all of the modules in the KSADS-5, except for the enuresis, encopresis and selective mutism modules. Children also reported diagnostic information related to mood disorders, separation anxiety, social anxiety, sleep, and suicidality. Research assistants were trained to provide assistance to the children in completion of the KSADS-5. 
Family history of psychopathology was assessed using the family history method and a version of the FHAM-S. Parents gave information about the presence or absence of symptoms associated with alcohol use disorder, substance use disorder, depression, mania, psychosis, and antisocial personality disorder in all first- and second-degree biological relatives of the children, which include full and halfsiblings, parents, grandparents, aunts, and uncles. The family history method was considered reasonable and adequate for the purpose of the study, given that the family study method, where relatives are interviewed directly, would have been impractical (Andreasen, Rice, Endicott, Reich, \& Coryell, 1986; Barch et al., 2017; Rice et al., 1995).

\section{Potential Covariates}

As handedness, medication usage, trait reward sensitivity, and concurrent mood symptoms may influence brain activity, a variety of self-report measures were used to examine any potential correlations of these variables with brain activation and connectivity and determine whether they should be included as covariates in subsequent analyses. First, handedness was assessed using the four-item Youth Edinburgh Handedness Inventory Short Form (Oldfield, 1971; Veale, 2014). Participants were asked to rate on a five-point scale ("always right hand" to "always left hand") the hand they typically use for writing, throwing, using a spoon, and using a toothbrush. A laterality quotient was calculated to reflect the degree to which participants were right-handed, left-handed, or ambidextrous. Second, medication usage in the past two weeks was evaluated using the Medication Inventory from the PhenX Toolkit October 1, 2015 Ver. 13.0 (Barch et al., 2017; Bild et al., 2002). Parents were asked to bring all of their children's prescriptions to the in-person assessment and answer questions about usage of prescribed and over-the-counter 
medications. Third, trait reward sensitivity was assessed using an abridged version of the BIS/BAS scales (Carver \& White, 1994; Pagliaccio et al., 2016), which consist of a subscale to assess the Behavioral Inhibition System and three subscales to measure the Behavioral Approach System (BAS; Reward Responsiveness, Drive, and Fun Seeking). The three BAS subscales were used in the current study to quantify trait reward sensitivity, and they have demonstrated acceptable internal consistencies in the ABCD sample, with alphas: BAS Drive (.78), BAS Reward Responsiveness (.62), and BAS Fun Seeking (.64) (Barch et al., 2017). Finally, concurrent depressive and manic symptoms were assessed using the well-validated DSM-5 Depression subscale of the Child Behavior Checklist (Achenbach, 2009) and a mania scale from the Parent General Behavior Inventory (Youngstrom, Frazier, Demeter, Calabrese, \& Findling, 2008), respectively.

\section{MID Task}

The MID task was administered during fMRI to probe neural responses to the anticipation and receipt of rewards and losses. On each trial of the MID task, participants were first presented with an incentive cue $(2000 \mathrm{~ms})$ signaling that they had a chance to (a) win $\$ .20$ (small reward), (b) win $\$ 5$ (large reward), (c) lose $\$ .20$ (small loss), (d) lose \$5 (large loss), or (e) not lose or win any money (neutral). These five possibilities represented the five trial types of the MID task. After the incentive cue, an anticipation period ensued (1500-4000 ms). Next, the participant responded to a variable target (150-500 ms). Finally, participants were shown feedback on the outcome of the trial. The feedback was displayed for $2000 \mathrm{~ms}$ minus the target duration. Twelve optimized trial orders were developed for the study (two runs each). Each of the two runs involved 50 trials (10 per trial type) presented in pseudorandom order. Task difficulty was adjusted after every third incentivized trial on the basis of 
the overall accuracy rate of the last six trials, so that each participant achieved an accuracy rate of approximately $60 \%$. Each participant was presented with 40 reward and loss anticipation trials and 20 no money anticipation trials; 24 positive feedback trials (for both reward and loss) and 16 negative feedback trials (for both reward and loss), on average, were generated by the adaptive algorithm. Preliminary tests of the ABCD Study MID data showed that the average hit rate is higher for the reward (59\%) and loss (54\%) trials than for neutral trials (49\%), and that the VS and medial PFC were robustly activated in response to reward (Casey et al., 2018). More details about the validity of the data derived from the MID task used in the ABCD Study are described in Casey et al. (2018).

\section{Image Acquisition}

ABCD's imaging data were collected using 28 3T scanners from three different platforms (Siemens Prisma, General Electric 750, and Philips) at the 21 participating sites (Casey et al., 2018; Nielson et al., 2018). The ABCD imaging protocol was harmonized to be compatible across the platforms (Casey et al., 2018). High spatial and temporal resolution fMRI images were acquired [matrix, 90 x 90; slices, 60; field of view (FOV), 216 x 216; \% FOV phase, 100\%; resolution, 2.4 x 2.4 x $2.4 \mathrm{~mm}$; repetition time (TR), $800 \mathrm{~ms}$; echo time (TE), $30 \mathrm{~ms}$; flip angle, 52 degree; multiband acceleration, 6]. 3D T1 and 3D T2 weighted images [matrix, $256 \times 256$; resolution, 1.0 × $1.0 \times 1.0 \mathrm{~mm}$; inversion time (TI), $1060 \mathrm{~ms}$ ] also were acquired. All images were acquired in the axial plane. More details about the imaging parameters are described in Casey et al. (2018).

\section{Image Preprocessing}

Imaging data used in the current study were organized in accordance with the Brain Imaging Data Structure format (Gorgolewski et al., 2016) using HeuDiConv 
(https://github.com/nipy/heudiconv), and were pre-processed using FMRIPREP v1.3.0.post2 (Esteban et al., 2018). T1-weighted volumes were corrected for intensity non-uniformity using N4BiasFieldCorrection v2.1.0 (Tustison et al., 2010), skullstripped using antsBrainExtraction.sh v2.1.0 and the OASIS template, and coregistered to skull-stripped ICBM 152 Nonlinear Asymmetrical template version 2009c (Fonov, Evans, McKinstry, Almli, \& Collins, 2009) via nonlinear registration using the antsRegistration of ANTs v2.1.0 (Avants, Epstein, Grossman, \& Gee, 2008). Brain tissue segmentation of cerebrospinal fluid, white matter, and gray matter on the brain-extracted T1-weighted volumes were conducted using FAST of FSL v5.0.9 (Y. Zhang, Brady, \& Smith, 2001).

Functional data were motion corrected using MCFLIRT of FSL v5.0.9 (Jenkinson, Bannister, Brady, \& Smith, 2002) and spatially normalized to a standard stereotactic space as defined by the Montreal Neurological Institute (MNI). FLIRT of FSL v5.0.9 was used for co-registration to the corresponding T1-weighted volume using boundary-based registration (Greve \& Fischl, 2009) with 9 degrees of freedom. Motion correcting transformations, BOLD-to-T1-weighted transformation, and T1-weighted-to-template (MNI) warp were concatenated and applied using antsApplyTransforms of ANTs v2.1.0 with Lanczos interpolation. Functional images were spatially smoothed with a $4.8 \mathrm{~mm}$ full-width-half-maximum Gaussian kernel and subjected to a high-pass temporal filter with a 100s cutoff. Additionally, ICA-based Automatic Removal Of Motion Artifacts (AROMA) were used to reduce motion-induced signal variations in data (Pruim et al., 2015). More details of FMRIPREP can be found on its website (https://fmriprep.readthedocs.io/en/latest/workflows.html). 
Neuroimaging analyses were conducted using FSL v6.0.0. A general linear model (GLM) with autocorrelation correction was adopted. First, a first-level model was constructed for each participant with a total of 15 regressors reflecting anticipation and feedback (positive and negative) of the five trial types: (a) win $\$ .20$ (small reward), (b) win \$5 (large reward), (c) lose \$.20 (small loss), (d) lose \$5 (large loss), or (e) not lose or win any money (neutral). The 15 regressors were convolved with the canonical hemodynamic response function. At the second-level analyses, data from the two runs of the MID task were combined for each participant using a fixed effects model. For third-level analyses (i.e., across participants), group statistical maps for reward (\$.20 and $\$ 5.0$ combined) and loss (\$.20 and $\$ 5.0$ combined) versus neutral incentive (\$0) during anticipation and feedback (positive and negative) were calculated using one-sample $t$ tests.

The five trial types of the MID task allowed for the examination of valence and salience signals during reward processing (Figure 5). Specifically, on the one hand, most theorists have suggested that brain activation during reward processing reflects the valence or value of a stimulus and follows a linear pattern (Bartra et al., 2013; Cooper \& Knutson, 2008), with higher activation reflecting higher desirability and positive valence or value of a stimulus. On the other hand, some have identified that brain activity during reward processing also at times may represent the salience of a stimulus and follow a U-shaped pattern, with higher activation reflecting higher importance and absolute value of a stimulus. Given that reward-related brain activation can be driven by the valence and/or salience of stimuli (Bartra et al., 2013; Kahnt, Park, Haynes, \& Tobler, 2014; Litt, Plassmann, Shiv, \& Rangel, 2011; Zink, Pagnoni, Chappelow, Martin-Skurski, \& Berns, 2006), both valence and salience 
signals were examined for anticipation. Only valence signals were examined for feedback due to technical errors in the ABCD data.
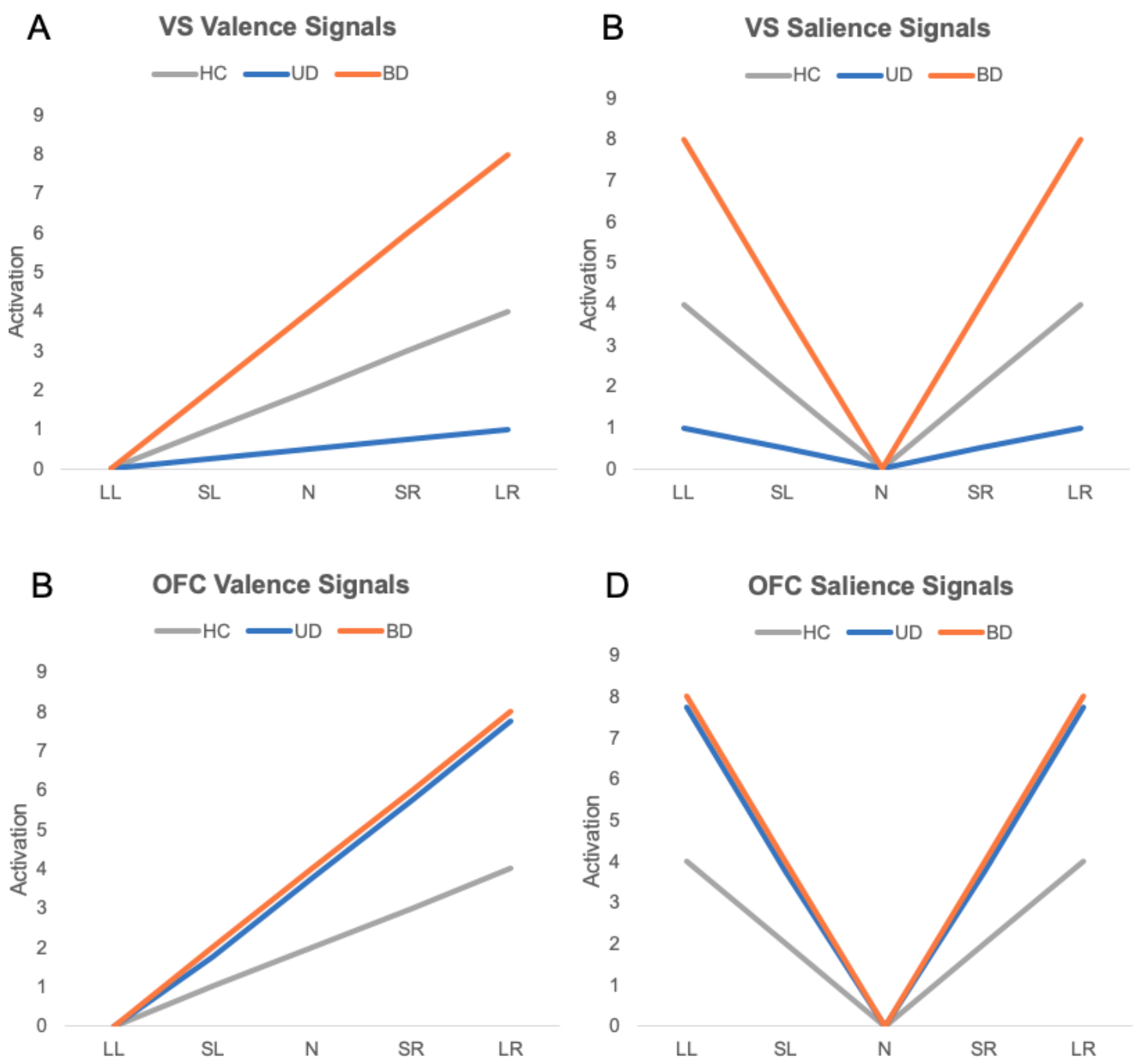

Figure 5. Illustration of valence and salience signals based on study hypotheses. $\mathrm{LL}=$ large loss; $\mathrm{SL}=$ small loss; $\mathrm{N}=$ neutral; $\mathrm{SR}=$ small reward $\mathrm{LR}=$ large reward. $\mathrm{I}$ hypothesized that during reward processing, VS activity would be increased in remitted $\mathrm{BD}$ and decreased in remitted UD, but OFC activity would be increased in both remitted $\mathrm{BD}$ and remitted UD, relative to $\mathrm{HC}$. I also hypothesized that remitted BD would show greater activity in the VS and the OFC than remitted UD during reward processing. (A) and (B) demonstrate expected results if activation in the VS and OFC reflects the valence of a stimulus and follows a linear pattern, with higher activation reflecting higher desirability and positive valence of a stimulus. (C) and (D) 
demonstrate expected results if activation in the VS and OFC represents the salience of a stimulus and follow a U-shaped pattern, with higher activation reflecting higher importance and absolute value of a stimulus.

Based on my a priori hypotheses of differences in the OFC and the VS between the groups, I conducted ROI analyses involving activation in these regions. Anatomical masks generated by Study 1 (Figure 3) were used for OFC and VS. Average time course of reward-related activity in the ROIs were extracted using FSL's fslmeants. Specifically, Group x Condition mixed ANCOVAs and one-way ANCOVAs were performed for the ROI analyses. Greenhouse-Geisser correction was utilized for violations of sphericity. Post-hoc two-sample $t$ tests were conducted to probe the nature of any significant interactions. Whole-brain analyses were conducted to explore between-group differences outside the ROIs.

To evaluate connectivity between the OFC and the VS, PPI analyses (Friston et al., 1997; McLaren et al., 2012), a technique that examines whether a psychological context (e.g., reward processing) changes how one brain region (a "seed region") contributes to another brain region (a "target region") by testing whether a significant interaction between the psychological context and the seed region is shown in the target region, were conducted. This approach has been shown to reveal consistent and specific patterns of connectivity across studies (D. V. Smith, Gseir, et al., 2016). The first-level PPI models mostly involved the same task regressors described above, with the addition of a physiological regressor representing the time course of rewardrelated activation within the ROIs, and interaction terms between the physiological regressor and the task regressors. At the second-level analyses, data from the two runs of the MID task were combined for each participant using a fixed effects model. 
Group differences in connectivity during reward processing were examined in thirdlevel analyses.

To determine if there were differences in reward-related neural responses between $\mathrm{BD}, \mathrm{UD}$, and $\mathrm{HC}$ on the network level, five a priori generated corticostriatal network maps (CSNmaps) identified by Dr. David Smith using tensorial independent component analysis (ICA; Beckmann \& Smith, 2005) on the neuroimaging data of the reward consumption task (Delgado et al., 2000) from 20 participants in the Human Connectome Project (Van Essen et al., 2013) were used. Created based on a novel connectivity-based framework that aims to fully capture the complexity of rewardrelated brain activation by focusing on the whole brain rather than single regions, the five CSNmaps are five whole-brain tensor components whose temporal fluctuations corresponded to the presentation of rewards and punishments and involved the striatum (Figure 6). They represent five distinct patterns of corticostriatal connectivity involved in reward processing, with some exhibiting positive connectivity between the dorsal striatum and dIPFC (e.g., IC 1) and others showing negative connectivity between the VS and fronto-parietal network (e.g., IC 7). To examine group differences that might be reflected in the whole brain rather than single regions, oneway ANCOVAs were performed to compare whether there were differences between $\mathrm{BD}, \mathrm{UD}$, and $\mathrm{HC}$ in their spatial correlations between the five CSNmaps and the whole-brain reward responses during each condition for each participant, with higher correlations representing higher similarity between their whole-brain reward responses and the whole-brain reward responses from healthy individuals in HCP. 
CSNmaps derived from Tensor-based ICA

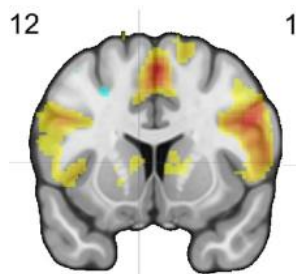

IC 1

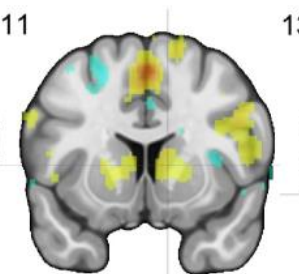

IC 2

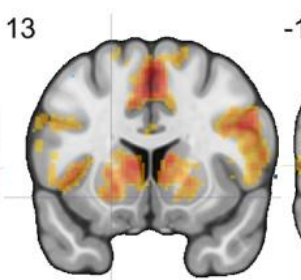

IC 3

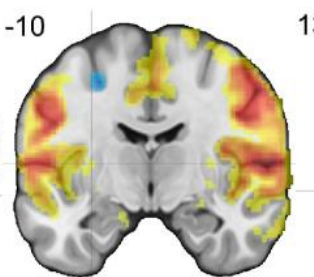

IC 5

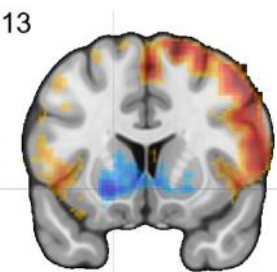

IC 7

Figure 6. CSNmaps derived from tensorial independent component analysis. The five CSNmaps were derived from 20 participants performing the reward consumption in the Human Connectome Project. The colors represent positive (red) and negative (blue) loadings onto that component. The figure was created by Dr. David Smith.

The relationships between brain activation, connectivity, network patterns and potential confounders (age, sex, race, handedness, reward sensitivity, medication use, and concurrent mood symptoms) were examined, and where appropriate, such variables also were included as covariates in all analyses. All z-statistic images were thresholded and corrected for multiple comparisons using an initial cluster-forming threshold of uncorrected $p<.001$, followed by a whole-brain corrected cluster-extent threshold of $p<0.05$, as determined using nonparametric permutation tests, which do not assume Gaussian distributions (Eklund, Nichols, \& Knutsson, 2016).

Results

\section{Participant Characteristics}

Demographic and clinical characteristics of the participants in the three groups were compared using one-way ANOVAs and chi-square tests (Table 7). There was a significant main effect of group on depressive symptoms, $F(2,137)=17.10, p<.01$. Tukey's post-hoc tests revealed that HC reported significantly lower depressive symptoms compared to BD (mean difference $=-2.72, S E=0.63, p<.01)$ and UD 
(mean difference $=-3.45, S E=0.62, p<.01)$ groups. There was no significant difference between BD and UD (mean difference $=0.73, S E=0.62, p=.47$ ) in depressive symptoms. In addition, there was a significant main effect of group on hypomanic symptoms, $F(2,137)=6.37, p<.01$. Tukey's post-hoc tests suggested that $\mathrm{HC}$ reported significantly fewer hypomanic symptoms than BD (mean difference $=-2.35, S E=0.80, p<.05)$ and UD (mean difference $=-2.58, S E=0.80, p<.01$ ). There was no significant difference between BD and UD (mean difference $=0.23, S E$ $=0.80, p=.95)$. In addition, a chi-square test identified a statistically significant association between group and medication usage in the past two weeks, $\chi^{2}(2, N=140)$ $=12.63, p<.01(\mathrm{BD}$ medication usage $=45.7 \% ; \mathrm{UD}=54.2 \% ; \mathrm{HC}=19.6 \%) . \mathrm{BD}$, UD, and HC did not differ significantly with respect to age, sex, race, handedness, BAS Drive, BAS Fun Seeking, and BAS Reward Responsiveness (all $p$ 's $>.05$ ). 
Table 7

Characteristics of Participants in Study 2

\begin{tabular}{|c|c|c|c|c|}
\hline & $\begin{array}{l}\text { Overall } \\
(n=140)\end{array}$ & $\begin{array}{l}\mathrm{BD} \\
(n=46)\end{array}$ & $\begin{array}{l}\text { UD } \\
(n=48)\end{array}$ & $\begin{array}{l}\mathrm{HC} \\
(n=46)\end{array}$ \\
\hline $\begin{array}{l}\text { Age, months } \\
(S D)\end{array}$ & $121.38(6.77)$ & $121.91(6.50)$ & $121.40(6.73)$ & $120.83(7.17)$ \\
\hline $\begin{array}{l}\text { Female } \\
\text { Race }\end{array}$ & $49.3 \%$ & $45.7 \%$ & $47.9 \%$ & $54.3 \%$ \\
\hline $\begin{array}{l}\text { American } \\
\text { Indian/ } \\
\text { Alaska } \\
\text { Native }\end{array}$ & $0.7 \%$ & $2.2 \%$ & $0 \%$ & $0 \%$ \\
\hline Asian & $2.1 \%$ & $0 \%$ & $2.1 \%$ & $4.3 \%$ \\
\hline $\begin{array}{l}\text { Black or } \\
\text { African } \\
\text { American }\end{array}$ & $10.7 \%$ & $15.2 \%$ & $8.3 \%$ & $8.7 \%$ \\
\hline White & $72.1 \%$ & $69.6 \%$ & $77.1 \%$ & $69.6 \%$ \\
\hline $\begin{array}{l}\text { More Than } \\
\text { One Race }\end{array}$ & $9.3 \%$ & $8.7 \%$ & $8.3 \%$ & $10.9 \%$ \\
\hline Other & $4.3 \%$ & $4.3 \%$ & $2.1 \%$ & $6.5 \%$ \\
\hline $\begin{array}{l}\text { Do Not } \\
\text { Know }\end{array}$ & $0.7 \%$ & $0 \%$ & $2.1 \%$ & $0 \%$ \\
\hline $\begin{array}{l}\text { Hispanic } \\
\text { EHIS } \\
\text { Handedness }\end{array}$ & $29.3 \%$ & $23.9 \%$ & $25.0 \%$ & $39.1 \%$ \\
\hline $\begin{array}{l}\text { Right- } \\
\text { Handed }\end{array}$ & $77.9 \%$ & $73.9 \%$ & $75.0 \%$ & $84.8 \%$ \\
\hline Left-Handed & $12.1 \%$ & $13.0 \%$ & $18.8 \%$ & $4.3 \%$ \\
\hline Mixed & $10.0 \%$ & $13.0 \%$ & $6.3 \%$ & $10.9 \%$ \\
\hline $\begin{array}{l}\text { Medication } \\
\text { Use }\end{array}$ & $40.0 \%$ & $45.7 \%$ & $54.2 \%$ & $19.6 \%$ \\
\hline $\begin{array}{l}\text { CBCL } \\
\text { Depression } \\
(S D)\end{array}$ & $2.40(3.33)$ & $3.04(3.70)$ & $3.77(3.48)$ & $.33(.99)$ \\
\hline $\begin{array}{l}\text { PGBI Mania } \\
(S D)\end{array}$ & $2.22(4.00)$ & $2.91(4.38)$ & $3.15(4.82)$ & $.57(1.34)$ \\
\hline $\begin{array}{l}\text { BAS Drive } \\
(S D)\end{array}$ & $4.34(3.11)$ & $4.09(2.65)$ & $4.42(3.48)$ & $4.52(3.17)$ \\
\hline $\begin{array}{l}\text { BAS Fun } \\
\text { Seeking }(S D)\end{array}$ & $6.36(2.68)$ & $6.24(2.51)$ & $6.44(2.95)$ & $6.41(2.61)$ \\
\hline $\begin{array}{l}\text { BAS Reward } \\
\text { Responsivenes } \\
\text { s }(S D)\end{array}$ & $11.14(2.78)$ & $10.98(2.86)$ & $11.08(2.77)$ & $11.37(2.74)$ \\
\hline
\end{tabular}

Note. $\mathrm{BD}=$ Bipolar Disorder; $\mathrm{UD}=$ Unipolar Depression; $\mathrm{HC}=$ Healthy Controls;

EHIS $=$ Youth Edinburgh Handedness Inventory Short Form; CBCL $=$ Child 
Behavior Check List; PGBI = Parent General Behavior Inventory; BAS = Behavioral Approach System.

\section{Associations Among Study Variables}

Pearson correlations were conducted to examine the associations of continuous demographic and clinical variables (age, mood symptoms, and reward sensitivity) with activation in the OFC and the VS and OFC-VS functional connectivity as well as connectivity during reward anticipation and feedback (Table 8). Results indicated that age was negatively associated with OFC activation during anticipation (valence) across the whole sample, $r(138)=-.19, p<.05$, such that older participants had lower OFC activation during anticipation (valence). Additional analyses revealed that the significant association was found only in $\mathrm{BD}, r(44)=-.31$, $p<.05$, but not in UD, $r(46)=-.07, p=.65$, and HC, $r(44)=-.20, p=.19$. Additionally, BAS Drive was negatively associated with OFC activation during anticipation (salience), $r(138)=-.19, p<.05$, and VS activation during anticipation (salience) across the whole sample, $r(138)=-.26, p<.01$, such that participants with higher BAS Drive scores had lower OFC and VS activation during anticipation (salience). Additional analyses revealed that the significant association was found only in $\mathrm{HC}, r(44)=-.39, p<.01$, but not in UD, $r(46)=-.06, p=.67$, and $\mathrm{BD}, r(44)=$ $-.20, p=.19$. Furthermore, BAS Reward Responsiveness was negatively correlated with VS activation during feedback, $r(138)=-.17, p<.05$, such that participants with higher BAS Reward Responsiveness scores had lower VS activation during feedback. Additional analyses revealed that the significant association was not found in BD, $r(44)=-.19, p=.20, \mathrm{UD}, r(46)=-.03, p=.84$, and HC, $r(44)=-.28, p=.06$. Finally, OFC-VS connectivity during anticipation (salience) was negatively associated with 
hypomanic symptoms, such that participants with higher connectivity between OFC and VS had lower hypomanic symptoms, $r(138)=-.21, p<.05$. Additional analyses revealed that the significant association was not found in $\mathrm{BD}, r(44)=-.28, p=.06$, $\mathrm{UD}, r(46)=-.26, p=.07$, and $\mathrm{HC}, r(44)=-.01, p=.97$.

Two-sample $t$-tests and one-way ANOVAs were conducted to examine the associations of categorical demographic and clinical variables (gender, race, handedness, and medication use) with activation in the OFC and the VS as well as OFC-VS functional connectivity and connectivity between them during anticipation and feedback. Results suggested that there was a significant effect for gender, $t(138)=$ $2.46, p<.05$, such that females had greater activation in the VS during anticipation (valence) than males. Moreover, there was a significant main effect of handedness on OFC-VS connectivity during anticipation (valence), $F(2,137)=4.32, p<.05$. Tukey's post-hoc tests revealed that mixed-handed individuals had less connectivity between the OFC and VS during anticipation (valence) compared to right-handed (mean difference $=-0.32, S E=0.12, p<.05$ ) and left-handed individuals (mean difference $=-0.42, S E=0.15, p<.05)$. There were no significant associations between the ROI variables and race or medication usage (all $p$ 's $>.05$ ). 
Table 8

Pearson Correlations between Regions of Interest and Participant Characteristics

\begin{tabular}{|c|c|c|c|c|c|c|}
\hline & Age & Hyp & Dep & Drive & FS & RR \\
\hline \multicolumn{7}{|l|}{ Activation During Anticipation } \\
\hline OFC (Valence) & $-.19 *$ & .07 & .06 & .14 & -.04 & .02 \\
\hline OFC (Salience) & -.07 & -.01 & .01 & $-.19 *$ & -.09 & -.07 \\
\hline VS (Valence) & -.13 & -.10 & -.06 & -.02 & -.13 & -.09 \\
\hline VS (Salience) & -.07 & -.06 & -.04 & $-.26 * *$ & -.15 & -.16 \\
\hline \multicolumn{7}{|l|}{ Activation During Feedback } \\
\hline $\mathrm{OFC}$ & -.05 & -.11 & .07 & .08 & .02 & -.09 \\
\hline VS & -.15 & -.01 & .16 & .08 & .03 & $-.17 *$ \\
\hline \multicolumn{7}{|l|}{ Connectivity During Anticipation } \\
\hline OFC-VS (Valence) & .02 & .16 & .15 & -.05 & .00 & .02 \\
\hline OFC-VS (Salience) & .04 & $-.21 *$ & -.10 & -.04 & .02 & -.09 \\
\hline $\begin{array}{l}\text { OFC-VS Connectivity During } \\
\text { Feedback }\end{array}$ & -.04 & .00 & .02 & .05 & -.04 & .04 \\
\hline OFC-VS Functional Connectivity & .00 & -.04 & -.04 & -.09 & -.04 & .02 \\
\hline
\end{tabular}

Note. Hyp = Hypomanic Symptoms Assessed by Parent General Behavior Inventory -

Mania; Dep = Depressive Symptoms Assessed by Child Behavior Check List's DSM-

5 Depression Subscale Raw Scores; Drive = Drive Subscale of the BIS/BAS Scales;

FS = Fun Seeking Subscale of the BIS/BAS Scales; RR = Reward Responsiveness

Subscale of the BIS/BAS Scales. ${ }^{*} p<.05 ;{ }^{*} p<.01$.

\section{Behavioral Results}

\section{Reaction Time}

A two-way ANOVA of reaction times was conducted for positive feedback trials, with condition (large loss, small loss, neutral, small reward, large reward) as the within-subject factor and group (BD, UD, HC) as the between-subjects factor. There were no statistically significant interactions between group and condition for reaction times, $F(7.300,430.678)=0.549, p=.804($ Figure 7$)$.

One-way ANOVAs revealed no main effect of group on reaction times, $F(2$, $118)=1.616, p=.203$, but there was a significant main effect of condition, $F(3.654$, $438.431)=5.710, p<.01$. Bonferroni-adjusted post-hoc tests suggested that 
participants exhibited greater reaction times during the neutral condition than the large loss condition (mean difference $=6.770, S E=1.865, p<.01$ ) and the large reward condition (mean difference $=5.923, S E=1.725, p<.01$ ). They also exhibited higher reaction times during the small reward condition than the large loss condition (mean difference $=4.297, S E=1.449, p<.05)$.

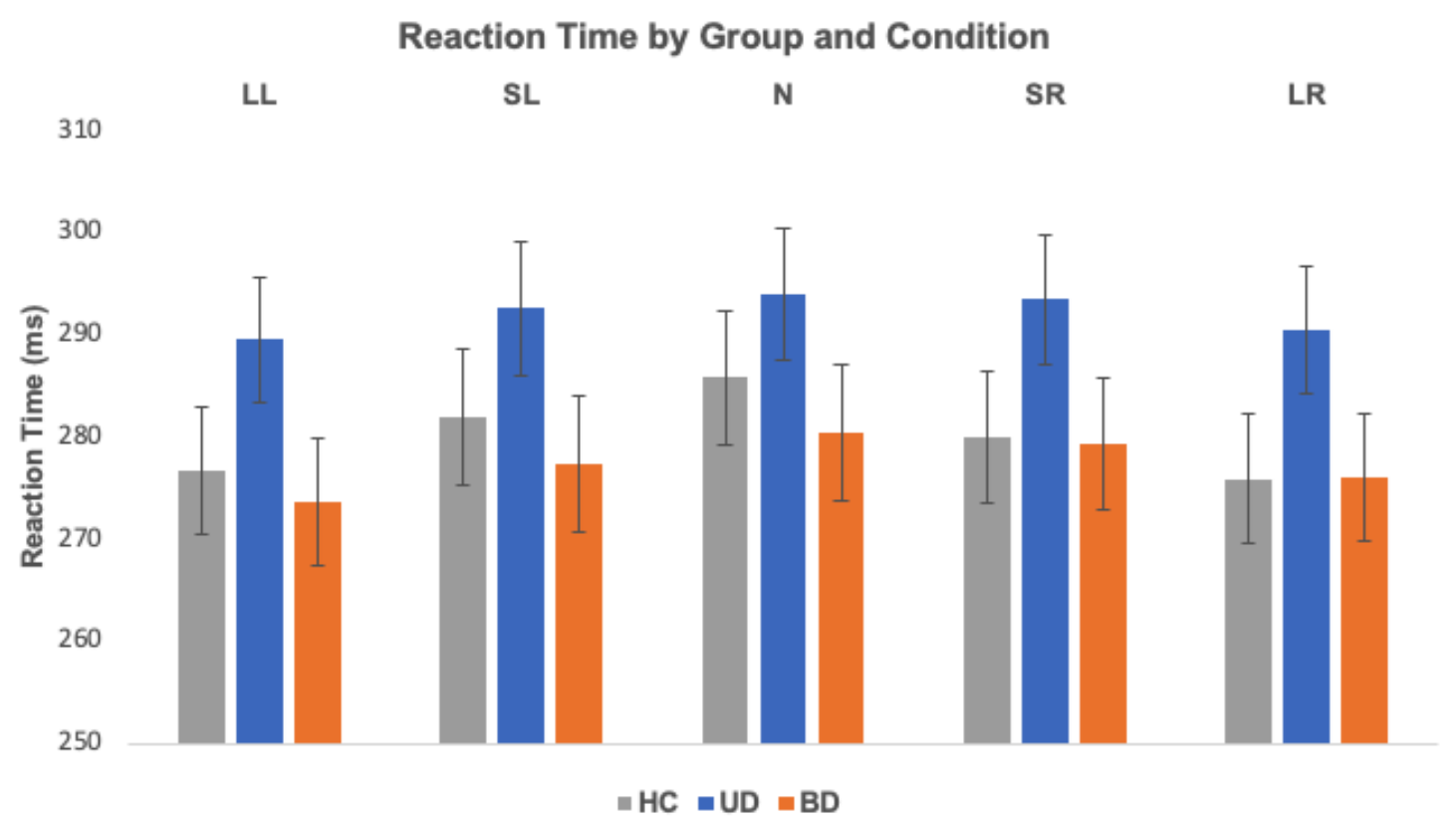

Figure 7. Reaction time by group and condition during the MID task.

\section{Hit Rate}

A Group X Condition ANOVA of hit rates was conducted for positive feedback trials. There was a statistically significant interaction between group and condition for hit rates, $F(7.387,435.857)=2.033, p<.05$ (Figure 8$)$. Post-hoc analyses suggested that UD had higher hit rates during the neutral condition than BD (mean difference $=0.069, S E=0.280, p<.05)$ and $\mathrm{HC}($ mean difference $=0.067, S E$ $=0.280, p<.05)$ after Tukey's HSD adjustment, but there were no differences between $\mathrm{BD}$, UD, and $\mathrm{HC}$ during the large loss condition, $F(2,118)=1.335, p=.267$, 
the small loss condition, $F(2,118)=1.410, p=.248$, the small reward condition, $F(2$, $118)=0.368, p=.693$, and the large reward condition, $F(2,118)=0.509, p=.602$.

One-way ANOVAs revealed no main effect of group on hit rates, $F(2,118)=$ $0.345, p=.709$, but there was a significant main effect of condition, $F(3.742$, $448.983)=26.353, p<.01$. Bonferroni-adjusted post-hoc tests suggested that participants exhibited lower hit rates during the neutral condition than the large loss condition (mean difference $=-0.120, S E=0.015, p<.01)$, the small loss condition (mean difference $=-0.067, S E=0.014, p<.01)$, the small reward condition (mean difference $=-0.069, S E=0.014, p<.01)$, and the large reward condition (mean difference $=-0.131, S E=0.016, p<.01)$. They also exhibited lower hit rates during the small loss condition than the large loss condition (mean difference $=-0.054, S E=$ $0.015, p<.01$ ) and the large reward condition (mean difference $=-0.064, S E=0.016$, $p<.01)$. Finally, they showed lower hit rates during the small reward condition than the large loss condition (mean difference $=-0.052, S E=0.014, p<.01$ ) and the large reward condition (mean difference $=-0.062, S E=0.014, p<.01)$.

\section{Hit Rate by Group and Condition}

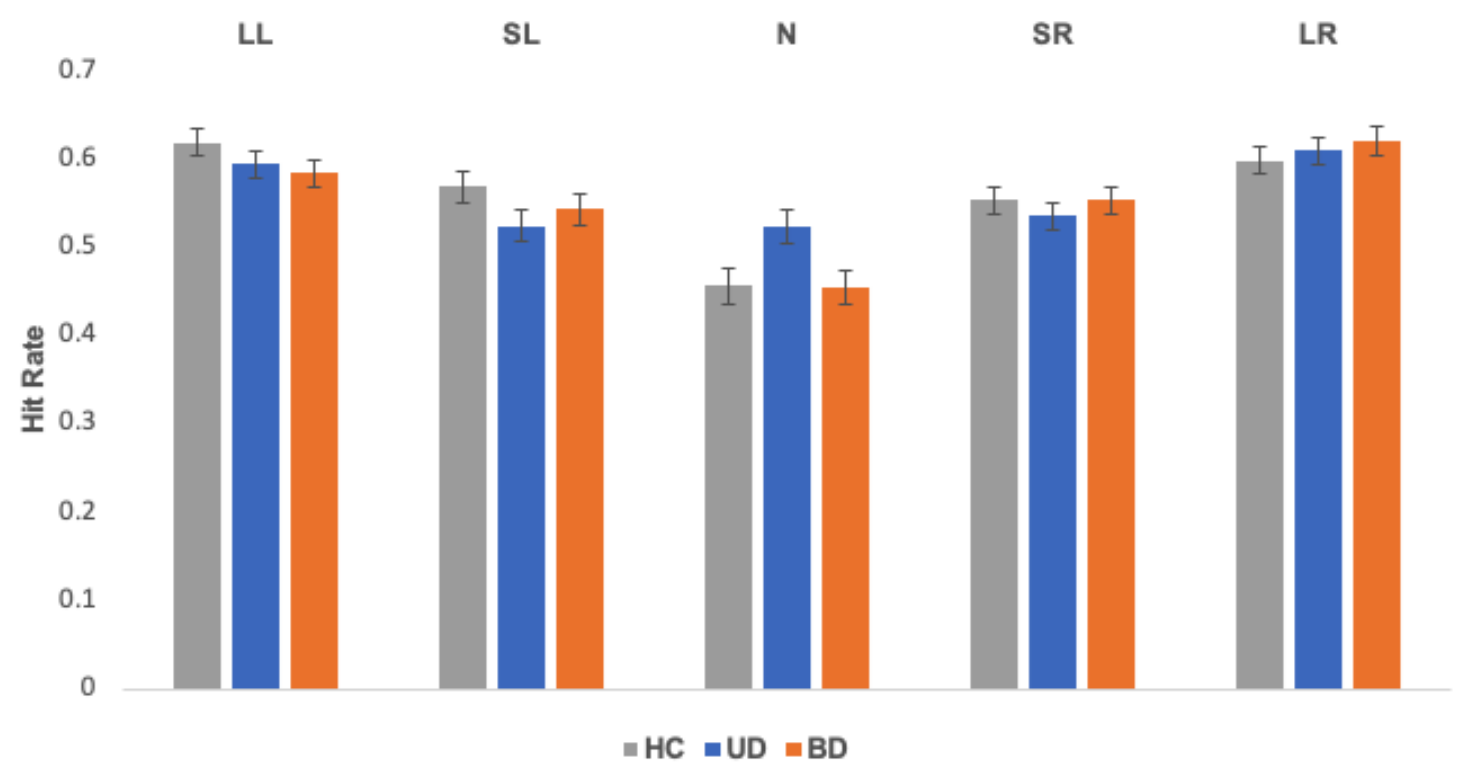

Figure 8 . Hit rate by group and condition during the MID task. 


\section{Neuroimaging Results}

To address the major aims of this study, a series of one-way ANCOVAs were conducted to examine whether there were significant differences between $\mathrm{BD}, \mathrm{UD}$, and $\mathrm{HC}$ in reward-related neural activation, connectivity, and networks. If there was a significant difference, a two-way mixed ANCOVA was conducted to explore what contributed to the effect. Given that age, gender, handedness, and hypomanic symptoms were associated with at least one ROI variable, they were included as covariates in all ROI analyses. Whole-brain analyses were conducted to explore between-group differences outside the ROIs.

\section{Activation Analyses}

First, two ANCOVAs were run to examine the effect of group on OFC activation during anticipation (valence and salience; Figure 9), after controlling for age, gender, handedness, and hypomanic symptoms. Levene's tests of homogeneity of variance indicated that there was homogeneity of variances ( $p$ 's > .05). Results indicated that there were no statistically significant differences between BD, UD, and $\mathrm{HC}$ in OFC activation during anticipation (valence), $F(2,133)=1.193, p=.307$, partial $\eta^{2}=.018$ (Figure 9A), and anticipation (salience), $F(2,133)=0.094, p=.911$, partial $\eta^{2}=.001$ (Figure 9B). Group differences in OFC activation by reward anticipation condition were plotted for interpretative purposes (Figure 9C). 


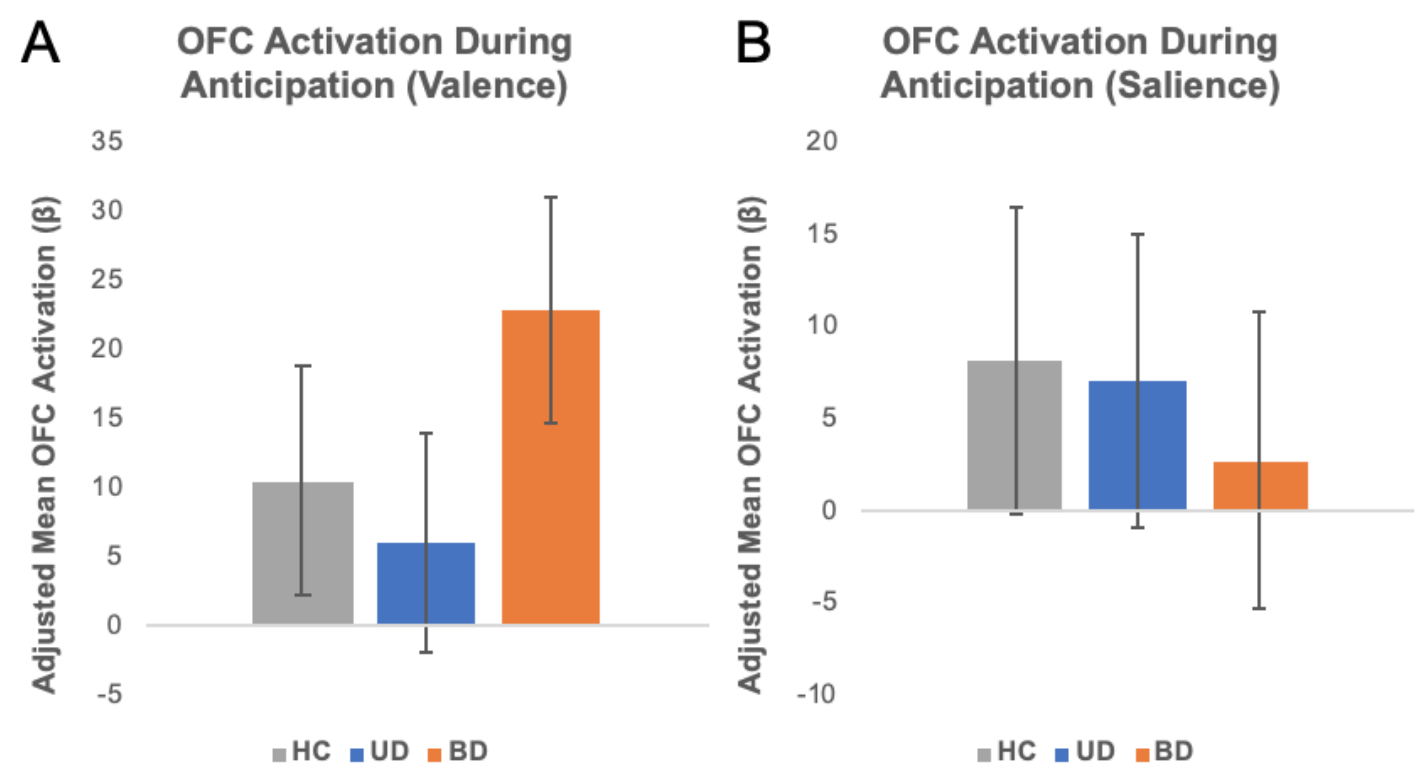

C OFC Activation by Group and Reward Anticipation Condition

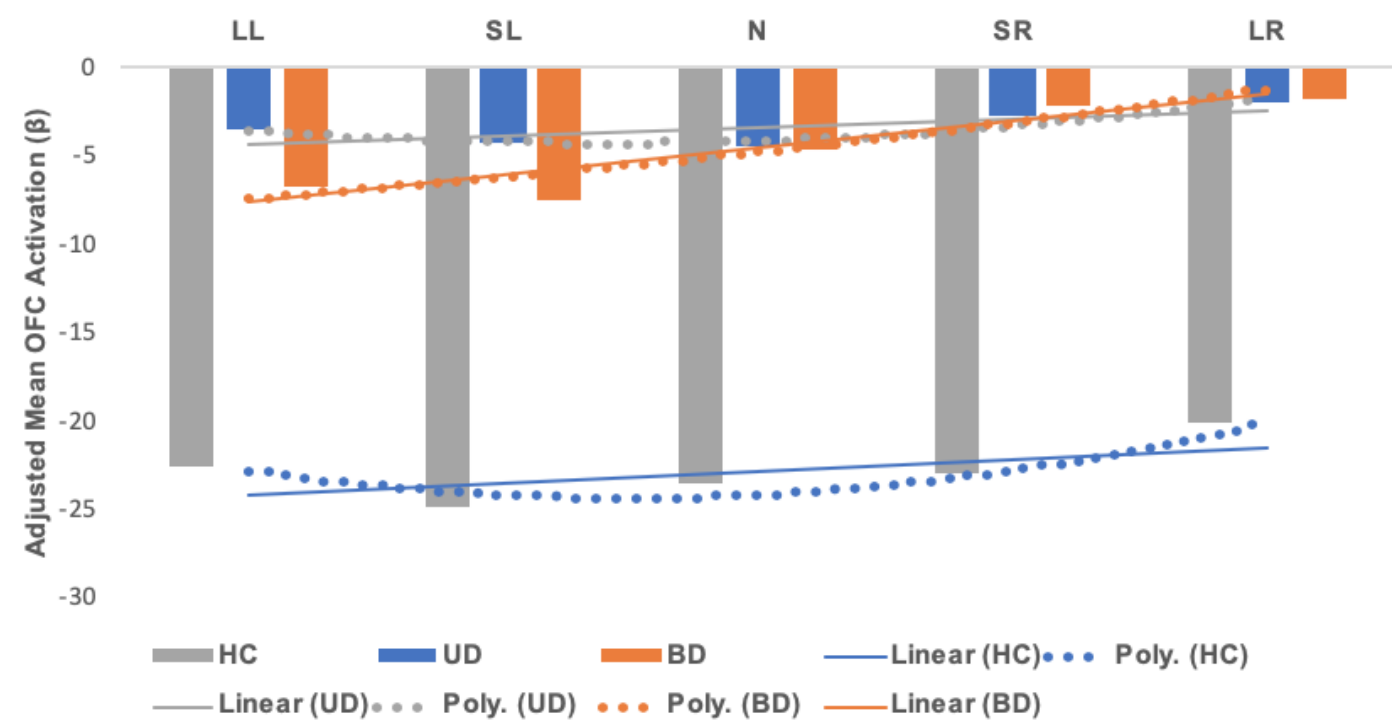

Figure 9. Group differences in OFC activation during anticipation. Age, gender, handedness, and hypomanic symptoms were adjusted in the analyses.

Second, two additional ANCOVAs were run to examine the effect of group on VS activation during anticipation (valence and salience; Figure 10), after controlling for age, gender, handedness, and hypomanic symptoms. Levene's tests of homogeneity of variance indicated that there was homogeneity of variances ( $p$ 's > $.05)$. Results indicated that there were no statistically significant differences between 
$\mathrm{BD}, \mathrm{UD}$, and $\mathrm{HC}$ in VS activation during anticipation (valence), $F(2,133)=$

$0.911, p=.405$, partial $\eta^{2}=.014$ (Figure 10A), and anticipation (salience), $F(2,133$ )

$=0.568, p=.568$, partial $\eta^{2}=.008$ (Figure 10B). Group differences in VS activation by reward anticipation condition were plotted for interpretative purposes (Figure 10C).
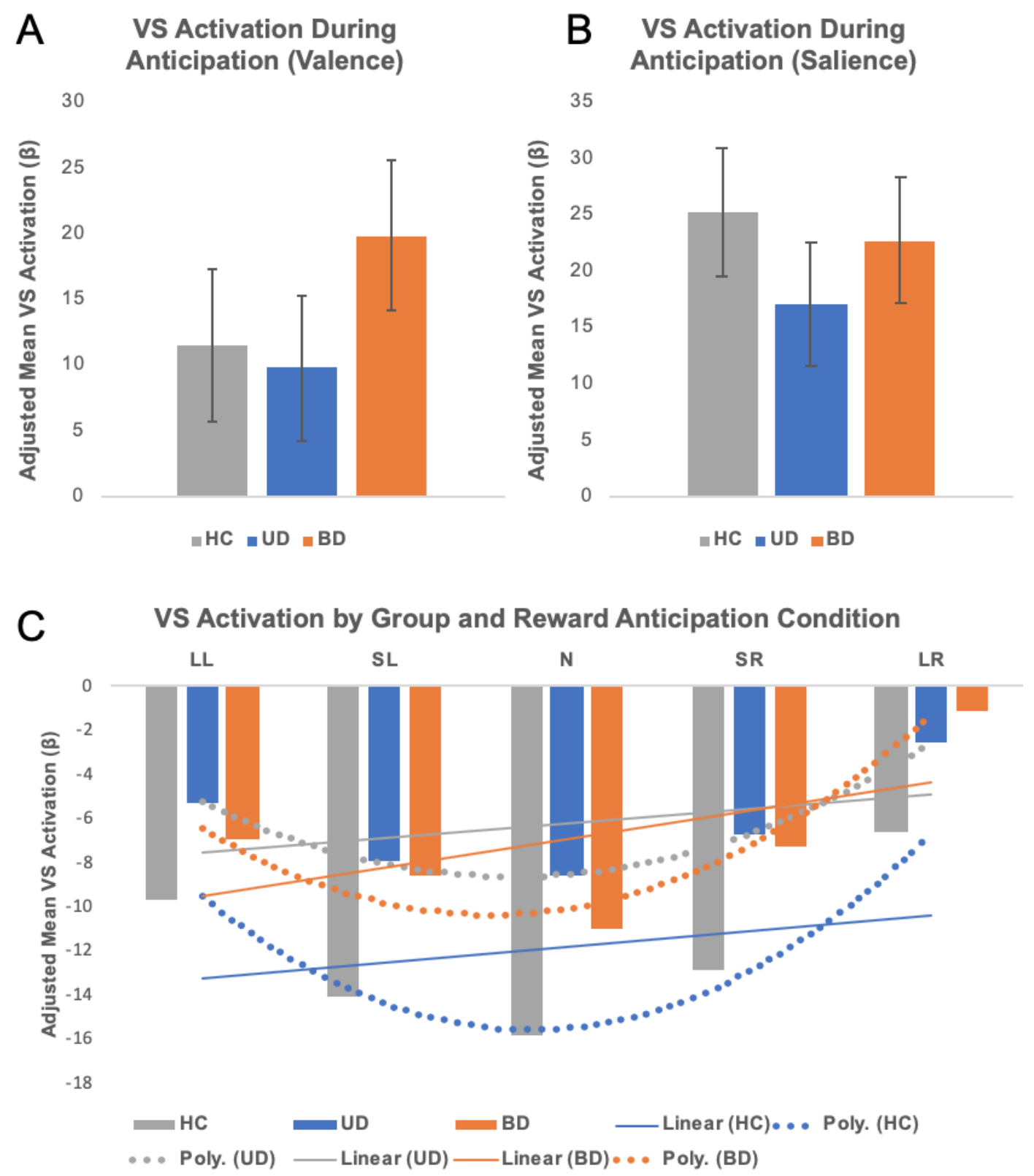

Figure 10. Group differences in VS activation during anticipation. Age, gender, handedness, and hypomanic symptoms were adjusted in the analyses. 
Third, two other ANCOVAs were run to examine the effect of group on OFC and VS activation during feedback, after controlling for age, gender, handedness, and hypomanic symptoms (Figure 11). Levene's tests of homogeneity of variance indicated that there was homogeneity of variances $(p$ 's $>.05)$. Results indicated that there were no statistically significant differences between BD, UD, and HC during feedback in activation in the OFC, $F(2,133)=1.136, p=.324$, partial $\eta^{2}=.017$ (Figure 11A), and the VS, $F(2,133)=0.617, p=.541$, partial $\eta^{2}=.009$ (Figure 11B). Finally, whole-brain analyses did not identify any statistically significant group differences in activation during feedback ( $p$ 's $>.05)$.
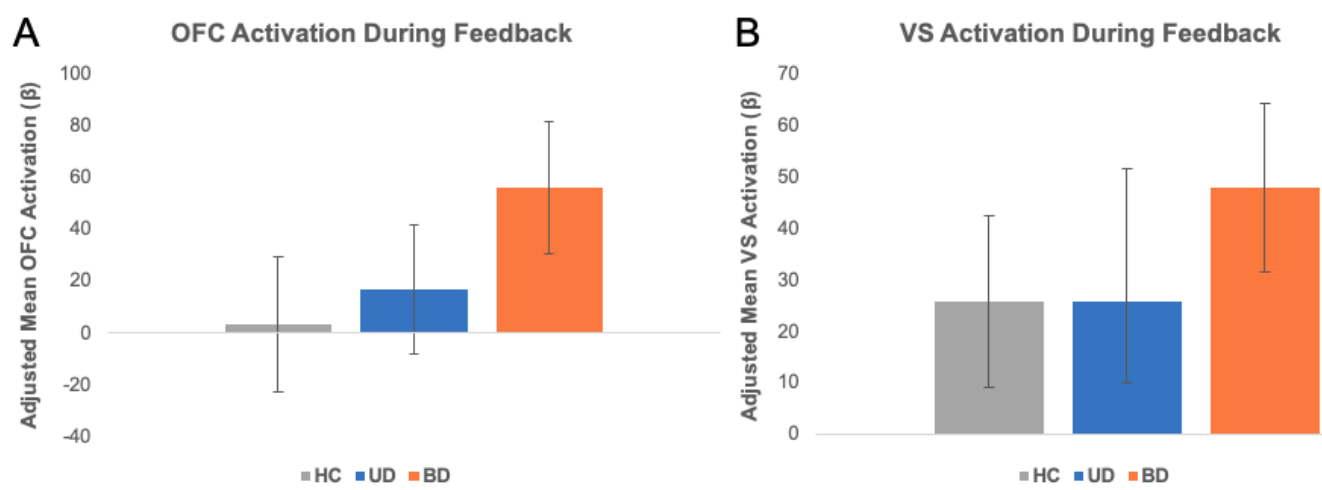

Figure 11. Group differences in OFC and VS activation during feedback. Age, gender, handedness, and hypomanic symptoms were adjusted in the analyses.

\section{Connectivity Analyses}

In addition to activation analyses, connectivity analyses were conducted to examine whether group differences may be reflected in connectivity between the OFC and the VS. An ANCOVA was run to examine the effect of group on OFC-VS functional connectivity, after controlling for age, gender, handedness, and hypomanic symptoms. A Levene's test of homogeneity of variance indicated that there was homogeneity of variances $(p>.05)$. Results suggested that there were no statistically 
significant differences between $\mathrm{BD}, \mathrm{UD}$, and $\mathrm{HC}$ in OFC-VS functional connectivity, $F(2,133)=1.253, p=.289$, partial $\eta^{2}=.018$ (Figure 12).

\section{OFC-VS Functional Connectivity}

0.3
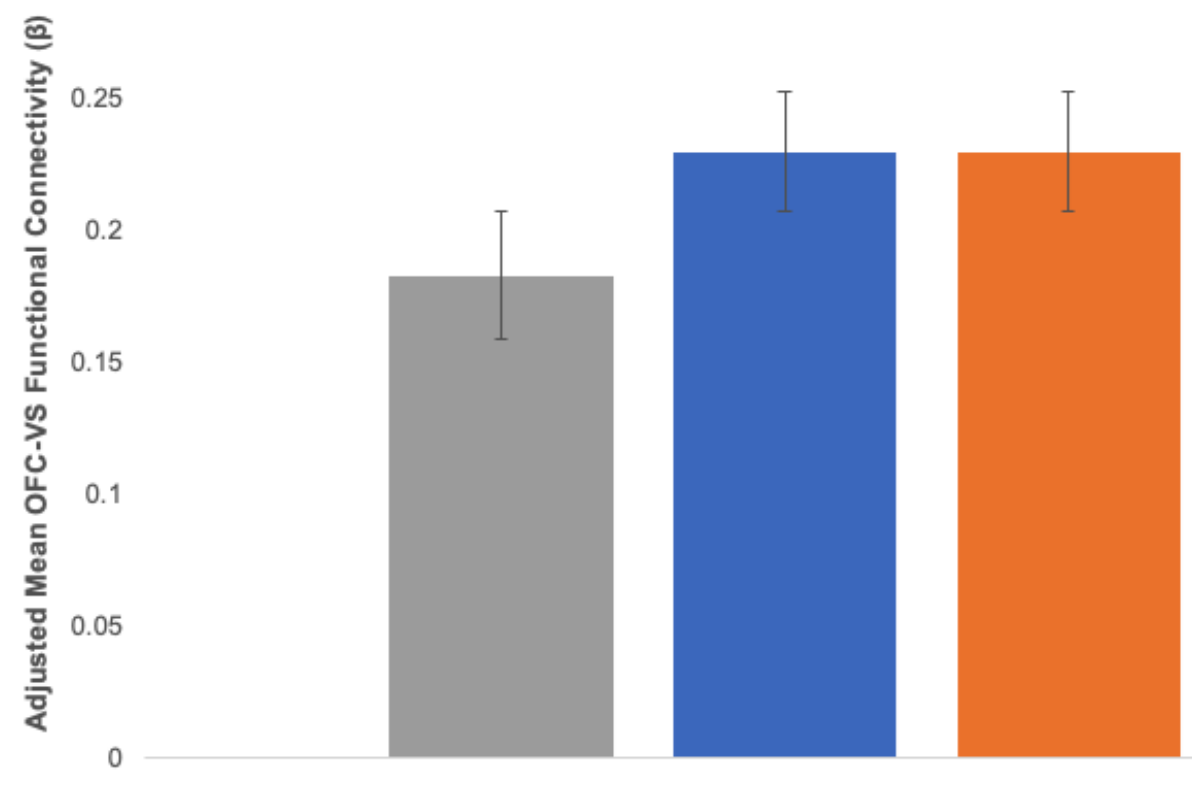

$\because \mathrm{HC}=\mathrm{UD}=\mathrm{BD}$

Figure 12. Group differences in OFC-VS functional connectivity. Age, gender, handedness, and hypomanic symptoms were adjusted in the analyses.

PPI also was used to test whether there were group differences in changes in connectivity strength between the OFC and the VS as a function of reward processing, with OFC as the seed region and VS as the target region. Two ANCOVAs were run to examine the effect of group on OFC-VS connectivity during anticipation (valence and salience; Figure 13), after controlling for age, gender, handedness, and hypomanic symptoms. Levene's tests of homogeneity of variance indicated that there was homogeneity of variances ( $p$ 's > .05). Results indicated that there was a statistically significant difference between $\mathrm{BD}, \mathrm{UD}$, and $\mathrm{HC}$ in OFC-VS connectivity during anticipation (valence), $F(2,133)=3.097, p<.05$, partial $\eta^{2}=.045$ (Figure 13A), but 
not during anticipation (salience), $F(2,133)=0.779, p=.461$, partial $\eta^{2}=.012$ (Figure 13B). Post-hoc tests suggested that there were no statistically significant differences in OFC-VS connectivity during anticipation (valence) between BD and $\mathrm{UD}($ mean difference $=.20, S E=0.09, p=.06), \mathrm{BD}$ and $\mathrm{HC}($ mean difference $=0.17$, $S E=0.09, p=.18$ ), as well as UD and HC (mean difference $=-0.03, S E=0.09, p=$ 1.0), after Bonferroni adjustment.

To further explore what drove the significant group difference in OFC-VS connectivity during anticipation, a two-way mixed ANCOVA was conducted to examine the Group X Condition interaction in predicting OFC-VS connectivity during anticipation (Figure 13C). There was homogeneity of variances (all $p$ 's $>.05$ ), but not homogeneity of covariances (Box's $\mathrm{M}=58.94, p<.01$ ), as assessed by Levene's test of homogeneity of variances and Box's M test, respectively. Mauchly's test of sphericity indicated that the assumption of sphericity was not met for the two-way interaction, $\chi^{2}(9)=21.648, p<.05$. After accounting for age, gender, handedness, and hypomanic symptoms, there was a statistically significant interaction between group and anticipation condition on OFC-VS connectivity, $F(7.385,491.093)=2.321, p<$ .05 , partial $\eta^{2}=.034$ (Figure 13C). Post-hoc tests suggested that there was a statistically significant difference in OFC-VS connectivity during anticipation between BD and HC (mean difference $=-0.089, S E=0.04, p<.05$ ), but not between $\mathrm{BD}$ and UD (mean difference $=-.02, S E=0.03, p=1.00$ ) or UD and HC (mean difference $=-0.07, S E=0.03, p=.13$ ), after Bonferroni adjustment. 
A OFC-Vs Connectivity During

Anticipation (Valence)

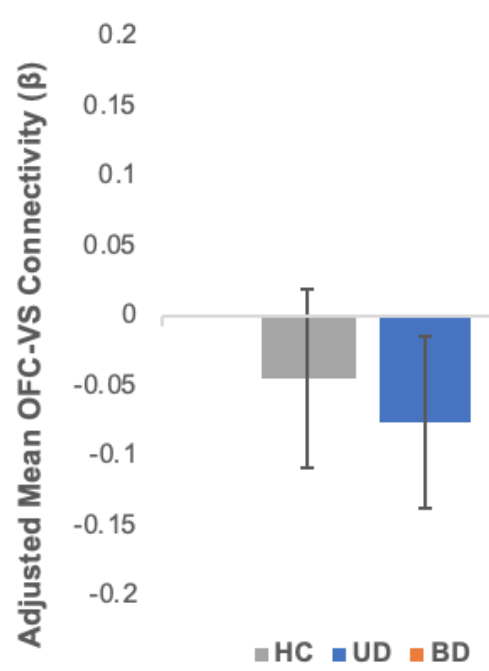

C
B OFC-Vs Connectivity During

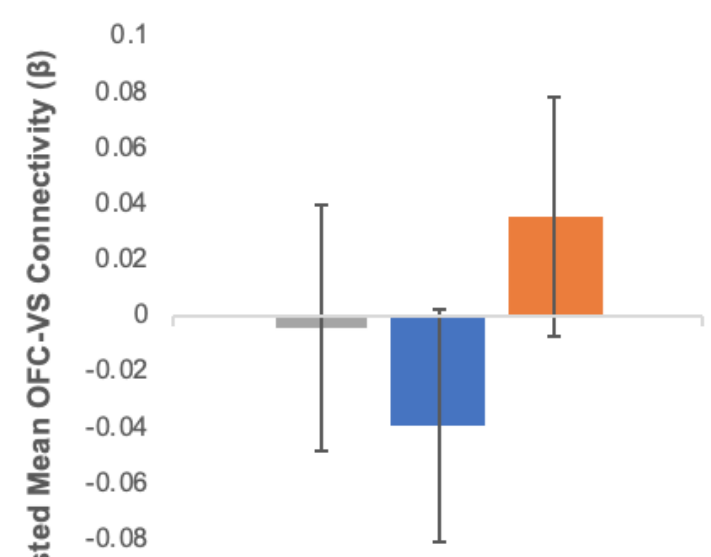

$\because \mathrm{HC} \square \mathrm{UD} \square \mathrm{BD}$

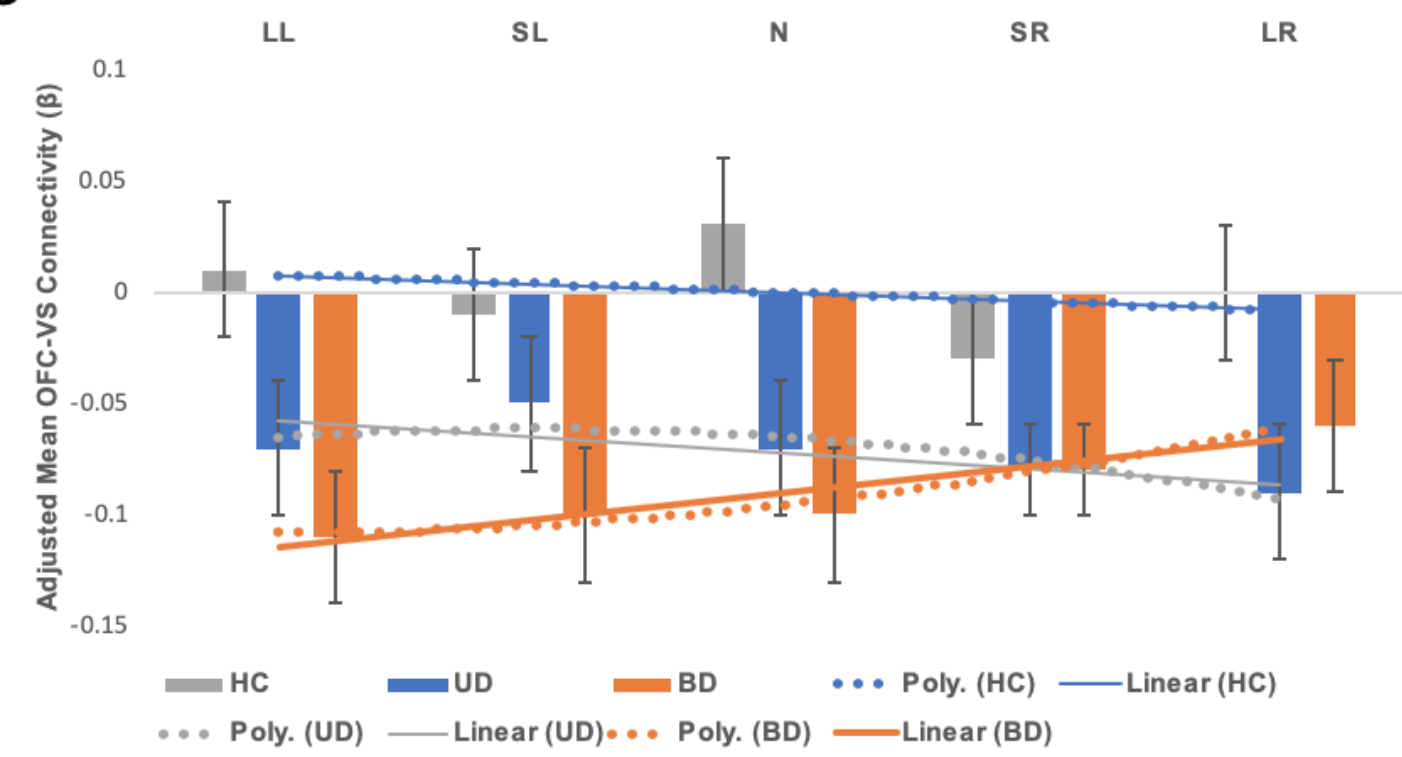

Figure 13. Group differences in OFC-VS connectivity during anticipation. Age, gender, handedness, and hypomanic symptoms were adjusted in the analyses.

Finally, a two-way mixed ANCOVA was conducted to examine the Group X Condition interaction in predicting OFC-VS connectivity during feedback. There was homogeneity of variances and covariances (all $p$ 's > .05), as assessed by Levene's test of homogeneity of variances and Box's M test, respectively. After accounting for age, 
gender, handedness, and hypomanic symptoms, there was no statistically significant interaction between group and feedback condition on OFC-VS connectivity, $F(2,133)$ $=0.295, p=.75$, partial $\eta^{2}=.004$ (Figure 14)

\section{OFC-VS Connectivity by Reward Feedback Condition}

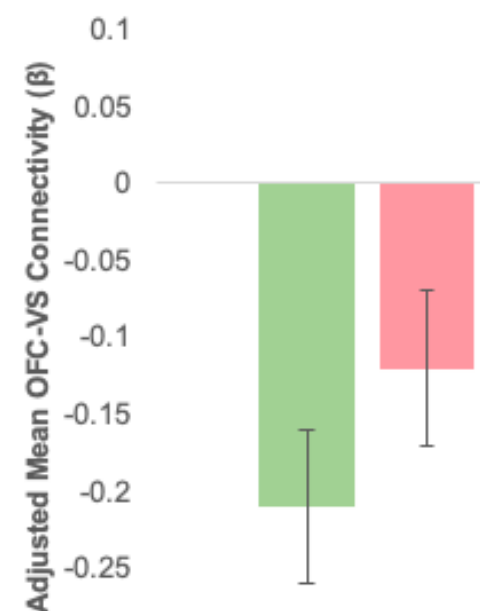

$-0.3$

HC
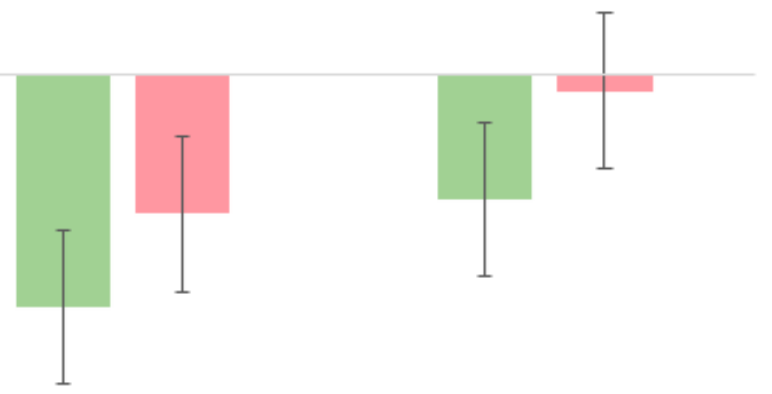

UD

BD

$\varpi$ Hit $=$ Miss

Figure 14. Group differences in OFC-VS connectivity during feedback. Hit = positive feedback; Miss = negative feedback. Age, gender, handedness, and hypomanic symptoms were adjusted in the analyses.

Network Analyses

To provide insight into group differences that are not otherwise found in standard regional approaches, one-way ANCOVAs were conducted to examine differences in spatial correlations between CSNmaps and whole-brain responses during reward processing among BD, UD, and HC. First, I examined whether there were differences in the correlations between CSNmaps and whole-brain responses during anticipation (valence) between $\mathrm{BD}, \mathrm{UD}$, and $\mathrm{HC}$, after controlling for age, gender, handedness, and hypomanic symptoms. Levene's tests of homogeneity of 
variance indicated that there was homogeneity of variances ( $p$ 's > .05). Results indicated that there was a statistically significant difference between BD, UD, and HC in the correlations between whole-brain responses during anticipation (valence) and IC $1, F(2,133)=8.922, p<.01$, partial $\eta^{2}=.118$, IC $2, F(2,133)=7.876, p<.01$, partial $\eta^{2}=.106$, IC $3, F(2,133)=5.615, p<.01$, partial $\eta^{2}=.078$, IC 5, $F(2,133)=$ $3.643, p<.05$, partial $\eta^{2}=.052$, but not IC $7, F(2,133)=0.693, p=.502$, partial $\eta^{2}=$ .010 (Figure 15). Post-hoc tests suggested that HC exhibited stronger correlations between their whole-brain reward anticipation (valence) responses and the CSNmaps than BD (mean difference $=.10, S E=0.02, p<.01)$ and UD (mean difference $=.06$, $S E=0.04, p<.05)$ for IC $1, \mathrm{BD}$ (mean difference $=.11, S E=0.03, p<.01$ ) for IC 2 , as well as BD (mean difference $=.06, S E=0.02, p<.01)$ and UD (mean difference $=$ $.04, S E=0.02, p<.05)$ for IC 3 , after Bonferroni adjustment. Additionally, correlations were significantly lower in $\mathrm{HC}$ than $\mathrm{BD}$ (mean difference $=-.05, S E=$ $0.02, p<.05)$ for IC 5, after Bonferroni adjustment. 


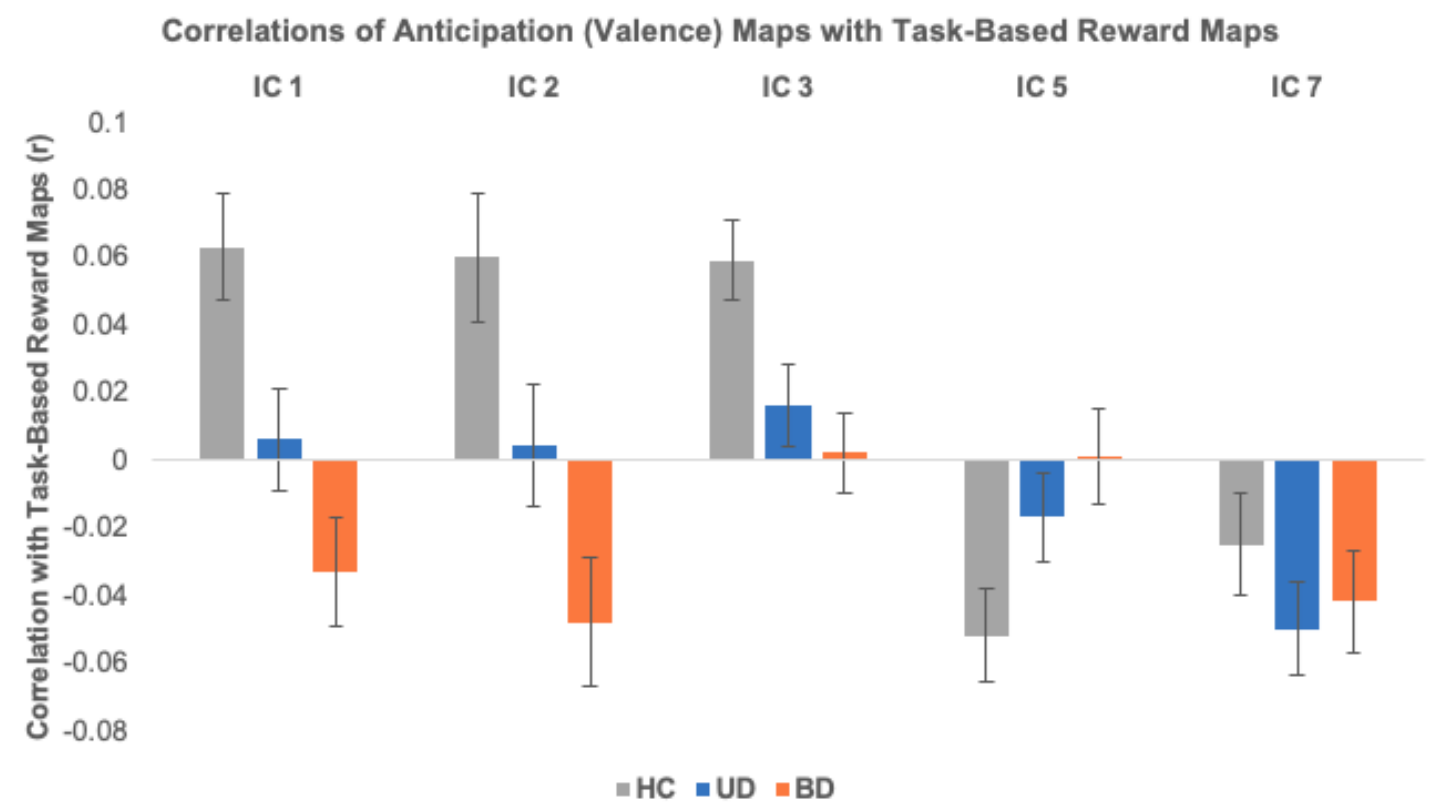

Figure 15. Group differences in spatial correlations between the CSNmaps and the whole-brain responses during anticipation (valence). Age, gender, handedness, and hypomanic symptoms were adjusted in the analyses.

Second, I examined whether there were differences in the correlations between CSNmaps and whole-brain responses during anticipation (salience) between BD, UD, and $\mathrm{HC}$, after controlling for age, gender, handedness, and hypomanic symptoms. Levene's tests of homogeneity of variance indicated that there was homogeneity of variances $(p$ 's $>.05)$. Results indicated that there were no statistically significant differences between $\mathrm{BD}, \mathrm{UD}$, and $\mathrm{HC}$ in the correlations between whole-brain responses during anticipation (salience) and IC $1, F(2,133)=1.645, p=.197$, partial $\eta^{2}=.024$, IC $2, F(2,133)=1.476, p=.232$, partial $\eta^{2}=.022$, IC $3, F(2,133)=$ 0.787, $p=.457$, partial $\eta^{2}=.012$, IC 5, $F(2,133)=0.853, p=.429$, partial $\eta^{2}=.013$, and IC $7, F(2,133)=0.002, p=.998$, partial $\eta^{2}=0.00($ Figure 16$)$. 


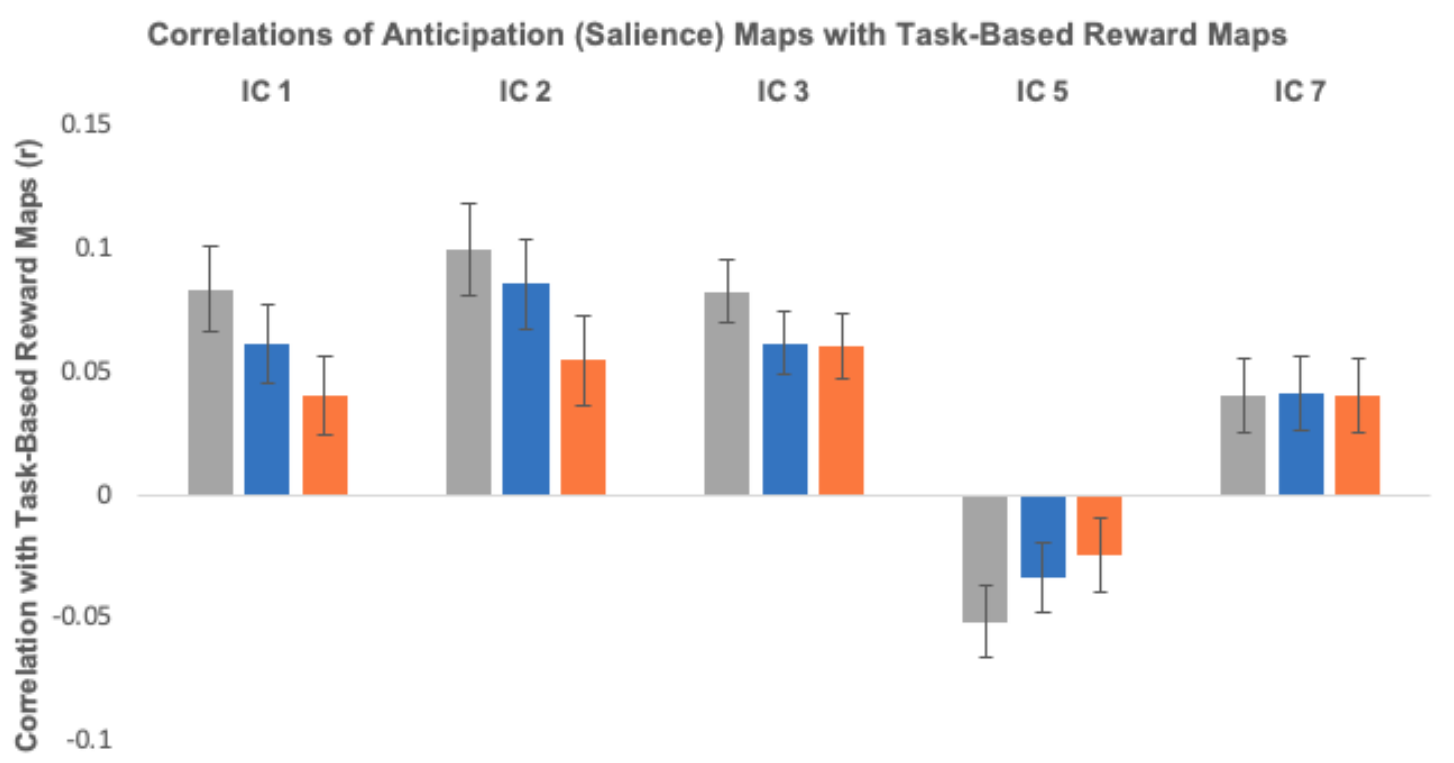

$\varpi \mathrm{HC} \backsim \mathrm{UD}=\mathrm{BD}$

Figure 16. Group differences in spatial correlations between the CSNmaps and the whole-brain responses during anticipation (salience). Age, gender, handedness, and hypomanic symptoms were adjusted in all analyses.

Finally, I examined whether there were differences in the correlations between CSNmaps and whole-brain responses during feedback between BD, UD, and HC, after controlling for age, gender, handedness, and hypomanic symptoms. Levene's tests of homogeneity of variance indicated that there was homogeneity of variances ( $p$ 's $>.05)$. Results indicated that there were no statistically significant differences between $\mathrm{BD}, \mathrm{UD}$, and $\mathrm{HC}$ in the correlations between whole-brain responses during feedback and IC $1, F(2,133)=1.894, p=.154$, partial $\eta^{2}=.028$, IC $2, F(2,133)=$ $1.741, p=.179$, partial $\eta^{2}=.026$, IC $3, F(2,133)=2.318, p=.102$, partial $\eta^{2}=.034$ IC $5, F(2,133)=1.983, p=.142$, partial $\eta^{2}=.029$, and IC $7, F(2,133)=0.722, p=$ .488, partial $\eta^{2}=0.011$ (Figure 17). 


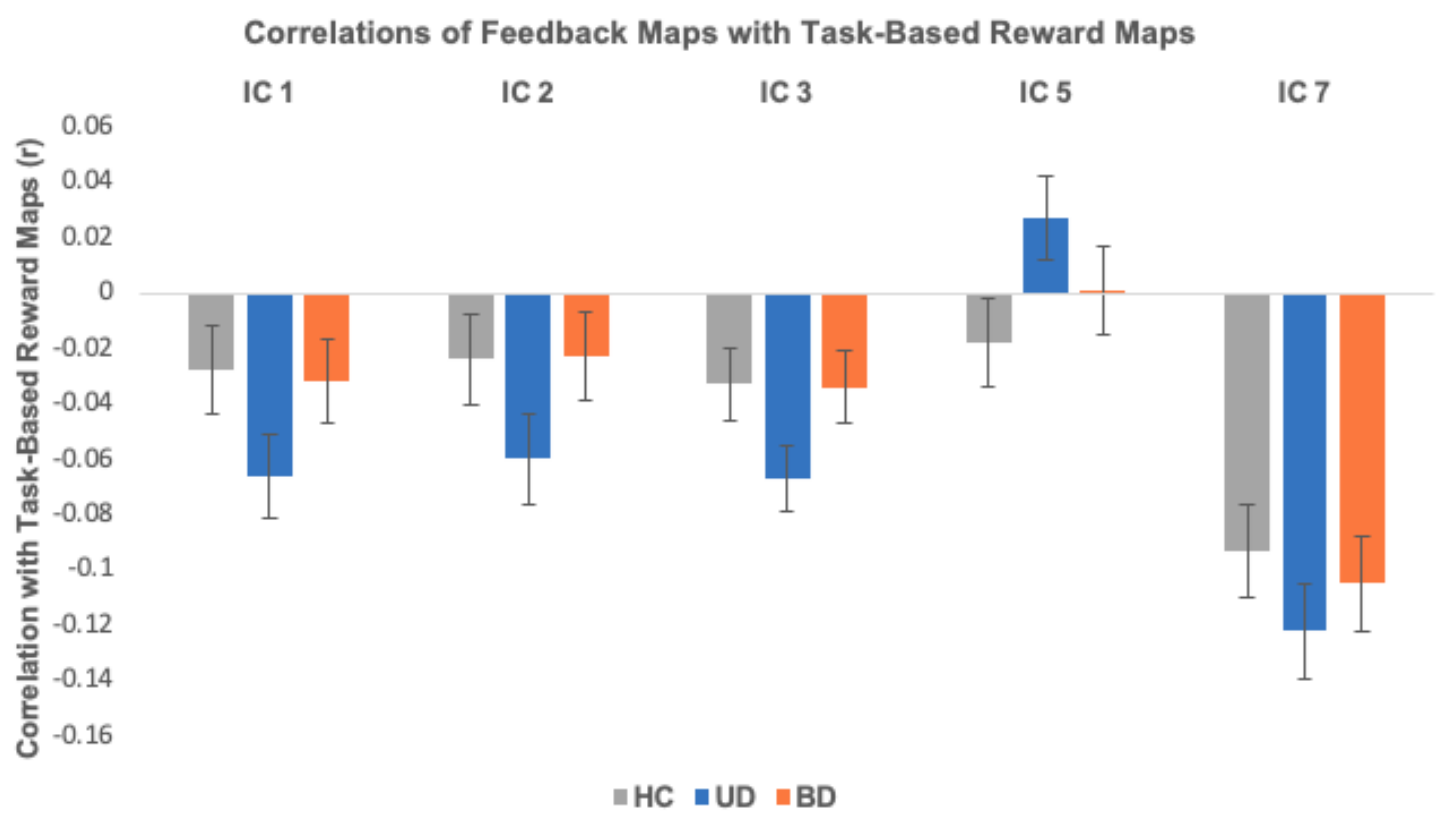

Figure 17. Group differences in spatial correlations between the CSNmaps and the whole-brain responses during feedback. Age, gender, handedness, and hypomanic symptoms were adjusted in the analyses.

\section{Discussion}

The present study examined whether preadolescents with remitted BD or remitted UD exhibit abnormal brain activation or connectivity patterns during reward processing relative to $\mathrm{HC}$ and whether remitted $\mathrm{BD}$ and remitted UD may be distinguished from each other by brain activation and connectivity patterns during reward processing. The results revealed differential connectivity in corticostriatal circuitry during reward processing among $\mathrm{BD}, \mathrm{UD}$, and $\mathrm{HC}$ in preadolescence. Specifically, BD exhibited increases in OFC-VS connectivity during anticipation of larger reward, whereas UD and HC showed no changes in OFC-VS connectivity across anticipation conditions. Furthermore, BD and UD generally showed more abnormal whole-brain responses to anticipation (valence) than $\mathrm{HC}$, as indicated by weaker correlations between a priori established CSNmaps and whole-brain reward 
responses in $\mathrm{BD}$ and $\mathrm{UD}$ relative to $\mathrm{HC}$. Taken together, these findings suggest that preadolescents with $\mathrm{BD}$ and UD exhibit reward processing dysfunction relative to $\mathrm{HC}$ even during remission.

The current findings expand on and clarify earlier work in at least three ways. First, although numerous neuroimaging studies have shown that adults and adolescents with mood disorders exhibit abnormal brain activation patterns during reward processing, no previous study has examined whether such abnormalities begin as early as preadolescence. The current study, the first examination of reward functioning in pre-adolescents with mood disorder using fMRI, provides evidence suggesting that abnormal reward processing also exists in pre-adolescents with mood disorders, despite developmental differences in the reward system between preadolescents, adolescents, and adults (Casey et al., 2008; Chein et al., 2011; Eppinger et al., 2012; Green et al., 1994; Luciana \& Collins, 2012; Somerville et al., 2010; Steinberg, 2007, 2008, 2010; Steinberg et al., 2008, 2009, 2018; Urošević et al., 2012). Second, most neuroimaging studies on reward processing dysfunction in mood disorders have focused on the depressive phase of the disorders. Indeed, only two out of the 41 studies included in Study 1 investigated the remission phase of illness. Studies focusing on the depressive phase, however, cannot address the question of whether reward processing abnormalities are stable characteristics of BD or UD and not simply consequences of depressive mood states, and whether processing dysfunction may be an endophenotype of BD or UD, as one of the criteria for considering a characteristic as an endophenotype is state-independence (Gottesman \& Gould, 2003; Lenzenweger, 2013; G. A. Miller \& Rockstroh, 2013). As the current study only included individuals in remission, the current findings reflect reward processing abnormalities that may manifest outside of acute periods of illness. 
Finally, to my knowledge, this is also the first neuroimaging study that attempts to dissociate valence and salience signals when studying reward processing in mood disorder. The findings suggest that group differences between BD, UD, and $\mathrm{HC}$ are best reflected by valence, rather than salience, as all significant findings in the current study are from valence-based analyses.

Despite the aforementioned evidence for abnormal reward processing in preadolescent mood disorder during remission, I did not find significant differences between preadolescent $\mathrm{BD}, \mathrm{UD}$, and $\mathrm{HC}$ in activation during reward anticipation (valence and salience) and feedback, OFC-VS functional connectivity, OFC-VS connectivity during reward anticipation (salience), or correlations between a priori established corticostriatal activation maps with whole-brain reward-related responses. There are several potential explanations for these null findings. First, given the changes in reward functioning from pre-adolescence to adulthood, findings established in the literature on reward processing dysfunction in adults and adolescents with mood disorders may not be generalizable to pre-adolescents with mood disorders. Therefore, it is possible that certain reward processing abnormalities consistently exhibited by adults and adolescents may not be found in preadolescents. In other words, some reward-related abnormalities may only arise with the developmental changes in brain reward circuitry that occurs in adolescence (Luciana \& Collins, 2012; Urošević et al., 2012). Second, as previous findings mostly relied on adults, who are likely to have longer history of depression than preadolescents, the current null findings could be a function of differences in illness course (e.g., number of recurrences and chronicity). Third, as the current study focused on preadolescence, which is prior to the most common age of onset for BD and UD (Alloy et al., 2016), it is possible that the $\mathrm{HC}$ group included individuals who might ultimately develop BD 
or UD, or that the UD group included individuals who might ultimately convert to $\mathrm{BD}$, obscuring true group differences between BD, UD, and HC. Fourth, as many findings from neuroimaging studies on reward processing in mood disorder are based on participants in acute phases of illness, it is possible that only certain reward processing abnormalities may persist into remission. Finally, given the complexity of reward processing and its neural substrates, regional-based analyses that focus on certain regions of the brain and do not consider the whole brain pattern may obscure important group differences in reward-related brain activity. Therefore, the null results may illustrate a potential weakness with current region-based approaches and highlight the importance of network-based approaches.

In addition to the above theoretical reasons, the null findings also may be attributed to methodological limitations of the current study. First, in the current study, the presence of remitted BD or UD was determined based on information provided by both children and parents, rather than solely children or parents, owing to the notion that different reporters may provide distinct, but equally valid, diagnostic information (Cantwell, Lewinsohn, Rohde, \& Seeley, 1997; Kessler et al., 2001). Nevertheless, it is plausible that brain activation and connectivity patterns associated with reward processing would differ as a function of reporting source. Attempting to understand how to best use and integrate information obtained from multiple informants has challenged researchers of child psychopathology for decades (Cantwell et al., 1997; De Los Reyes \& Kazdin, 2004; Ivens \& Rehm, 1988; Kessler et al., 2001; Klein, 1991; Moretti, Fine, Haley, \& Marriage, 1985; Polanczyk, Salum, Sugaya, Caye, \& Rohde, 2015; Weissman et al., 1987). Parent-child agreement on psychiatric symptoms is often low (Edelbrock, Costello, Dulcan, Conover, \& Kala, 1986; Kashani, Orvaschel, Burk, \& Reid, 1985; Reich, Herjanic, Welner, \& Gandhy, 
1982; Van Roy, Groholt, Heyerdahl, \& Clench-Aas, 2010). To the extent that similar patterns of results emerge across child and parent reports of mood disorder symptoms on the basis of which the BD and UD diagnoses were derived, we can be more confident in the validity of the assessment and the results. Given that no previous research has examined whether brain activation and connectivity patterns associated with reward processing would differ as a function of reporting source, it is important for future studies to explore this question.

Second, although the current study attempted to move towards approaches that focus on connectivity and networks rather than single regions by incorporating CSNmaps into the analyses, other potentially more sensitive and informative analysis methods, such as multi-voxel pattern analysis (MVPA), were not used. Indeed, some studies have demonstrated that MVPA can be applied successfully to neuroimaging data to distinguish adults with BD and UD from HC (Redlich et al., 2014; Zeng et al., 2012). A promising line of future research may examine whether BD, UD, and HC can be distinguished from each other by reward-related brain activation patterns (e.g., striatal responses to reward) using MVPA. Third, although the current study is one of the largest case-control studies on reward processing dysfunction in mood disorder, it is unclear whether the current sample size was large enough to detect small effects. Future research with larger samples will be essential for robustly characterizing the nature of reward processing abnormalities in mood disorders. Finally, case-control designs have inherent limitations, such as their limited explanatory depth and correlational nature (Paulus \& Thompson, 2019; Sedgwick, 2014). Other approaches, such as single-case designs (J. D. Smith, 2012), may be more useful to uncover individual differences among people with mood disorder. 
Taken together, the current study is the first examination of reward processing dysfunction in preadolescents with BD or UD using fMRI and provides novel insight into the nature of reward processing abnormalities associated with mood disorders in preadolescence. Understanding neural circuits underlying BD and UD is crucial for translating foundational knowledge to theoretically-guided interventions designed to target reward processing dysfunction for the prevention or treatment of these debilitating conditions. In theoretical terms, the study addressed crucial knowledge gaps about the reward sensitivity theories of mood disorders by demonstrating that reward processing dysfunction begins as early as pre-adolescence and that reward processing abnormalities may be characteristics of BD and UD that occur even in the remitted state. In applied terms, this study may facilitate current endeavors to identify objective markers of BD and UD by informing understanding of neural circuitry of reward processing implicated in BD and UD. As early onset BD or UD is often associated with remarkably long treatment delays and a persistently pernicious illness course, this study may aid efforts to ensure early accurate diagnosis, which may improve our ability to intervene with appropriate treatments and result in a more benign prognosis and course of illness over the lifespan. 


\section{CHAPTER 4}

\section{GENERAL DISCUSSION}

Given the severity, prevalence, and high rates of recurrence and misdiagnosis of $\mathrm{BD}$ and $\mathrm{UD}$, it is of paramount importance to understand the neurobiological mechanisms underlying these disorders to enhance our ability to diagnose, treat, and prevent them effectively. Many neuroimaging studies have investigated reward processing dysfunction in mood disorders. These studies have led to the common idea that mood disorders are associated with abnormal reward-related responses, particularly in the VS. Yet, the link between mood disorders and reward-related responses in other regions remains inconclusive, thus limiting our understanding of the pathophysiology of mood disorders. To address this issue, I conducted a coordinate-based meta-analysis of neuroimaging studies (fMRI/PET) on reward processing in UD to synthesize the literature in Study 1. The findings argue against the idea that UD is linked to a monolithic deficit within the reward system. Instead, our results demonstrate that UD is associated with opposing abnormalities in the reward circuit: hypo-responses in the VS and hyper-responses in the OFC. These findings help to reconceptualize our understanding of reward processing abnormalities in UD and suggest a role for dysregulated corticostriatal connectivity.

To investigate whether reward-related brain activity can distinguish these two commonly misdiagnosed psychiatric disorders, Study 2 utilized ROI (VS and OFC, as informed by Study 1), whole-brain, connectivity, and network-based analyses of fMRI data to examine the similarities and differences in brain activation patterns between 48 preadolescents with remitted UD, 46 preadolescents with remitted UD, and 48 healthy controls within the context of reward processing, as evoked by the MID task. The results of Study 2 revealed differential connectivity in corticostriatal 
circuitry during reward processing among $\mathrm{BD}, \mathrm{UD}$, and $\mathrm{HC}$ in preadolescence. Specifically, BD exhibited increases in OFC-VS connectivity during anticipation of larger reward, whereas UD and HC showed no changes in OFC-VS connectivity across anticipation conditions. Furthermore, BD and UD generally showed more abnormal whole-brain responses to anticipation (valence) than $\mathrm{HC}$, as indicated by weaker correlations between a priori established CSNmaps and whole-brain reward responses in $\mathrm{BD}$ and $\mathrm{UD}$ relative to $\mathrm{HC}$. Taken together, these findings suggest that preadolescents with $\mathrm{BD}$ and UD exhibit reward processing dysfunction relative to $\mathrm{HC}$ even during remission.

Overall, the results of the dissertation suggest that reward processing dysfunction is a worthy focus of future research on mood disorders. Given that no previous research has examined whether brain activation and connectivity patterns associated with reward processing would differ as a function of reporting source, it is important for future studies to explore this question. In addition, future research may consider examining whether BD, UD, and $\mathrm{HC}$ can be distinguished from each other by reward-related brain activation patterns (e.g., striatal responses to reward) using MVPA. Moreover, future studies with larger samples and different mood states will be essential for robustly characterizing the nature of reward processing abnormalities in mood disorders. Furthermore, longitudinal studies will be necessary to understand how reward processing abnormalities may predict recurrences, recovery, and conversion to more severe forms of illness (e.g., from UD to BD). Finally, other approaches, such as single-case designs (J. D. Smith, 2012), may be useful to uncover individual differences among people with mood disorder.

While improving diagnostic accuracy and treatment for individuals diagnosed with mood disorders is critically important, it is equally important to develop effective 
early identification and prevention strategies for individuals at risk for developing mood disorders. Consequently, future studies may employ genetic high-risk designs and fMRI to examine the similarities and differences in reward-related brain activation patterns between healthy offspring of parents with BD, UD, or no psychopathology. Importantly, the findings from such a study and Study 2 might synergistically inform our understanding of neurobiological markers associated with risk (i.e., when abnormalities are found in high-risk and affected individuals but not normal controls), resilience (when abnormalities are found only in high-risk individuals but not affected individuals and normal controls), or illness (when abnormalities are found only in affected individuals but not high-risk individuals and normal controls). By focusing on individuals who have not yet exhibited, but are at high genetic risk for BD or UD, rather than those who have already developed them, we may be able to identify neurobiological markers of risk, rather than expression, of these disorders. As a result, it may aid efforts to ensure early identification of risks for the disorders, which may improve our ability to intervene with targeted intervention, and thus, prevent disorder expression or result in a more benign prognosis over the lifespan. 


\section{REFERENCES}

Achenbach, T. M. (2009). The Achenbach system of empirically based assessment (ASEBA): Development, findings, theory, and applications. University of Vermont, Research Center for Children, Youth, \& Families.

Admon, R., \& Pizzagalli, D. A. (2015a). Corticostriatal pathways contribute to the natural time course of positive mood. Nature Communications, 6, 10065. https://doi.org/10.1038/ncomms10065

Admon, R., \& Pizzagalli, D. A. (2015b). Dysfunctional reward processing in depression. Current Opinion in Psychology, 4, 114-118. https://doi.org/10.1016/j.copsyc.2014.12.011

Alloy, L. B., Nusslock, R., \& Boland, E. M. (2015). The development and course of bipolar spectrum disorders: An integrated reward and circadian rhythm dysregulation model. Annual Review of Clinical Psychology, 11, 213-250. https://doi.org/10.1146/annurev-clinpsy-032814-112902

Alloy, L. B., Olino, T., Freed, R. D., \& Nusslock, R. (2016). Role of reward sensitivity and processing in major depressive and bipolar spectrum disorders. Behavior Therapy, 47(5), 600-621. https://doi.org/10.1016/j.beth.2016.02.014

Almeida, J. R. C. de, Versace, A., Mechelli, A., Hassel, S., Quevedo, K., Kupfer, D. J., \& Phillips, M. L. (2009). Abnormal amygdala-prefrontal effective connectivity to happy faces differentiates bipolar from major depression. Biological Psychiatry, 66(5), 451-459. https://doi.org/10.1016/j.biopsych.2009.03.024

Altshuler, L. L., Post, R. M., Leverich, G. S., Mikalauskas, K., Rosoff, A., \& Ackerman, L. (1995). Antidepressant-induced mania and cycle acceleration: A controversy revisited. The American Journal of Psychiatry, 152(8), 11301138.

Andersson, J. L., Hutton, C., Ashburner, J., Turner, R., \& Friston, K. (2001). Modeling geometric deformations in EPI time series. NeuroImage, 13(5), 903-919. https://doi.org/10.1006/nimg.2001.0746

Andreasen, N. C., Rice, J., Endicott, J., Reich, T., \& Coryell, W. (1986). The family history approach to diagnosis. How useful is it? Archives of General Psychiatry, 43(5), 421-429.

Angst, J., Azorin, J.-M., Bowden, C. L., Perugi, G., Vieta, E., Gamma, A., ... Group, for the B. S. (2011). Prevalence and characteristics of undiagnosed bipolar disorders in patients with a major depressive episode: The BRIDGE study. Archives of General Psychiatry, 68(8), 791-799. https://doi.org/10.1001/archgenpsychiatry.2011.87

Arrondo, G., Segarra, N., Metastasio, A., Ziauddeen, H., Spencer, J., Reinders, N. R., ... Murray, G. K. (2015). Reduction in ventral striatal activity when anticipating a reward in depression and schizophrenia: A replicated crossdiagnostic finding. Frontiers in Psychology, 6. https://doi.org/10.3389/fpsyg.2015.01280

Avants, B., Epstein, C., Grossman, M., \& Gee, J. (2008). Symmetric diffeomorphic image registration with cross-correlation: Evaluating automated labeling of elderly and neurodegenerative brain. Medical Image Analysis, 12(1), 26-41. https://doi.org/10.1016/j.media.2007.06.004

Barch, D. M., Albaugh, M. D., Avenevoli, S., Chang, L., Clark, D. B., Glantz, M. D., ... Sher, K. J. (2017). Demographic, physical and mental health assessments in the adolescent brain and cognitive development study: 
Rationale and description. Developmental Cognitive Neuroscience. https://doi.org/10.1016/j.den.2017.10.010

Bartra, O., McGuire, J. T., \& Kable, J. W. (2013). The valuation system: A coordinate-based meta-analysis of BOLD fMRI experiments examining neural correlates of subjective value. NeuroImage, 76, 412-427. https://doi.org/10.1016/j.neuroimage.2013.02.063

Beckmann, C. F., \& Smith, S. M. (2005). Tensorial extensions of independent component analysis for multisubject FMRI analysis. NeuroImage, 25(1), 294 311. https://doi.org/10.1016/j.neuroimage.2004.10.043

Beljan, P., Webb, J. T., Amend, E. R., Web, N. E., Goerss, J., \& Olenchak, F. R. (2006). Misdiagnosis and dual diagnoses of gifted children and adults: ADHD, bipolar, OCD, Asperger's, depression, and other disorders. Gifted and Talented International, 21(2), 83-86. https://doi.org/10.1080/15332276.2006.11673478

Berridge, K. C., \& Kringelbach, M. L. (2015). Pleasure systems in the brain. Neuron, 86(3), 646-664. https://doi.org/10.1016/j.neuron.2015.02.018

Bhargava Raman, R. P., Sheshadri, S. P., Janardhan Reddy, Y. C., Girimaji, S. C., Srinath, S., \& Raghunandan, V. N. G. P. (2007). Is bipolar II disorder misdiagnosed as major depressive disorder in children? Journal of Affective Disorders, 98(3), 263-266. https://doi.org/10.1016/j.jad.2006.08.006

Bild, D. E., Bluemke, D. A., Burke, G. L., Detrano, R., Diez Roux, A. V., Folsom, A. R., ... Tracy, R. P. (2002). Multi-ethnic study of atherosclerosis: Objectives and design. American Journal of Epidemiology, 156(9), 871-881. https://doi.org/10.1093/aje/kwf113

Bjork, J. M., Straub, L. K., Provost, R. G., \& Neale, M. C. (2017). The ABCD study of neurodevelopment: Identifying neurocircuit targets for prevention and treatment of adolescent substance abuse. Current Treatment Options in Psychiatry, 4(2), 196-209. https://doi.org/10.1007/s40501-017-0108-y

Bowden, C. L. (2005). A different depression: Clinical distinctions between bipolar and unipolar depression. Journal of Affective Disorders, 84(2-3), 117-125. https://doi.org/10.1016/S0165-0327(03)00194-0

Bremner, J. D., Vythilingam, M., Vermetten, E., \& Charney, D. S. (2007). Effects of antidepressant treatment on neural correlates of emotional and neutral declarative verbal memory in depression. Journal of Affective Disorders, 101(1-3), 99-111. https://doi.org/10.1016/j.jad.2006.10.028

Bress, J. N., Foti, D., Kotov, R., Klein, D. N., \& Hajcak, G. (2013). Blunted neural response to rewards prospectively predicts depression in adolescent girls. Psychophysiology, 50(1), 74-81. https://doi.org/10.1111/j.14698986.2012.01485.x

Bromet, E., Andrade, L. H., Hwang, I., Sampson, N. A., Alonso, J., de Girolamo, G., ... Kessler, R. C. (2011). Cross-national epidemiology of DSM-IV major depressive episode. BMC Medicine, 9, 90. https://doi.org/10.1186/1741-70159-90

Bürger, C., Redlich, R., Grotegerd, D., Meinert, S., Dohm, K., Schneider, I., ... Dannlowski, U. (2017). Differential abnormal pattern of anterior cingulate gyrus activation in unipolar and bipolar depression: An fMRI and pattern classification approach. Neuropsychopharmacology, 42(7), 1399-1408. https://doi.org/10.1038/npp.2017.36

Button, K. S., Ioannidis, J. P. A., Mokrysz, C., Nosek, B. A., Flint, J., Robinson, E. S. J., \& Munafò, M. R. (2013). Power failure: Why small sample size 
undermines the reliability of neuroscience. Nature Reviews Neuroscience, 14(5), 365-376. https://doi.org/10.1038/nrn3475

Cantwell, D. P., Lewinsohn, P. M., Rohde, P., \& Seeley, J. R. (1997). Correspondence between adolescent report and parent report of psychiatric diagnostic data. Journal of the American Academy of Child and Adolescent Psychiatry, 36(5), 610-619. https://doi.org/10.1097/00004583-199705000-00011

Carlson, G. A. (2000). The challenge of diagnosing depression in childhood and adolescence. Journal of Affective Disorders, 61 Suppl 1, 3-8.

Carver, C. S., \& White, T. L. (1994). Behavioral inhibition, behavioral activation, and affective responses to impending reward and punishment: The BIS/BAS Scales. Journal of Personality and Social Psychology, 67(2), 319-333. https://doi.org/10.1037/0022-3514.67.2.319

Casey, B. J., Cannonier, T., Conley, M. I., Cohen, A. O., Barch, D. M., Heitzeg, M. M., ... Dale, A. M. (2018). The Adolescent Brain Cognitive Development (ABCD) study: Imaging acquisition across 21 sites. Developmental Cognitive Neuroscience. https://doi.org/10.1016/j.dcn.2018.03.001

Casey, B. J., Jones, R. M., \& Hare, T. A. (2008). The adolescent brain. Annals of the New York Academy of Sciences, 1124, 111-126. https://doi.org/10.1196/annals.1440.010

Chang, K. (2009). Challenges in the diagnosis and treatment of pediatric bipolar depression. Dialogues in Clinical Neuroscience, 11(1), 73-80.

Chantiluke, K., Halari, R., Simic, M., Pariante, C. M., Papadopoulos, A., Giampietro, V., \& Rubia, K. (2012). Fronto-striato-cerebellar dysregulation in adolescents with depression during motivated attention. Biological Psychiatry, 71(1), 5967. https://doi.org/10.1016/j.biopsych.2011.09.005

Chase, H. W., Kumar, P., Eickhoff, S. B., \& Dombrovski, A. Y. (2015). Reinforcement learning models and their neural correlates: An activation likelihood estimation meta-analysis. Cognitive, Affective, \& Behavioral Neuroscience, 15(2), 435-459. https://doi.org/10.3758/s13415-015-0338-7

Chase, H. W., Nusslock, R., Almeida, J. R. C., Forbes, E. E., LaBarbara, E. J., \& Phillips, M. L. (2013). Dissociable patterns of abnormal frontal cortical activation during anticipation of an uncertain reward or loss in bipolar versus major depression. Bipolar Disorders, 15(8), 839-854. https://doi.org/10.1111/bdi.12132

Chatham, C. H., Frank, M. J., \& Badre, D. (2014). Corticostriatal output gating during selection from working memory. Neuron, 81(4), 930-942. https://doi.org/10.1016/j.neuron.2014.01.002

Chein, J., Albert, D., O’Brien, L., Uckert, K., \& Steinberg, L. (2011). Peers increase adolescent risk taking by enhancing activity in the brain's reward circuitry. Developmental Science, 14(2), F1-F10. https://doi.org/10.1111/j.14677687.2010.01035.x

Chilakamarri, J. K., Filkowski, M. M., \& Ghaemi, S. N. (2011). Misdiagnosis of bipolar disorder in children and adolescents: A comparison with ADHD and major depressive disorder. Annals of Clinical Psychiatry: Official Journal of the American Academy of Clinical Psychiatrists, 23(1), 25-29.

Chumbley, J. R., \& Friston, K. J. (2009). False discovery rate revisited: FDR and topological inference using Gaussian random fields. NeuroImage, 44(1), 6270. https://doi.org/10.1016/j.neuroimage.2008.05.021

Clark, D. B., Fisher, C. B., Bookheimer, S., Brown, S. A., Evans, J. H., Hopfer, C., ... Yurgelun-Todd, D. (2017). Biomedical ethics and clinical oversight in 
multisite observational neuroimaging studies with children and adolescents: The ABCD experience. Developmental Cognitive Neuroscience. https://doi.org/10.1016/j.den.2017.06.005

Cooper, J. C., \& Knutson, B. (2008). Valence and salience contribute to nucleus accumbens activation. NeuroImage, 39(1), 538-547. https://doi.org/10.1016/j.neuroimage.2007.08.009

De Los Reyes, A., \& Kazdin, A. E. (2004). Measuring informant discrepancies in clinical child research. Psychological Assessment, 16(3), 330-334. https://doi.org/10.1037/1040-3590.16.3.330

Delgado, M. R., Beer, J. S., Fellows, L. K., Huettel, S. A., Platt, M. L., Quirk, G. J., \& Schiller, D. (2016). Viewpoints: Dialogues on the functional role of the ventromedial prefrontal cortex. Nature Neuroscience, 19(12), 1545-1552. https://doi.org/10.1038/nn.4438

Delgado, M. R., Nystrom, L. E., Fissell, C., Noll, D. C., \& Fiez, J. A. (2000). Tracking the hemodynamic responses to reward and punishment in the striatum. Journal of Neurophysiology, 84(6), 3072-3077. https://doi.org/10.1152/jn.2000.84.6.3072

Demenescu, L. R., Renken, R., Kortekaas, R., van Tol, M.-J., Marsman, J. B. C., van Buchem, M. A., ... Aleman, A. (2011). Neural correlates of perception of emotional facial expressions in out-patients with mild-to-moderate depression and anxiety. A multicenter fMRI study. Psychological Medicine, 41(11), 2253-2264. https://doi.org/10.1017/S0033291711000596

Der-Avakian, A., \& Markou, A. (2012). The neurobiology of anhedonia and other reward-related deficits. Trends in Neurosciences, 35(1), 68-77. https://doi.org/10.1016/j.tins.2011.11.005

Desikan, R. S., Ségonne, F., Fischl, B., Quinn, B. T., Dickerson, B. C., Blacker, D., ... Killiany, R. J. (2006). An automated labeling system for subdividing the human cerebral cortex on MRI scans into gyral based regions of interest. NeuroImage, 31(3), 968-980. https://doi.org/10.1016/j.neuroimage.2006.01.021

Dichter, G. S., Felder, J. N., Petty, C., Bizzell, J., Ernst, M., \& Smoski, M. J. (2009). The effects of psychotherapy on neural responses to rewards in major depression. Biological Psychiatry, 66(9), 886-897. https://doi.org/10.1016/j.biopsych.2009.06.021

Dichter, G. S., Kozink, R. V., McClernon, F. J., \& Smoski, M. J. (2012). Remitted major depression is characterized by reward network hyperactivation during reward anticipation and hypoactivation during reward outcomes. Journal of Affective Disorders, 136(3), 1126-1134. https://doi.org/10.1016/j.jad.2011.09.048

Diehl, M. M., Lempert, K. M., Parr, A. C., Ballard, I., Steele, V. R., \& Smith, D. V. (2018). Toward an integrative perspective on the neural mechanisms underlying persistent maladaptive behaviors. European Journal of Neuroscience, 48(3), 1870-1883. https://doi.org/10.1111/ejn.14083

Drysdale, A. T., Grosenick, L., Downar, J., Dunlop, K., Mansouri, F., Meng, Y., ... Liston, C. (2017). Resting-state connectivity biomarkers define neurophysiological subtypes of depression. Nature Medicine, 23(1), 28-38. https://doi.org/10.1038/nm.4246

Edelbrock, C., Costello, A. J., Dulcan, M. K., Conover, N. C., \& Kala, R. (1986). Parent-child agreement on child psychiatric symptoms assessed via structured 
interview. Journal of Child Psychology and Psychiatry, and Allied Disciplines, 27(2), 181-190.

Eickhoff, S. B., Bzdok, D., Laird, A. R., Kurth, F., \& Fox, P. T. (2012). Activation Likelihood Estimation meta-analysis revisited. Neuroimage, 59(3), 23492361. https://doi.org/10.1016/j.neuroimage.2011.09.017

Eickhoff, S. B., Laird, A. R., Fox, P. M., Lancaster, J. L., \& Fox, P. T. (2017). Implementation errors in the GingerALE software: Description and recommendations. Human Brain Mapping, 38(1), 7-11. https://doi.org/10.1002/hbm.23342

Eickhoff, S. B., Laird, A. R., Grefkes, C., Wang, L. E., Zilles, K., \& Fox, P. T. (2009). Coordinate-based activation likelihood estimation meta-analysis of neuroimaging data: A random-effects approach based on empirical estimates of spatial uncertainty. Human Brain Mapping, 30(9), 2907-2926. https://doi.org/10.1002/hbm.20718

Eickhoff, S. B., Nichols, T. E., Laird, A. R., Hoffstaedter, F., Amunts, K., Fox, P. T., ... Eickhoff, C. R. (2016). Behavior, sensitivity, and power of activation likelihood estimation characterized by massive empirical simulation. NeuroImage, 137, 70-85. https://doi.org/10.1016/j.neuroimage.2016.04.072

Eklund, A., Nichols, T. E., \& Knutsson, H. (2016). Cluster failure: Why fMRI inferences for spatial extent have inflated false-positive rates. Proceedings of the National Academy of Sciences, 113(28), 7900-7905. https://doi.org/10.1073/pnas.1602413113

Elliott, R., Rubinsztein, J. S., Sahakian, B. J., \& Dolan, R. J. (2002). The neural basis of mood-congruent processing biases in depression. Archives of General Psychiatry, 59(7), 597-604. https://doi.org/10.1001/archpsyc.59.7.597

Engelmann, J. B., Berns, G. S., \& Dunlop, B. W. (2017). Hyper-responsivity to losses in the anterior insula during economic choice scales with depression severity. Psychological Medicine, 47(16), 2879-2891. https://doi.org/10.1017/S0033291717001428

Eppinger, B., Nystrom, L. E., \& Cohen, J. D. (2012). Reduced sensitivity to immediate reward during decision-making in older than younger adults. PLOS ONE, 7(5), e36953. https://doi.org/10.1371/journal.pone.0036953

Eshel, N., \& Roiser, J. P. (2010). Reward and punishment processing in depression. Biological Psychiatry, 68(2), 118-124. https://doi.org/10.1016/j.biopsych.2010.01.027

Esteban, O., Markiewicz, C., Blair, R. W., Moodie, C., Isik, A. I., Aliaga, A. E., ... Gorgolewski, K. J. (2018). FMRIPrep: A robust preprocessing pipeline for functional MRI. BioRxiv, 306951. https://doi.org/10.1101/306951

Felger, J. C., Li, Z., Haroon, E., Woolwine, B. J., Jung, M. Y., Hu, X., \& Miller, A. H. (2016). Inflammation is associated with decreased functional connectivity within corticostriatal reward circuitry in depression. Molecular Psychiatry, 21(10), 1358-1365. https://doi.org/10.1038/mp.2015.168

Ferenczi, E. A., Zalocusky, K. A., Liston, C., Grosenick, L., Warden, M. R., Amatya, D., ... Deisseroth, K. (2016). Prefrontal cortical regulation of brainwide circuit dynamics and reward-related behavior. Science, 351(6268), aac9698. https://doi.org/10.1126/science.aac9698

Fonov, V., Evans, A., McKinstry, R., Almli, C., \& Collins, D. (2009). Unbiased nonlinear average age-appropriate brain templates from birth to adulthood. NeuroImage, 47, S102. https://doi.org/10.1016/S1053-8119(09)70884-5 
Forbes, E. E., Hariri, A. R., Martin, S. L., Silk, J. S., Moyles, D. L., Fisher, P. M., ... Dahl, R. E. (2009). Altered striatal activation predicting real-world positive affect in adolescent major depressive disorder. The American Journal of Psychiatry, 166(1), 64-73. https://doi.org/10.1176/appi.ajp.2008.07081336

Forbes, E. E., May, J. C., Siegle, G. J., Ladouceur, C. D., Ryan, N. D., Carter, C. S., ... Dahl, R. E. (2006). Reward-related decision-making in pediatric major depressive disorder: An fMRI study. Journal of Child Psychology and Psychiatry and Allied Disciplines, 47(10), 1031-1040. https://doi.org/10.1111/j.1469-7610.2006.01673.x

Fournier, J. C., Keener, M. T., Mullin, B. C., Hafeman, D. M., LaBarbara, E. J., Stiffler, R. S., ... Phillips, M. L. (2013). Heterogeneity of amygdala response in major depressive disorder: The impact of lifetime sub-threshold mania. Psychological Medicine, 43(2), 293-302. https://doi.org/10.1017/S0033291712000918

Friston, K. J., Buechel, C., Fink, G. R., Morris, J., Rolls, E., \& Dolan, R. J. (1997). Psychophysiological and modulatory interactions in neuroimaging. NeuroImage, 6(3), 218-229. https://doi.org/10.1006/nimg.1997.0291

Friston, K. J., Harrison, L., \& Penny, W. (2003). Dynamic causal modelling. NeuroImage, 19(4), 1273-1302. https://doi.org/10.1016/S10538119(03)00202-7

Fu, C. H. Y., Williams, S. C. R., Brammer, M. J., Suckling, J., Kim, J., Cleare, A. J., ... Bullmore, E. T. (2007). Neural responses to happy facial expressions in major depression following antidepressant treatment. The American Journal of Psychiatry, 164(4), 599-607. https://doi.org/10.1176/ajp.2007.164.4.599

Fu, C. H. Y., Williams, S. C. R., Cleare, A. J., Brammer, M. J., Walsh, N. D., Kim, J., ... Bullmore, E. T. (2004). Attenuation of the neural response to sad faces in major depression by antidepressant treatment: a prospective, event-related functional magnetic resonance imaging study. Archives of General Psychiatry, 61(9), 877-889. https://doi.org/10.1001/archpsyc.61.9.877

Fu, C. H. Y., Williams, S. C. R., Cleare, A. J., Scott, J., Mitterschiffthaler, M. T., Walsh, N. D., ... Murray, R. M. (2008). Neural responses to sad facial expressions in major depression following cognitive behavioral therapy. Biological Psychiatry, 64(6), 505-512. https://doi.org/10.1016/j.biopsych.2008.04.033

Garavan, H., Bartsch, H., Conway, K., Decastro, A., Goldstein, R., Heeringa, S., ... Zahs, D. (2018). Recruiting the ABCD sample: Design considerations and procedures. Developmental Cognitive Neuroscience. https://doi.org/10.1016/j.den.2018.04.004

Geller, B., \& Luby, J. (1997). Child and adolescent bipolar disorder: A review of the past 10 years. Journal of the American Academy of Child \& Adolescent Psychiatry, 36(9), 1168-1176. https://doi.org/10.1097/00004583-19970900000008

Ghaemi, S. N., Boiman, E. E., \& Goodwin, F. K. (2000). Diagnosing bipolar disorder and the effect of antidepressants: A naturalistic study. The Journal of Clinical Psychiatry, 61(10), 804-808; quiz 809.

Ghaemi, S. N., Sachs, G. S., Chiou, A. M., Pandurangi, A. K., \& Goodwin, F. K. (1999). Is bipolar disorder still underdiagnosed? Are antidepressants overutilized? Journal of Affective Disorders, 52(1), 135-144. https://doi.org/10.1016/S0165-0327(98)00076-7 
Gold, J. M., Waltz, J. A., Matveeva, T. M., Kasanova, Z., Strauss, G. P., Herbener, E. S., ... Frank, M. J. (2012). Negative symptoms and the failure to represent the expected reward value of actions: Behavioral and computational modeling evidence. Archives of General Psychiatry, 69(2), 129-138. https://doi.org/10.1001/archgenpsychiatry.2011.1269

Goodwin, F. K., \& Jamison, K. R. (2007). Manic-depressive illness: Bipolar disorders and recurrent depression. New York: Oxford University Press.

Gorgolewski, K. J., Auer, T., Calhoun, V. D., Craddock, R. C., Das, S., Duff, E. P., ... Poldrack, R. A. (2016). The brain imaging data structure, a format for organizing and describing outputs of neuroimaging experiments. Scientific Data, 3, 160044. https://doi.org/10.1038/sdata.2016.44

Gotlib, I. H., Hamilton, J. P., Cooney, R. E., Singh, M. K., Henry, M. L., \& Joormann, J. (2010). Neural processing of reward and loss in girls at risk for major depression. Archives of General Psychiatry, 67(4), 380-387. https://doi.org/10.1001/archgenpsychiatry.2010.13

Gotlib, I. H., Sivers, H., Gabrieli, J. D. E., Whitfield-Gabrieli, S., Goldin, P., Minor, K. L., \& Canli, T. (2005). Subgenual anterior cingulate activation to valenced emotional stimuli in major depression. Neuroreport, 16(16), 1731-1734.

Gottesman, I. I., \& Gould, T. D. (2003). The endophenotype concept in psychiatry: Eymology and strategic intentions. The American Journal of Psychiatry, 160(4), 636-645. https://doi.org/10.1176/appi.ajp.160.4.636

Gradin, V. B., Pérez, A., MacFarlane, J. A., Cavin, I., Waiter, G., Engelmann, J., ... Steele, J. D. (2015). Abnormal brain responses to social fairness in depression: An fMRI study using the Ultimatum Game. Psychological Medicine, 45(6), 1241-1251. https://doi.org/10.1017/S0033291714002347

Green, L., Fry, A. F., \& Myerson, J. (1994). Discounting of delayed rewards: A lifespan comparison. Psychological Science, 5(1), 33-36. https://doi.org/10.1111/j.1467-9280.1994.tb00610.x

Greve, D. N., \& Fischl, B. (2009). Accurate and robust brain image alignment using boundary-based registration. NeuroImage, 48(1), 63-72. https://doi.org/10.1016/j.neuroimage.2009.06.060

Groenewold, N. A., Opmeer, E. M., de Jonge, P., Aleman, A., \& Costafreda, S. G. (2013). Emotional valence modulates brain functional abnormalities in depression: Evidence from a meta-analysis of fMRI studies. Neuroscience \& Biobehavioral Reviews, 37(2), 152-163. https://doi.org/10.1016/j.neubiorev.2012.11.015

Haber, S. N. (2016). Corticostriatal circuitry. Dialogues in Clinical Neuroscience, 18(1), 7-21.

Haber, S. N., \& Knutson, B. (2010). The reward circuit: Linking primate anatomy and human imaging. Neuropsychopharmacology, 35(1), 4-26. https://doi.org/10.1038/npp.2009.129

Hall, G. B. C., Milne, A. M. B., \& MacQueen, G. M. (2014). An fMRI study of reward circuitry in patients with minimal or extensive history of major depression. European Archives of Psychiatry and Clinical Neuroscience, 264(3), 187-198. https://doi.org/10.1007/s00406-013-0437-9

Hamilton, J. P., Etkin, A., Furman, D. J., Lemus, M. G., Johnson, R. F., \& Gotlib, I. H. (2012). Functional neuroimaging of major depressive disorder: A metaanalysis and new integration of base line activation and neural response data. The American Journal of Psychiatry, 169(7), 693-703. https://doi.org/10.1176/appi.ajp.2012.11071105 
Hanson, J. L., Hariri, A. R., \& Williamson, D. E. (2015). Blunted ventral striatum development in adolescence reflects emotional neglect and predicts depressive symptoms. Biological Psychiatry, 78(9), 598-605.

https://doi.org/10.1016/j.biopsych.2015.05.010

Hanson, J. L., Knodt, A. R., Brigidi, B. D., \& Hariri, A. R. (2017). Heightened connectivity between the ventral striatum and medial prefrontal cortex as a biomarker for stress-related psychopathology: Understanding interactive effects of early and more recent stress. Psychological Medicine, 1-9. https://doi.org/10.1017/S0033291717003348

Hare, T. A., O’Doherty, J., Camerer, C. F., Schultz, W., \& Rangel, A. (2008). Dissociating the role of the orbitofrontal cortex and the striatum in the computation of goal values and prediction errors. Journal of Neuroscience, 28(22), 5623-5630. https://doi.org/10.1523/JNEUROSCI.1309-08.2008

Hayden, B. Y., Parikh, P. C., Deaner, R. O., \& Platt, M. L. (2007). Economic principles motivating social attention in humans. Proceedings of the Royal Society B: Biological Sciences, 274(1619), 1751-1756. https://doi.org/10.1098/rspb.2007.0368

Heshmati, M., \& Russo, S. J. (2015). Anhedonia and the brain reward circuitry in depression. Current Behavioral Neuroscience Reports, 2(3), 146-153. https://doi.org/10.1007/s40473-015-0044-3

Hirschfeld, R. M. A., Lewis, L., \& Vornik, L. A. (2003). Perceptions and impact of bipolar disorder: How far have we really come? Results of the national depressive and manic-depressive association 2000 survey of individuals with bipolar disorder. The Journal of Clinical Psychiatry, 64(2), 161-174.

Holm, S. M., Forbes, E. E., Ryan, N. D., Phillips, M. L., Tarr, J. A., \& Dahl, R. E. (2009). Reward-related brain function and sleep in pre/early pubertal and mid/late pubertal adolescents. Journal of Adolescent Health, 45(4), 326-334. https://doi.org/10.1016/j.jadohealth.2009.04.001

Ivens, C., \& Rehm, L. P. (1988). Assessment of childhood depression: Correspondence between reports by child, mother, and father. Journal of the American Academy of Child and Adolescent Psychiatry, 27(6), 738-747. https://doi.org/10.1097/00004583-198811000-00012

Jenkinson, M., Bannister, P., Brady, M., \& Smith, S. (2002). Improved optimization for the robust and accurate linear registration and motion correction of brain images. NeuroImage, 17(2), 825-841. https://doi.org/10.1006/nimg.2002.1132

Jia, X. Z., Zhao, N., Barton, B., Burciu, R., Carriere, N., Cerasa, A., ... Zang, Y. F. (2018). Small effect size leads to reproducibility failure in resting-state fMRI studies. BioRxiv. https://doi.org/10.1101/285171

Johnston, B. A., Tolomeo, S., Gradin, V., Christmas, D., Matthews, K., \& Steele, J. D. (2015). Failure of hippocampal deactivation during loss events in treatment-resistant depression. Brain: A Journal of Neurology, 138(Pt 9), 2766-2776. https://doi.org/10.1093/brain/awv177

Kahnt, T., Park, S. Q., Haynes, J.-D., \& Tobler, P. N. (2014). Disentangling neural representations of value and salience in the human brain. Proceedings of the National Academy of Sciences of the United States of America, 111(13), 50005005. https://doi.org/10.1073/pnas.1320189111

Kaiser, R. H., Andrews-Hanna, J. R., Wager, T. D., \& Pizzagalli, D. A. (2015). Largescale network dysfunction in major depressive disorder: A meta-analysis of resting-state functional connectivity. JAMA Psychiatry, 72(6), 603-611. https://doi.org/10.1001/jamapsychiatry.2015.0071 
Kashani, J. H., Orvaschel, H., Burk, J. P., \& Reid, J. C. (1985). Informant variance: The issue of parent-child disagreement. Journal of the American Academy of Child Psychiatry, 24(4), 437-441.

Keedwell, P. A., Andrew, C., Williams, S. C. R., Brammer, M. J., \& Phillips, M. L. (2005). A double dissociation of ventromedial prefrontal cortical responses to sad and happy stimuli in depressed and healthy individuals. Biological Psychiatry, 58(6), 495-503. https://doi.org/10.1016/j.biopsych.2005.04.035

Keren, H., O’Callaghan, G., Vidal-Ribas, P., Buzzell, G. A., Brotman, M. A., Leibenluft, E., ... Stringaris, A. (2018). Reward processing in depression: A conceptual and meta-analytic review across fMRI and EEG studies. American Journal of Psychiatry, 175(11), 1111-1120. https://doi.org/10.1176/appi.ajp.2018.17101124

Kessler, R. C., Avenevoli, S., \& Ries Merikangas, K. (2001). Mood disorders in children and adolescents: An epidemiologic perspective. Biological Psychiatry, 49(12), 1002-1014. https://doi.org/10.1016/S00063223(01)01129-5

Klein, R. G. (1991). Parent-child agreement in clinical assessment of anxiety and other psychopathology: A review. Journal of Anxiety Disorders, 5(2), 187198. https://doi.org/10.1016/0887-6185(91)90028-R

Knutson, B., Bhanji, J. P., Cooney, R. E., Atlas, L. Y., \& Gotlib, I. H. (2008). Neural responses to monetary incentives in major depression. Biological Psychiatry, 63(7), 686-692. https://doi.org/10.1016/j.biopsych.2007.07.023

Knutson, B., Westdorp, A., Kaiser, E., \& Hommer, D. (2000). FMRI visualization of brain activity during a monetary incentive delay task. NeuroImage, 12(1), 2027. https://doi.org/10.1006/nimg.2000.0593

Kringelbach, M. L. (2005). The human orbitofrontal cortex: Linking reward to hedonic experience. Nature Reviews Neuroscience, 6(9), 691-702. https://doi.org/10.1038/nrn1747

Kringelbach, M. L., \& Rolls, E. T. (2004). The functional neuroanatomy of the human orbitofrontal cortex: Evidence from neuroimaging and neuropsychology.

Progress in Neurobiology, 72(5), 341-372.

https://doi.org/10.1016/j.pneurobio.2004.03.006

Kumari, V., Mitterschiffthaler, M. T., Teasdale, J. D., Malhi, G. S., Brown, R. G., Giampietro, V., ... Sharma, T. (2003). Neural abnormalities during cognitive generation of affect in treatment-resistant depression. Biological Psychiatry, 54(8), 777-791.

Lancaster, J. L., Tordesillas-Gutiérrez, D., Martinez, M., Salinas, F., Evans, A., Zilles, K., ... Fox, P. T. (2007). Bias between MNI and Talairach coordinates analyzed using the ICBM-152 brain template. Human Brain Mapping, 28(11), 1194-1205. https://doi.org/10.1002/hbm.20345

Laurent, H. K., \& Ablow, J. C. (2013). A face a mother could love: Depressionrelated maternal neural responses to infant emotion faces. Social Neuroscience, 8(3), 228-239. https://doi.org/10.1080/17470919.2012.762039

Lenzenweger, M. F. (2013). Endophenotype, intermediate phenotype, biomarker: Definitions, concept comparisons, clarifications. Depression and Anxiety, 30(3), 185-189. https://doi.org/10.1002/da.22042

Leonpacher, A. K., Liebers, D., Pirooznia, M., Jancic, D., MacKinnon, D. F., Mondimore, F. M., ... Goes, F. S. (2015). Distinguishing bipolar from unipolar depression: The importance of clinical symptoms and illness features. 
Psychological Medicine, 45(11), 2437-2446.

https://doi.org/10.1017/S0033291715000446

Levy, D. J., \& Glimcher, P. W. (2012). The root of all value: A neural common currency for choice. Current Opinion in Neurobiology, 22(6), 1027-1038. https://doi.org/10.1016/j.conb.2012.06.001

Lewis, P. A., Critchley, H. D., Rotshtein, P., \& Dolan, R. J. (2007). Neural correlates of processing valence and arousal in affective words. Cerebral Cortex, 17(3), 742-748. https://doi.org/10.1093/cercor/bhk024

Linke, J., King, A. V., Rietschel, M., Strohmaier, J., Hennerici, M., Gass, A., ... Wessa, M. (2012). Increased medial orbitofrontal and amygdala activation: Evidence for a systems-level endophenotype of bipolar I disorder. The American Journal of Psychiatry, 169(3), 316-325. https://doi.org/10.1176/appi.ajp.2011.11050711

Lish, J. D., Dime-Meenan, S., Whybrow, P. C., Price, R. A., \& Hirschfeld, R. M. (1994). The National Depressive and Manic-depressive Association (DMDA) survey of bipolar members. Journal of Affective Disorders, 31(4), 281-294.

Litt, A., Plassmann, H., Shiv, B., \& Rangel, A. (2011). Dissociating valuation and saliency signals during decision-making. Cerebral Cortex, 21(1), 95-102. https://doi.org/10.1093/cercor/bhq065

Liu, W.-H., Valton, V., Wang, L.-Z., Zhu, Y.-H., \& Roiser, J. P. (2017). Association between habenula dysfunction and motivational symptoms in unmedicated major depressive disorder. Social Cognitive and Affective Neuroscience, 12(9), 1520-1533. https://doi.org/10.1093/scan/nsx074

Luby, J. L. (2013). Treatment of anxiety and depression in the preschool period. Journal of the American Academy of Child \& Adolescent Psychiatry, 52(4), 346-358. https://doi.org/10.1016/j.jaac.2013.01.011

Luciana, M., \& Collins, P. F. (2012). Incentive motivation, cognitive control, and the adolescent brain: Is it time for a paradigm shift? Child Development Perspectives, 6(4), 392-399. https://doi.org/10.1111/j.17508606.2012.00252.x

Maia, T. V., \& Frank, M. J. (2011). From reinforcement learning models to psychiatric and neurological disorders. Nature Neuroscience, 14(2), 154-162. https://doi.org/10.1038/nn.2723

Mattson, W. I., Hyde, L. W., Shaw, D. S., Forbes, E. E., \& Monk, C. S. (2016). Clinical neuroprediction: Amygdala reactivity predicts depressive symptoms 2 years later. Social Cognitive and Affective Neuroscience, 11(6), 892-898. https://doi.org/10.1093/scan/nsw018

Mazziotta, J. C., Toga, A. W., Evans, A., Fox, P., \& Lancaster, J. (1995). A probabilistic atlas of the human brain: Theory and rationale for its development. The International Consortium for Brain Mapping (ICBM). NeuroImage, 2(2, Part A), 89-101. https://doi.org/10.1006/nimg.1995.1012

McLaren, D. G., Ries, M. L., Xu, G., \& Johnson, S. C. (2012). A generalized form of context-dependent psychophysiological interactions (gPPI): A comparison to standard approaches. NeuroImage, 61(4), 1277-1286. https://doi.org/10.1016/j.neuroimage.2012.03.068

Merikangas, K. R., Jin, R., He, J.-P., Kessler, R. C., Lee, S., Sampson, N. A., ... Zarkov, Z. (2011). Prevalence and correlates of bipolar spectrum disorder in the world mental health survey initiative. Archives of General Psychiatry, 68(3), 241-251. https://doi.org/10.1001/archgenpsychiatry.2011.12 
Miller, C. H., Hamilton, J. P., Sacchet, M. D., \& Gotlib, I. H. (2015). Meta-analysis of functional neuroimaging of major depressive disorder in youth. JAMA Psychiatry, 72(10), 1045-1053. https://doi.org/10.1001/jamapsychiatry.2015.1376

Miller, G. A., \& Rockstroh, B. (2013). Endophenotypes in psychopathology research: Where do we stand? Annual Review of Clinical Psychology, 9, 177-213. https://doi.org/10.1146/annurev-clinpsy-050212-185540

Mitterschiffthaler, M. T., Kumari, V., Malhi, G. S., Brown, R. G., Giampietro, V. P., Brammer, M. J., ... Sharma, T. (2003). Neural response to pleasant stimuli in anhedonia: An fMRI study. Neuroreport, 14(2), 177-182. https://doi.org/10.1097/01.wnr.0000053758.76853.cc

Moher, D., Liberati, A., Tetzlaff, J., Altman, D. G., \& PRISMA Group. (2009). Preferred reporting items for systematic reviews and meta-analyses: The PRISMA statement. Annals of Internal Medicine, 151, 264-269.

Moretti, M. M., Fine, S., Haley, G., \& Marriage, K. (1985). Childhood and adolescent depression: Child-report versus parent-report information. Journal of the American Academy of Child Psychiatry, 24(3), 298-302.

Morgan, J. K., Olino, T. M., McMakin, D. L., Ryan, N. D., \& Forbes, E. E. (2013). Neural response to reward as a predictor of increases in depressive symptoms in adolescence. Neurobiology of Disease, 52, 66-74. https://doi.org/10.1016/j.nbd.2012.03.039

Müller, V. I., Cieslik, E. C., Laird, A. R., Fox, P. T., Radua, J., Mataix-Cols, D., ... Eickhoff, S. B. (2017). Ten simple rules for neuroimaging meta-analysis. Neuroscience and Biobehavioral Reviews. https://doi.org/10.1016/j.neubiorev.2017.11.012

Müller, V. I., Cieslik, E. C., Serbanescu, I., Laird, A. R., Fox, P. T., \& Eickhoff, S. B. (2017). Altered brain activity in unipolar depression revisited: Meta-analyses of neuroimaging studies. JAMA Psychiatry, 74(1), 47-55. https://doi.org/10.1001/jamapsychiatry.2016.2783

Murrough, J. W., Collins, K. A., Fields, J., DeWilde, K. E., Phillips, M. L., Mathew, S. J., ... Iosifescu, D. V. (2015). Regulation of neural responses to emotion perception by ketamine in individuals with treatment-resistant major depressive disorder. Translational Psychiatry, 5(2), e509. https://doi.org/10.1038/tp.2015.10

Murty, V. P., Shermohammed, M., Smith, D. V., Carter, R. M., Huettel, S. A., \& Adcock, R. A. (2014). Resting state networks distinguish human ventral tegmental area from substantia nigra. NeuroImage, 100, 580-589. https://doi.org/10.1016/j.neuroimage.2014.06.047

Naesström, M., Blomstedt, P., \& Bodlund, O. (2016). A systematic review of psychiatric indications for deep brain stimulation, with focus on major depressive and obsessive-compulsive disorder. Nordic Journal of Psychiatry, 70(7), 483-491. https://doi.org/10.3109/08039488.2016.1162846

Naranjo, C. A., Tremblay, L. K., \& Busto, U. E. (2001). The role of the brain reward system in depression. Progress in Neuro-Psychopharmacology and Biological Psychiatry, 25(4), 781-823. https://doi.org/10.1016/S0278-5846(01)00156-7

Ng, T. H., Alloy, L. B., \& Smith, D. V. (2018). Meta-analysis of reward processing in major depressive disorder: Distinct abnormalities within the reward circuit? BioRxiv, 332981. https://doi.org/10.1101/332981

Nichols, T. E., Das, S., Eickhoff, S. B., Evans, A. C., Glatard, T., Hanke, M., ... Yeo, B. T. T. (2017). Best practices in data analysis and sharing in neuroimaging 
using MRI. Nature Neuroscience, 20(3), 299-303.

https://doi.org/10.1038/nn.4500

Nielson, D. M., Pereira, F., Zheng, C. Y., Migineishvili, N., Lee, J. A., Thomas, A. G., \& Bandettini, P. A. (2018). Detecting and harmonizing scanner differences in the ABCD study - Annual release 1.0. BioRxiv, 309260. https://doi.org/10.1101/309260

Nusslock, R., \& Alloy, L. B. (2017). Reward processing and mood-related symptoms: An $\mathrm{RDoC}$ and translational neuroscience perspective. Journal of Affective Disorders, 216, 3-16. https://doi.org/10.1016/j.jad.2017.02.001

Nusslock, R., Almeida, J. R., Forbes, E. E., Versace, A., Frank, E., Labarbara, E. J., ... Phillips, M. L. (2012). Waiting to win: Elevated striatal and orbitofrontal cortical activity during reward anticipation in euthymic bipolar disorder adults. Bipolar Disorders, 14(3), 249-260. https://doi.org/10.1111/j.13995618.2012.01012.x

Ojemann, J. G., Akbudak, E., Snyder, A. Z., McKinstry, R. C., Raichle, M. E., \& Conturo, T. E. (1997). Anatomic localization and quantitative analysis of gradient refocused echo-planar fMRI susceptibility artifacts. NeuroImage, 6(3), 156-167. https://doi.org/10.1006/nimg.1997.0289

Oldfield, R. C. (1971). The assessment and analysis of handedness: The Edinburgh inventory. Neuropsychologia, 9(1), 97-113. https://doi.org/10.1016/00283932(71)90067-4

Olino, T. M., McMakin, D. L., Morgan, J. K., Silk, J. S., Birmaher, B., Axelson, D. A., ... Forbes, E. E. (2014). Reduced reward anticipation in youth at high-risk for unipolar depression: A preliminary study. Developmental Cognitive Neuroscience, 8, 55-64. https://doi.org/10.1016/j.dcn.2013.11.005

Padoa-Schioppa, C. (2011). Neurobiology of economic choice: A good-based model. Annual Review of Neuroscience, 34, 333-359. https://doi.org/10.1146/annurev-neuro-061010-113648

Padoa-Schioppa, C., \& Conen, K. E. (2017). Orbitofrontal cortex: A neural circuit for economic decisions. Neuron, 96(4), 736-754. https://doi.org/10.1016/j.neuron.2017.09.031

Pagliaccio, D., Luking, K. R., Anokhin, A. P., Gotlib, I. H., Hayden, E. P., Olino, T. M., ... Barch, D. M. (2016). Revising the BIS/BAS to study development: Measurement invariance and normative effects of age and sex from childhood through adulthood. Psychological Assessment, 28(4), 429-442. https://doi.org/10.1037/pas0000186

Palmer, S. M., Crewther, S. G., Carey, L. M., \& START Project Team. (2014). A meta-analysis of changes in brain activity in clinical depression. Frontiers in Human Neuroscience, 8, 1045. https://doi.org/10.3389/fnhum.2014.01045

Paulus, M. P., \& Thompson, W. K. (2019). The challenges and opportunities of small effects: The new normal in academic psychiatry. JAMA Psychiatry. https://doi.org/10.1001/jamapsychiatry.2018.4540

Pendergast, L. L., Youngstrom, E. A., Merkitch, K. G., Moore, K. A., Black, C., Abramson, L., \& Alloy, L. B. (2014). Differentiating bipolar disorder from unipolar depression and ADHD: The utility of the General Behavior Inventory. Psychological Assessment, 26(1), 195-206. https://doi.org/10.1037/a0035138

Perlis, R. H., Ostacher, M. J., Goldberg, J. F., Miklowitz, D. J., Friedman, E., Calabrese, J., ... Sachs, G. S. (2010). Transition to mania during treatment of 
bipolar depression. Neuropsychopharmacology, 35(13), 2545-2552. https://doi.org/10.1038/npp.2010.122

Peters, J., \& Büchel, C. (2010). Neural representations of subjective reward value. Behavioural Brain Research, 213(2), 135-141. https://doi.org/10.1016/j.bbr.2010.04.031

Phelps, E. A., Lempert, K. M., \& Sokol-Hessner, P. (2014). Emotion and decision making: Multiple modulatory neural circuits. Annual Review of Neuroscience, 37, 263-287. https://doi.org/10.1146/annurev-neuro-071013-014119

Phillips, M. L., Drevets, W. C., Rauch, S. L., \& Lane, R. (2003). Neurobiology of emotion perception II: Implications for major psychiatric disorders. Biological Psychiatry, 54(5), 515-528.

Phillips, M. L., \& Swartz, H. A. (2014). A critical appraisal of neuroimaging studies of bipolar disorder: Toward a new conceptualization of underlying neural circuitry and roadmap for future research. American Journal of Psychiatry, 171(8), 829-843. https://doi.org/10.1176/appi.ajp.2014.13081008

Pickens, C. L., Saddoris, M. P., Setlow, B., Gallagher, M., Holland, P. C., \& Schoenbaum, G. (2003). Different roles for orbitofrontal cortex and basolateral amygdala in a reinforcer devaluation task. Journal of Neuroscience, 23(35), 11078-11084. https://doi.org/10.1523/JNEUROSCI.2335-11078.2003

Pizzagalli, D. A., Holmes, A. J., Dillon, D. G., Goetz, E. L., Birk, J. L., Bogdan, R., ... Fava, M. (2009). Reduced caudate and nucleus accumbens response to rewards in unmedicated subjects with major depressive disorder. The American Journal of Psychiatry, 166(6), 702-710. https://doi.org/10.1176/appi.ajp.2008.08081201

Polanczyk, G. V., Salum, G. A., Sugaya, L. S., Caye, A., \& Rohde, L. A. (2015). Annual Research Review: A meta-analysis of the worldwide prevalence of mental disorders in children and adolescents. Journal of Child Psychology and Psychiatry, 56(3), 345-365. https://doi.org/10.1111/jcpp.12381

Poldrack, R. A., Baker, C. I., Durnez, J., Gorgolewski, K. J., Matthews, P. M., Munafò, M. R., ... Yarkoni, T. (2017). Scanning the horizon: Towards transparent and reproducible neuroimaging research. Nature Reviews Neuroscience, 18(2), 115-126. https://doi.org/10.1038/nrn.2016.167

Post, R. M., Leverich, G. S., Kupka, R. W., Keck, J. P., McElroy, S. L., Altshuler, L. L., ... Nolen, W. A. (2010). Early-onset bipolar disorder and treatment delay are risk factors for poor outcome in adulthood. The Journal of Clinical Psychiatry, 71(7), 864-872. https://doi.org/10.4088/JCP.08m04994yel

Power, J. D., Barnes, K. A., Snyder, A. Z., Schlaggar, B. L., \& Petersen, S. E. (2012). Spurious but systematic correlations in functional connectivity MRI networks arise from subject motion. Neuroimage, 59(3), 2142-2154. https://doi.org/10.1016/j.neuroimage.2011.10.018

Price, J. L., \& Drevets, W. C. (2010). Neurocircuitry of mood disorders. Neuropsychopharmacology, 35(1), 192-216. https://doi.org/10.1038/npp.2009.104

Pruim, R. H. R., Mennes, M., van Rooij, D., Llera, A., Buitelaar, J. K., \& Beckmann, C. F. (2015). ICA-AROMA: A robust ICA-based strategy for removing motion artifacts from fMRI data. NeuroImage, 112, 267-277. https://doi.org/10.1016/j.neuroimage.2015.02.064 
Rangel, A., Camerer, C., \& Montague, P. R. (2008). A framework for studying the neurobiology of value-based decision making. Nature Reviews. Neuroscience, 9(7), 545-556. https://doi.org/10.1038/nrn2357

Redlich, R., Almeida, J. J. R., Grotegerd, D., Opel, N., Kugel, H., Heindel, W., ... Dannlowski, U. (2014). Brain morphometric biomarkers distinguishing unipolar and bipolar depression. A voxel-based morphometry-pattern classification approach. JAMA Psychiatry, 71(11), 1222-1230. https://doi.org/10.1001/jamapsychiatry.2014.1100

Redlich, R., Dohm, K., Grotegerd, D., Opel, N., Zwitserlood, P., Heindel, W., ... Dannlowski, U. (2015). Reward processing in unipolar and bipolar depression: A functional MRI study. Neuropsychopharmacology, 40(11), 2623-2631. https://doi.org/10.1038/npp.2015.110

Reich, W., Herjanic, B., Welner, Z., \& Gandhy, P. R. (1982). Development of a structured psychiatric Interview for children: Agreement on diagnosis comparing child and parent interviews. Journal of Abnormal Child Psychology, 10(3), 325-336. https://doi.org/10.1007/BF00912325

Remijnse, P. L., Nielen, M. M. A., van Balkom, A. J. L. M., Hendriks, G.-J., Hoogendijk, W. J., Uylings, H. B. M., \& Veltman, D. J. (2009). Differential frontal-striatal and paralimbic activity during reversal learning in major depressive disorder and obsessive-compulsive disorder. Psychological Medicine, 39(9), 1503-1518. https://doi.org/10.1017/S0033291708005072

Rice, J. P., Reich, T., Bucholz, K. K., Neuman, R. J., Fishman, R., Rochberg, N., ... Begleiter, H. (1995). Comparison of direct interview and family history diagnoses of alcohol dependence. Alcoholism, Clinical and Experimental Research, 19(4), 1018-1023.

Rive, M. M., van Rooijen, G., Veltman, D. J., Phillips, M. L., Schene, A. H., \& Ruhé, H. G. (2013). Neural correlates of dysfunctional emotion regulation in major depressive disorder. A systematic review of neuroimaging studies.

Neuroscience and Biobehavioral Reviews, 37(10 Pt 2), 2529-2553. https://doi.org/10.1016/j.neubiorev.2013.07.018

Rizvi, S. J., Salomons, T. V., Konarski, J. Z., Downar, J., Giacobbe, P., McIntyre, R. S., \& Kennedy, S. H. (2013). Neural response to emotional stimuli associated with successful antidepressant treatment and behavioral activation. Journal of Affective Disorders, 151(2), 573-581. https://doi.org/10.1016/j.jad.2013.06.050

Robbins, T. W. (2016). Illuminating anhedonia. Science, 351(6268), 24-25. https://doi.org/10.1126/science.aad9698

Roiser, J. P., Elliott, R., \& Sahakian, B. J. (2012). Cognitive mechanisms of treatment in depression. Neuropsychopharmacology, 37(1), 117-136. https://doi.org/10.1038/npp.2011.183

Rosenblau, G., Sterzer, P., Stoy, M., Park, S., Friedel, E., Heinz, A., ... Ströhle, A. (2012). Functional neuroanatomy of emotion processing in major depressive disorder is altered after successful antidepressant therapy. Journal of Psychopharmacology, 26(11), 1424-1433. https://doi.org/10.1177/0269881112450779

Russo, S. J., \& Nestler, E. J. (2013). The brain reward circuitry in mood disorders. Nature Reviews. Neuroscience, 14(9). https://doi.org/10.1038/nrn3381

Rutter, M. (2011). Research Review: Child psychiatric diagnosis and classification: concepts, findings, challenges and potential. Journal of Child Psychology and Psychiatry, 52(6), 647-660. https://doi.org/10.1111/j.1469-7610.2011.02367.x 
Saez, R. A., Saez, A., Paton, J. J., Lau, B., \& Salzman, C. D. (2017). Distinct roles for the amygdala and orbitofrontal cortex in representing the relative amount of expected reward. Neuron, 95(1), 70-77.e3. https://doi.org/10.1016/j.neuron.2017.06.012

Satterthwaite, T. D., Kable, J. W., Vandekar, L., Katchmar, N., Bassett, D. S., Baldassano, C. F., ... Wolf, D. H. (2015). Common and dissociable dysfunction of the reward system in bipolar and unipolar depression. Neuropsychopharmacology, 40(9), 2258-2268. https://doi.org/10.1038/npp.2015.75

Scheuerecker, J., Meisenzahl, E. M., Koutsouleris, N., Roesner, M., Schöpf, V., Linn, J., ... Frodl, T. (2010). Orbitofrontal volume reductions during emotion recognition in patients with major depression. Journal of Psychiatry \& Neuroscience: JPN, 35(5), 311-320. https://doi.org/10.1503/jpn.090076

Schiller, C. E., Minkel, J., Smoski, M. J., \& Dichter, G. S. (2013). Remitted major depression is characterized by reduced prefrontal cortex reactivity to reward loss. Journal of Affective Disorders, 151(2), 756-762. https://doi.org/10.1016/j.jad.2013.06.016

Schmidt, L., Cléry-Melin, M.-L., Lafargue, G., Valabrègue, R., Fossati, P., Dubois, B., \& Pessiglione, M. (2009). Get aroused and be stronger: Emotional facilitation of physical effort in the human brain. Journal of Neuroscience, 29(30), 9450-9457. https://doi.org/10.1523/JNEUROSCI.1951-09.2009

Sedgwick, P. (2014). Case-control studies: advantages and disadvantages. BMJ, 348, f7707. https://doi.org/10.1136/bmj.f7707

Segarra, N., Metastasio, A., Ziauddeen, H., Spencer, J., Reinders, N. R., Dudas, R. B., ... Murray, G. K. (2016). Abnormal frontostriatal activity during unexpected reward receipt in depression and schizophrenia: Relationship to anhedonia. Neuropsychopharmacology, 41(8), 2001-2010. https://doi.org/10.1038/npp.2015.370

Sharma, A., Satterthwaite, T. D., Vandekar, L., Katchmar, N., Daldal, A., Ruparel, K., ... Wolf, D. H. (2016). Divergent relationship of depression severity to social reward responses among patients with bipolar versus unipolar depression. Psychiatry Research: Neuroimaging, 254, 18-25. https://doi.org/10.1016/j.pscychresns.2016.06.003

Sharp, C., Kim, S., Herman, L., Pane, H., Reuter, T., \& Strathearn, L. (2014). Major depression in mothers predicts reduced ventral striatum activation in adolescent female offspring with and without depression. Journal of Abnormal Psychology, 123(2), 298-309. https://doi.org/10.1037/a0036191

Sharpe, M. J., Stalnaker, T., Schuck, N. W., Killcross, S., Schoenbaum, G., \& Niv, Y. (2019). An integrated model of action selection: Distinct modes of cortical control of striatal decision making. Annual Review of Psychology, 70, 53-76. https://doi.org/10.1146/annurev-psych-010418-102824

Singh, M. K., Kelley, R. G., Howe, M. E., Reiss, A. L., Gotlib, I. H., \& Chang, K. D. (2014). Reward processing in healthy offspring of parents with bipolar disorder. JAMA Psychiatry. https://doi.org/10.1001/jamapsychiatry.2014.1031

Smith, D. V., Gseir, M., Speer, M. E., \& Delgado, M. R. (2016). Toward a cumulative science of functional integration: A meta-analysis of psychophysiological interactions. Human Brain Mapping, 37(8), 2904-2917. https://doi.org/10.1002/hbm.23216 
Smith, D. V., Rigney, A. E., \& Delgado, M. R. (2016). Distinct reward properties are encoded via corticostriatal interactions. Scientific Reports, 6, 20093. https://doi.org/10.1038/srep20093

Smith, J. D. (2012). Single-case experimental designs: A systematic review of published research and current standards. Psychological Methods, 17(4), 510 550. https://doi.org/10.1037/a0029312

Smoski, M. J., Felder, J., Bizzell, J., Green, S. R., Ernst, M., Lynch, T. R., \& Dichter, G. S. (2009). FMRI of alterations in reward selection, anticipation, and feedback in major depressive disorder. Journal of Affective Disorders, 118(13), 69-78. https://doi.org/10.1016/j.jad.2009.01.034

Smoski, M. J., Rittenberg, A., \& Dichter, G. S. (2011). Major depressive disorder is characterized by greater reward network activation to monetary than pleasant image rewards. Psychiatry Research, 194(3), 263-270.

https://doi.org/10.1016/j.pscychresns.2011.06.012

Somerville, L. H., Jones, R. M., \& Casey, B. (2010). A time of change: Behavioral and neural correlates of adolescent sensitivity to appetitive and aversive environmental cues. Brain and Cognition, 72(1), 124. https://doi.org/10.1016/j.bandc.2009.07.003

Stalnaker, T. A., Cooch, N. K., \& Schoenbaum, G. (2015). What the orbitofrontal cortex does not do. Nature Neuroscience, 18(5), 620-627. https://doi.org/10.1038/nn.3982

Steinberg, L. (2007). Risk taking in adolescence: New perspectives from brain and behavioral science. Current Directions in Psychological Science, 16(2), 5559. https://doi.org/10.1111/j.1467-8721.2007.00475.x

Steinberg, L. (2008). A social neuroscience perspective on adolescent risk-taking. Developmental Review : DR, 28(1), 78-106. https://doi.org/10.1016/j.dr.2007.08.002

Steinberg, L. (2010). A dual systems model of adolescent risk-taking. Developmental Psychobiology, 52(3), 216-224. https://doi.org/10.1002/dev.20445

Steinberg, L., Albert, D., Cauffman, E., Banich, M., Graham, S., \& Woolard, J. (2008). Age differences in sensation seeking and impulsivity as indexed by behavior and self-report: Evidence for a dual systems model. Developmental Psychology, 44(6), 1764-1778. https://doi.org/10.1037/a0012955

Steinberg, L., Graham, S., O’Brien, L., Woolard, J., Cauffman, E., \& Banich, M. (2009). Age differences in future orientation and delay discounting. Child Development, 80(1), 28-44. https://doi.org/10.1111/j.1467-8624.2008.01244.x

Steinberg, L., Icenogle, G., Shulman, E. P., Breiner, K., Chein, J., Bacchini, D., ... Takash, H. M. S. (2018). Around the world, adolescence is a time of heightened sensation seeking and immature self-regulation. Developmental Science, 21(2), e12532. https://doi.org/10.1111/desc. 12532

Stringaris, A., Vidal-Ribas Belil, P., Artiges, E., Lemaitre, H., Gollier-Briant, F., Wolke, S., ... IMAGEN Consortium. (2015). The brain's response to reward anticipation and depression in adolescence: Dimensionality, specificity, and longitudinal predictions in a community-based sample. The American Journal of Psychiatry, 172(12), 1215-1223. https://doi.org/10.1176/appi.ajp.2015.14101298

Surguladze, S. A., Brammer, M. J., Keedwell, P., Giampietro, V., Young, A. W., Travis, M. J., ... Phillips, M. L. (2005). A differential pattern of neural response toward sad versus happy facial expressions in major depressive 
disorder. Biological Psychiatry, 57(3), 201-209.

https://doi.org/10.1016/j.biopsych.2004.10.028

Surguladze, S. A., El-Hage, W., Dalgleish, T., Radua, J., Gohier, B., \& Phillips, M. L. (2010). Depression is associated with increased sensitivity to signals of disgust: A functional magnetic resonance imaging study. Journal of Psychiatric Research, 44(14), 894-902. https://doi.org/10.1016/j.jpsychires.2010.02.010

Swartz, J. R., Knodt, A. R., Radtke, S. R., \& Hariri, A. R. (2015). A neural biomarker of psychological vulnerability to future life stress. Neuron, 85(3), 505-511. https://doi.org/10.1016/j.neuron.2014.12.055

Townsend, J. D., Eberhart, N. K., Bookheimer, S. Y., Eisenberger, N. I., Foland-Ross, L. C., Cook, I. A., ... Altshuler, L. L. (2010). fMRI activation in amygdala and orbitofrontal cortex in unmedicated subjects with major depressive disorder. Psychiatry Research, 183(3), 209-217. https://doi.org/10.1016/j.pscychresns.2010.06.001

Tremblay, L. K., Naranjo, C. A., Cardenas, L., Herrmann, N., \& Busto, U. E. (2002). Probing brain reward system function in major depressive disorder: Altered response to dextroamphetamine. Archives of General Psychiatry, 59(5), 409416. https://doi.org/10.1001/archpsyc.59.5.409

Tremblay, L. K., Naranjo, C. A., Graham, S. J., Herrmann, N., Mayberg, H. S., Hevenor, S., \& Busto, U. E. (2005). Functional neuroanatomical substrates of altered reward processing in major depressive disorder revealed by a dopaminergic probe. Archives of General Psychiatry, 62(11), 1228-1236. https://doi.org/10.1001/archpsyc.62.11.1228

Triantafyllou, C., Hoge, R. D., Krueger, G., Wiggins, C. J., Potthast, A., Wiggins, G. C., \& Wald, L. L. (2005). Comparison of physiological noise at 1.5 T, $3 \mathrm{~T}$ and $7 \mathrm{~T}$ and optimization of fMRI acquisition parameters. NeuroImage, 26(1), 243-250. https://doi.org/10.1016/j.neuroimage.2005.01.007

Tsukiura, T., \& Cabeza, R. (2008). Orbitofrontal and hippocampal contributions to memory for face-name associations: The rewarding power of a smile. Neuropsychologia, 46(9), 2310-2319. https://doi.org/10.1016/j.neuropsychologia.2008.03.013

Turkeltaub, P. E., Eickhoff, S. B., Laird, A. R., Fox, M., Wiener, M., \& Fox, P. (2012). Minimizing within-experiment and within-group effects in Activation Likelihood Estimation meta-analyses. Human Brain Mapping, 33(1), 1-13. https://doi.org/10.1002/hbm.21186

Tustison, N. J., Avants, B. B., Cook, P. A., Yuanjie Zheng, Egan, A., Yushkevich, P. A., \& Gee, J. C. (2010). N4ITK: Improved N3 Bias Correction. IEEE Transactions on Medical Imaging, 29(6), 1310-1320. https://doi.org/10.1109/TMI.2010.2046908

Uban, K. A., Horton, M. K., Jacobus, J., Heyser, C., Thompson, W. K., Tapert, S. F., ... Sowell, E. R. (2018). Biospecimens and the ABCD study: Rationale, methods of collection, measurement and early data. Developmental Cognitive Neuroscience. https://doi.org/10.1016/j.dcn.2018.03.005

Urošević, S., Collins, P., Muetzel, R., Lim, K., \& Luciana, M. (2012). Longitudinal changes in behavioral approach system sensitivity and brain structures involved in reward processing during adolescence. Developmental Psychology, 48(5), 1488-1500. https://doi.org/10.1037/a0027502 
Van Essen, D. C., Smith, S. M., Barch, D. M., Behrens, T. E. J., Yacoub, E., \& Ugurbil, K. (2013). The WU-Minn Human Connectome Project: An overview. NeuroImage, 80, 62-79. https://doi.org/10.1016/j.neuroimage.2013.05.041

Van Roy, B., Groholt, B., Heyerdahl, S., \& Clench-Aas, J. (2010). Understanding discrepancies in parent-child reporting of emotional and behavioural problems: Effects of relational and socio-demographic factors. $B M C$ Psychiatry, 10, 56. https://doi.org/10.1186/1471-244X-10-56

Veale, J. F. (2014). Edinburgh Handedness Inventory - Short Form: A revised version based on confirmatory factor analysis. Laterality: Asymmetries of Body, Brain and Cognition, 19(2), 164-177. https://doi.org/10.1080/1357650X.2013.783045

Volkow, N. D., Koob, G. F., Croyle, R. T., Bianchi, D. W., Gordon, J. A., Koroshetz, W. J., ... Weiss, S. R. B. (2017). The conception of the ABCD study: From substance use to a broad NIH collaboration. Developmental Cognitive Neuroscience. https://doi.org/10.1016/j.den.2017.10.002

Voorn, P., Vanderschuren, L. J. M. J., Groenewegen, H. J., Robbins, T. W., \& Pennartz, C. M. A. (2004). Putting a spin on the dorsal-ventral divide of the striatum. Trends in Neurosciences, 27(8), 468-474. https://doi.org/10.1016/j.tins.2004.06.006

Wagner, G., Schachtzabel, C., Peikert, G., \& Bär, K.-J. (2015). The neural basis of the abnormal self-referential processing and its impact on cognitive control in depressed patients. Human Brain Mapping, 36(7), 2781-2794. https://doi.org/10.1002/hbm.22807

Wang, K. S., Smith, D. V., \& Delgado, M. R. (2016). Using fMRI to study reward processing in humans: Past, present, and future. Journal of Neurophysiology, 115(3), 1664-1678. https://doi.org/10.1152/jn.00333.2015

Wang, L., Krishnan, K. R., Steffens, D. C., Potter, G. G., Dolcos, F., \& McCarthy, G. (2008). Depressive state- and disease-related alterations in neural responses to affective and executive challenges in geriatric depression. The American Journal of Psychiatry, 165(7), 863-871. https://doi.org/10.1176/appi.ajp.2008.07101590

Wehr, T. A., \& Goodwin, F. K. (1987). Can antidepressants cause mania and worsen the course of affective illness? The American Journal of Psychiatry, 144(11), $1403-1411$.

Weissman, M. M., Wickramaratne, P., Warner, V., John, K., Prusoff, B. A., Merikangas, K. R., \& Gammon, G. D. (1987). Assessing psychiatric disorders in children: Discrepancies between mothers' and children's reports. Archives of General Psychiatry, 44(8), 747-753. https://doi.org/10.1001/archpsyc.1987.01800200075011

Weller, E. B., Weller, R. A., \& Fristad, M. A. (1995). Bipolar disorder in children misdiagnosis, underdiagnosis, and future directions. Journal of the American Academy of Child \& Adolescent Psychiatry, 34(6), 709-714. https://doi.org/10.1097/00004583-199506000-00010

Whitton, A. E., Treadway, M. T., \& Pizzagalli, D. A. (2015). Reward processing dysfunction in major depression, bipolar disorder and schizophrenia. Current Opinion in Psychiatry, 28(1), 7-12. https://doi.org/10.1097/YCO.0000000000000122

Wimmer, G. E., Daw, N. D., \& Shohamy, D. (2012). Generalization of value in reinforcement learning by humans. The European Journal of Neuroscience, 35(7), 1092-1104. https://doi.org/10.1111/j.1460-9568.2012.08017.x 
Wimmer, G. E., \& Shohamy, D. (2012). Preference by association: How memory mechanisms in the hippocampus bias decisions. Science, 338(6104), 270-273. https://doi.org/10.1126/science.1223252

Wolf, D. H., Satterthwaite, T. D., Kantrowitz, J. J., Katchmar, N., Vandekar, L., Elliott, M. A., \& Ruparel, K. (2014). Amotivation in schizophrenia: Integrated assessment with behavioral, clinical, and imaging measures. Schizophrenia Bulletin, 40(6), 1328-1337. https://doi.org/10.1093/schbul/sbu026

Woo, C. W., Chang, L. J., Lindquist, M. A., \& Wager, T. D. (2017). Building better biomarkers: Brain models in translational neuroimaging. Nature Neuroscience, 20(3), 365-377. https://doi.org/10.1038/nn.4478

World Health Organization. (2006). World Health Report 2006: Working together for health. Geneva, Switerland.

World Health Organization. (2017). Depression and other common mental disorders: Global health estimates.

Yau, W. Y. W., Zubieta, J. K., Weiland, B. J., Samudra, P. G., Zucker, R. A., \& Heitzeg, M. M. (2012). Nucleus accumbens response to incentive stimuli anticipation in children of alcoholics: Relationships with precursive behavioral risk and lifetime alcohol use. Journal of Neuroscience, 32(7), 2544-2551. https://doi.org/10.1523/JNEUROSCI.1390-11.2012

Young, C. B., Chen, T., Nusslock, R., Keller, J., Schatzberg, A. F., \& Menon, V. (2016). Anhedonia and general distress show dissociable ventromedial prefrontal cortex connectivity in major depressive disorder. Translational Psychiatry, 6(5), e810. https://doi.org/10.1038/tp.2016.80

Young, K. D., Bodurka, J., \& Drevets, W. C. (2016). Differential neural correlates of autobiographical memory recall in bipolar and unipolar depression. Bipolar Disorders, 18(7), 571-582. https://doi.org/10.1111/bdi.12441

Youngstrom, E. A., Birmaher, B., \& Findling, R. L. (2008). Pediatric bipolar disorder: validity, phenomenology, and recommendations for diagnosis. Bipolar Disorders, 10(1 Pt 2), 194-214. https://doi.org/10.1111/j.13995618.2007.00563.x

Youngstrom, E. A., Frazier, T. W., Demeter, C., Calabrese, J. R., \& Findling, R. L. (2008). Developing a 10-Item mania scale From the Parent General Behavior Inventory for children and adolescents. The Journal of Clinical Psychiatry, 69(5), 831-839.

Zeng, L. L., Shen, H., Liu, L., Wang, L., Li, B., Fang, P., ... Hu, D. (2012). Identifying major depression using whole-brain functional connectivity: A multivariate pattern analysis. Brain, 135(5), 1498-1507. https://doi.org/10.1093/brain/aws059

Zhang, B., Li, S., Zhuo, C., Li, M., Safron, A., Genz, A., ... Walter, M. (2017). Altered task-specific deactivation in the default mode network depends on valence in patients with major depressive disorder. Journal of Affective Disorders, 207, 377-383. https://doi.org/10.1016/j.jad.2016.08.042

Zhang, W. N., Chang, S. H., Guo, L. Y., Zhang, K. L., \& Wang, J. (2013). The neural correlates of reward-related processing in major depressive disorder: A metaanalysis of functional magnetic resonance imaging studies. Journal of Affective Disorders, 151(2), 531-539. https://doi.org/10.1016/j.jad.2013.06.039

Zhang, Y., Brady, M., \& Smith, S. (2001). Segmentation of brain MR images through a hidden Markov random field model and the expectation-maximization 
algorithm. IEEE Transactions on Medical Imaging, 20(1), 45-57. https://doi.org/10.1109/42.906424

Zhong, M., Wang, X., Xiao, J., Yi, J., Zhu, X., Liao, J., ... Yao, S. (2011). Amygdala hyperactivation and prefrontal hypoactivation in subjects with cognitive vulnerability to depression. Biological Psychology, 88(2-3), 233-242. https://doi.org/10.1016/j.biopsycho.2011.08.007

Zink, C. F., Pagnoni, G., Chappelow, J., Martin-Skurski, M., \& Berns, G. S. (2006). Human striatal activation reflects degree of stimulus saliency. NeuroImage, 29(3), 977-983. https://doi.org/10.1016/j.neuroimage.2005.08.006

Zisook, S., Lesser, I., Stewart, J. W., Wisniewski, S. R., Balasubramani, G. k., Fava, M., ... Rush, A. J. (2007). Effect of age at onset on the course of major depressive disorder. American Journal of Psychiatry, 164(10), 1539-1546. https://doi.org/10.1176/appi.ajp.2007.06101757 